\title{
THE
}

8-1-1981

\section{Quantum Spin Dynamics of the Antiferromagnetic Linear Chain in Zero and Nonzero Magnetic Field}

Gerhard Müller

University of Rhode Island, gmuller@uri.edu

Harry Thomas

Hans Beck

Jill C. Bonner

University of Rhode Island

Follow this and additional works at: https://digitalcommons.uri.edu/phys_facpubs

Terms of Use

All rights reserved under copyright.

\section{Citation/Publisher Attribution}

Müller, G., Thomas, H., Beck, H., \& Bonner, J. C. (1981). Quantum spin dynamics of the antiferromagnetic linear chain in zero and nonzero magnetic field. Phys. Rev. B 24(3), 1429-1467. doi: 10.1103/ PhysRevB.24.1429.

Available at: http://dx.doi.org/10.1103/PhysRevB.24.1429.

This Article is brought to you for free and open access by the Physics at DigitalCommons@URI. It has been accepted for inclusion in Physics Faculty Publications by an authorized administrator of DigitalCommons@URI. For more information, please contact digitalcommons-group@uri.edu. 


\title{
Quantum spin dynamics of the antiferromagnetic linear chain in zero and nonzero magnetic field
}

\author{
Gerhard Müller and Harry Thomas \\ Institut für Physik der Universität Basel, CH-4056 Basel, Switzerland \\ Hans Beck \\ Institut de Physique de I'Université de Neuchâtel, CH-2000 Neuchâtel, Switzerland \\ Jill C. Bonner \\ Department of Physics, University of Rhode Island. Kingston. Rhode Island 0288I
}

(Received 19 August 1980)

\begin{abstract}
Spin-dynamical calculations on one-dimensional systems have relied heavily on classical $(s=\infty)$ theories, despite abundant evidence that quantum effects can be extremely important at low temperatures. We present a new approach to the spin dynamics of the one-dimensional isotropic $s=\frac{1}{2}$ Heisenberg antiferromagnetic (HB AF) which does not involve the many-body techniques usually employed. It is based on analytic Bethe ansatz calculations of excitation energies and densities of states combined with finite-chain calculations of matrix elements. An important feature of our method is the use of rigorous selection rules and the introduction of new selection rules, which are valid for macroscopic systems in a magnetic field. We show that in . zero field the dynamical two-spin correlation function $S_{\mu \mu}(q, \omega)$ at $T=0$ is governed by a twoparameter continuum of spin-wave-type excitations. In nonzero field, the longitudinal component $S_{z z}(q, \omega)$ and the transverse components $S_{x x}(q, \omega) \equiv S_{v v}(q, \omega)$ behave quite differently because they are dominated by different continua of excitations. The former is characterized by a lowest excitation branch with a zero-frequency mode moving from the zone boundary $(q=\pi)$ towards the zone center $(q=0)$ as the field increases, whereas the latter is characterized by a lowest branch with a zero frequency mode moving from $q=0$ to $\pi$ with increasing field. The first part of our work features an approximate analytic expression for $S_{\mu \mu}(q, \omega)$ at zero temperature and in zero field. Although our expression is not rigorous, exact sum rules are violated only by a small amount, and good agreement exists with the few known exact results. Our studies are extended to nonzero temperatures by placing major reliance on exact finite-chain calculations. Our work was stimulated by recent neutron scattering experiments and is oriented towards experimental comparisons. Our result for the $s=\frac{1}{2}$ integrated intensity is in much better agreement with neutron scattering data on $\mathrm{CuCl}_{2} \cdot 2 \mathrm{~N}\left(\mathrm{C}_{5} \mathrm{D}_{5}\right)(\mathrm{CPC})$ than the corresponding semiclassical result. Moreover, the spectral-weight distribution in $S_{\mu \mu}(q, \omega)$ shows increasing asymmetry as $q \rightarrow \pi$, a quantum effect, again in agreement with more recent neutron scattering data. The second part of our work deals with the effects of an applied magnetic field. We extend the analytic work of Ishimura and Shiba to obtain expressions for the energies and densities of states of the various excitation continua. It is shown that these continua are expected to give rise to multiple structures in the scattering intensity. Our results appear to be in quantitative agreement with preliminary results of a neutron study in CPC in a field of $70 \mathrm{kOe}$, revealing anomalous scattering intensity peaks. Our results repeatedly demonstrate the inadequacy of classical spin-wave theory for this problem. They call for additional experimental studies on quasi-one-dimensional antiferromagnets to examine other unusual and interesting phenomena predicted by our approach.
\end{abstract}

\section{DYNAMICS IN ZERO MAGNETIC FIELD}

\section{A. Introductory survey}

Linear spin-wave theory applied to the onedimensional (1D) Heisenberg antiferromagnet
(HB AF) assumes that the system in its ground state has staggered long-range order along the chains.' This (semiclassical) theory is asserted to be valid to order $1 / s$ and therefore expected to be a good approximation for real systems with large spin quantum numbers $s$. It takes into account small deviations 
from the aligned Néel state, and the single spin waves are transverse $(\perp Z)$ excitations obeying the well-known double-sine dispersion relation of Anderson

$$
\epsilon^{\mathrm{CL}}(q)=2 J_{S}|\sin q|
$$

[ $J$ is the exchange constant of the Hamiltonian (1.4)]. The contribution of these spin waves to the low-temperature dynamics is reflected in a $\delta$ peak at the spin-wave frequency in the transverse structure function [defined in Eq. (1.8)] $S_{x x}(q, \omega)=S_{y y}(q, \omega)$ whereas the longitudinal component $S_{z z}(q, \omega)$ is predicted to exhibit a logarithmic singularity at the single spin-wave position. ${ }^{2}$ Inelastic neutron scattering studies on TMMC (tetramethyl ammonium manganese trichloride) by Hutchings et al. ${ }^{2}$ were in good agreement with Anderson's theory, and this was attributed to the fact that the effective spin $s=\frac{5}{2}$ of the manganese ion was sufficiently close to the classical limit $s=\infty$. Another way of stating the classical result is to say that the Anderson spin-wave states exhaust the sum rules for $T=0$, which implies that no higher excited states can contribute to the spin dynamics. The limitations of linear spin-wave theory are, however, intrinsically evident by a basic result: Due to the zero-point fluctuations the sublattice magnetization of the assumed Néel ground state is reduced by a certain amount. In $1 \mathrm{D}$, where the fluctuations are strongest, this spin reduction diverges when calculated in a spin-wave approximation. Thus, in 1D the symmetry-breaking Néel state is not a good approximation to the AF ground state. In fact, it has been proved that the ground state of the HB AF (for arbitrary $s$ ) must be a singlet, i.e., a state which is rotationally invariant in spin space. ${ }^{3}$

Following the work on TMMC, speculation began on the possible role of quantum effects in 1D AF spin dynamics. For the $s=\frac{1}{2} \mathrm{HB}$ AF (extreme quantum limit) the lowest excited states are the famous des Cloizeaux-Pearson (dCP) triplets (having total spin $\left.s^{T}=1\right),{ }^{4}$ which exhibit the same doublesine dispersion curve as the Anderson $s=\infty$ states (1.1), but with amplitude increased by a factor $\pi / 2$. Finite-chain calculations $\mathrm{s}^{5.6}$ as well as exact calculations by Yamada $^{7,8}$ using the Bethe ansatz approach ${ }^{9}$ revealed the existence of an extended (two-parameter) continuum of excited triplet states whose lower boundary in $(q, \omega)$ space is the $\mathrm{dCP}$ expression

$$
\epsilon_{1}(q)=\frac{\pi}{2} J|\sin q|
$$

whereas its upper boundary is given by

$$
\epsilon_{2}(q)=\pi J|\sin q / 2| \text {. }
$$

It has been shown, however, that the low-lying HB AF spectral excitations include a second double continuum of singlet $\left(s^{T}=0\right)$ states, which, though slightly displaced upwards from the triplet continuum for finite $N$, can be shown to become degenerate with the triplet continuum in the thermodynamic limit $N \rightarrow \infty{ }^{6}$ The lowest-lying excitations therefore become fourfold degenerate, in agreement with analytic work of Johnson and McCoy. ${ }^{10}$ Furthermore, as is implicit in the results of Sec. II, there are double continua of excitations with total $\operatorname{spin} s^{T}=2,3,4, \ldots$, which will, in the thermodynamic limit, also completely overlap with the triplet continuum as long as $s^{T} / N \rightarrow 0 .^{11}$

Interest, therefore, arose as to whether excitations higher in energy than the dCP spin-wave branch would contribute to the spin dynamics for the $s=\frac{1}{2}$ HB AF chain. Bonner et al., ${ }^{6}$ on the basis of numerical calculations on a system of $N=8$ spins, showed that higher-lying states in the triplet double continuum contributed a considerable amount to the spectral-weight function $S_{\mu \mu}(q, \omega)$ for $q$ values close to $\pi$, and conjectured that, in the limit $N \rightarrow \infty$, an appreciable "tail" of spectral weight should extend to the upper boundary. Hohenberg and Brinkman ${ }^{12}$ used exact sum-rule calculations in order to answer this question. However, they were only able to show that for small $q S_{\mu \mu}(q, \omega)$ should have most of its weight concentrated near the lower threshold, which is the $\mathrm{dCP}$ energy.

Calculations on the 1D $X Y$ model, which behaves very similarly to its $\mathrm{HB}$ counterpart but is far more amenable to exact calculations, have provided valuable insight into 1D spectral-weight distribution at low temperatures. A comprehensive paper by Niemeijer ${ }^{13}$ on the dynamics of the $s=\frac{1}{2} X Y$ chain has been of considerable value to us in formulating the approach to the Heisenberg spin dynamics contained in this paper. The Neimeijer work, however, is highly mathematical in approach, without discussion or evaluation of important expressions. This may explain why, in our opinion, this important paper has not so far received the attention it deserves. More explicit calculations of various dynamic quantities for the $X Y$ model at $T=0$ and finite temperatures became available shortly after the work of Niemeijer. ${ }^{14.15}$ Using the general expressions of Niemeijer it is straightforward to calculate the $T=0$ longitudinal structure function $S_{z z}(q, \omega)$ exactly. [For the result see Eq. (1.35).] It is entirely governed by a two-parameter continuum of excitations with lower and upper boundaries

$$
\begin{aligned}
& \epsilon_{1}^{x v}(q)=J|\sin q|, \\
& \epsilon_{2}^{x y}(q)=2 J|\sin q / 2| .
\end{aligned}
$$

The spectral weight is finite at the lower boundary [Eq. (1.3a)], and it increases towards the upper boundary [Eq. (1.3b)], where it has a divergence. Thus, the lower branch, which is often called specifically "the spin-wave branch" does not dominate the low- 
temperature dynamics at all in this case. This striking result shows immediately the importance of studying the higher excitations of the HB AF counterpart.

Returning to the HB AF, some experimental evidence for an asymmetrical line shape was apparent in neutron scattering studies by Endoh et al. ${ }^{16}$ on the quasi-1D $s=\frac{1}{2} \mathrm{HB} \mathrm{AF}$ compound $\mathrm{CuCl}_{2} \cdot 2 \mathrm{~N}\left(\mathrm{C}_{5} \mathrm{D}_{5}\right)$ (CPC). Such an asymmetry would be consistent with a tail of spectral weight above a low-frequency cutoff with dominating spectral weight, and would be inconsistent with classical spin-wave theory. At about the same time, some theoretical evidence for the existence of an asymmetry appeared in the work of Todani and Kawasaki. ${ }^{17}$ This work is a dynamical Hartree-Fock calculation, which reproduces the static properties of the Bulaevskii Hartree-Fock theory ${ }^{18}$ in the appropriate limit. The results, however, display nonphysical features such as a gap in the dispersion spectrum at $q=\pi$, as well as divergences in the response function at finite temperatures. The authors did not specifically relate their work to the underlying spectral excitations. A HolsteinPrimakoff-type calculation by Mikeska, ${ }^{19}$ which should be valid to order $1 / \mathrm{s}$, yielded analytic expressions for spin-correlation functions at low temperatures for $q \simeq \pi$. The Mikeska scattering function exhibits a strong asymmetry for all $s$. However, on the basis of his results, Mikeska conjectured that asymmetry effects would only be experimentally observable for $s=\frac{1}{2}$, where, of course, a semiclassical calculation is least reliable.

In the remaining subsections of Sec. II we present a fully quantum-mechanical study of the zerotemperature spin dynamics: We use (exact) finite chain calculations and apply selection rules (which will be proven) in order to identify that class of states which is relevant for the $T=0$ dynamics. We then calculate exactly in the Bethe ansatz approach energies and density of states of these relevant excitations. Finally, we use again finite chain calculations (including extrapolations) in order to find an expression for their spectral weight. We end up with an analytical expression for $S_{\mu \mu}(q, \omega)$ which, though approximate, is in good agreement with various sum rules as well as with exact calculations in the Luttinger model. Finite temperature effects are discussed and comparison with low- $T$ neutron scattering experiments is highly encouraging for peak frequency and line shape as well as integrated intensity.

\section{B. Calculational details and selection rules}

The Hamiltonian for the 1D HB AF is given by

$$
H=J \sum_{l=1}^{N} \overrightarrow{\mathbf{s}}_{l} \cdot \overrightarrow{\mathbf{s}}_{l+1}-g \mu_{B} B \sum_{l=1}^{N} s_{l}^{z} \text {. }
$$

The first term describes an isotropic AF $(J>0)$ exchange interaction between nearest-neighbor spins $\overrightarrow{\mathbf{s}}_{l}$ with quantum numbers $s=\frac{1}{2}$. The Zeeman term couples the system to a uniform magnetic field $B$ in the $z$ direction. In our notation we shall use the reduced field $h=g \mu_{B} B / J$. Periodic boundary conditions $\left(\overrightarrow{\mathbf{s}}_{N+1} \equiv \overrightarrow{\mathbf{s}}_{1}\right)$ are applied. A related model to which we shall make frequent reference because it allows for exact solutions for many aspects of the dynamics problem is the $s=\frac{1}{2} 1 \mathrm{D} X Y$ model with Hamiltonian

$$
H=J \sum_{i=1}^{N}\left(s_{i}^{r} s_{i+1}^{x}+s_{i}^{y} s_{i+1}^{v}\right)-g \mu_{B} B \sum_{i=1}^{N} s_{i} .
$$

When we deal with these models we should make use of their symmetries. The following important operators commute with $H$ : (i) the $z$ component of the total spin $s_{z}$

$$
s_{z}=\sum_{i=1}^{N} s_{i}
$$

(ii) the translation operator $T$

$$
T s_{l}{ }^{\mu} T^{+}=s_{l-1}^{\mu}, \quad \mu=x, y, z,
$$

(iii) the magnitude of the total spin $\overrightarrow{\mathrm{s}}^{2}$ (for the HB AF only)

$$
\overrightarrow{\mathrm{s}}^{2}=\left(\sum_{i=1}^{N} s_{i}^{x}\right)^{2}+\left(\sum_{i=1}^{N} s_{i}^{y^{\prime}}\right)^{2}+\left(\sum_{i=1}^{N} s_{i}\right)^{2} .
$$

All eigenstates $|\lambda\rangle$ of $H$ can therefore be characterized by useful quantum numbers:

(i)

$$
s_{z}\left|s_{z}^{T} \cdots\right\rangle=s_{z}^{T}\left|s_{z}^{T} \cdots\right\rangle,
$$

where

(ii)

$$
s_{z}^{T}=N s, \quad N s-1, \ldots,-N s
$$

$$
T|k \cdots\rangle=e^{-i k}|k \cdots\rangle,
$$

where $k$, the wave number of the state can have the values

$$
k=(2 \pi / N) n, n=0,1, \ldots, N-1,
$$

(iii)

$$
\overrightarrow{\mathrm{s}}^{2}\left|s^{T} \ldots\right\rangle=s^{T}\left(s^{T}+1\right)\left|s^{T} \ldots\right\rangle
$$

with $0 \leqslant s^{T} \leqslant N s$ (for the HB AF only).

The quantity of interest for comparison with experimentally measurable quantities is the dynamic twospin correlation function in $(q, \omega)$ space $S_{\mu \nu}(q, \omega)$ (which is also called the structure function or dynamic form factor). It is the Fourier transform of the time-dependent pair-correlation function $\left\langle s_{l}^{\mu}(t) s_{,},(0)\right\rangle$. If the eigenvalues $E_{\lambda}$ and the eigenfunctions $|\lambda\rangle$ of $H$ are known, this Fourier transform can be written as 


$$
\begin{aligned}
S_{\mu \nu}(q, \omega) & =N^{-1} \sum_{l, R} e^{i q R} \int_{-\infty}^{+\infty} d t e^{i \omega t}\left\langle s_{l}^{\mu}(t) s_{l+R}^{\nu}\right\rangle \\
& =\frac{2 \pi}{Z} \sum_{\lambda \lambda^{\prime}} e^{-\beta E_{\lambda}}\left\langle\lambda\left|s^{\mu}(q)\right| \lambda^{\prime}\right\rangle\left\langle\lambda^{\prime}\left|s^{\nu}(-q)\right| \lambda\right\rangle \delta\left(\omega+E_{\lambda}-E_{\lambda^{\prime}}\right)
\end{aligned}
$$

$Z$ is the partition function and

$$
s^{\mu}(q)=N^{-1 / 2} \sum_{l} e^{-i q l} s_{l}^{\mu} .
$$

Due to the rotational symmetry in spin space, offdiagonal components of $S_{\mu \nu}$ vanish,

$$
S_{\mu \nu}(q, \omega) \equiv 0 \text { unless } \mu=\nu,
$$

and the two transverse components are identical

$$
S_{x x}(q, \omega) \equiv S_{y y}(q, \omega) .
$$

A requirement for the isotropic $\mathrm{HB}$ AF in zero field is that they are equal to the longitudinal component $S_{z z}(q, \omega)$. This is, as stated above, violated by Anderson's two sublattice theory. Instead of $S_{x x}$ and $S_{y v}$ it may be more convenient to evaluate $S_{+-}$and $S_{-+}$using the relations

$$
s^{ \pm}(q)=s^{x}(q) \pm i s^{y}(q) \text {. }
$$

Due to the reflection symmetry of the system all the wave-number-dependent properties are necessarily even functions with respect to $q$. Hence, for simplicity in the following we present results only for $q \geqslant 0$. The number of nonvanishing terms in $S_{\mu \mu}(q, \omega)$ represented by the double sum (1.8) is considerably reduced by the use of selection rules, which result from the symmetry properties of $H$ as discussed above:

(i)

$$
\begin{aligned}
& \left\langle s_{z}^{T} \cdots\left|s^{z}(q)\right| s_{z}^{T^{\prime}} \cdots\right\rangle=0 \text { unless } s_{z}^{T}=s_{z}^{T^{\prime}} \\
& \left\langle s_{z}^{T} \cdots\left|s^{ \pm}(q)\right| s_{z}^{T^{\prime}} \cdots\right\rangle=0 \text { unless } s_{z}^{T}=s_{z}^{T^{\prime}} \pm 1
\end{aligned}
$$

These rules are easily derived from the properties of the operators $s^{z}(q), s^{ \pm}(q)$

(ii)

$$
\begin{array}{r}
\left\langle k \cdots\left|s^{\mu}(q)\right| k^{\prime} \cdots\right\rangle \propto \delta_{k^{\prime}, k+q+2 \pi n}, \\
n=0, \pm 1, \pm 2, \ldots, .
\end{array}
$$

This is easily proved by making use of the translation operator,

(iii)

$$
\begin{aligned}
& \left\langle s^{T} \cdots\left|s^{\mu}(q)\right| s^{T^{\prime}} \cdots\right\rangle=0 \\
& \text { if }\left|s^{T}-s^{T^{\prime}}\right| \neq 0,1 \text { or } s^{T}=s^{T}=s^{T^{\prime}}=0 .
\end{aligned}
$$

This is a direct result of the Wigner-Eckart theorem for the case of vector operators.

These selection rules are extremely valuable especially for $T=0$, where Eq. (1.8) reduces to a single sum

$$
S_{\mu \mu}(q, \omega)=\sum_{\lambda} M_{\lambda}^{\mu} \delta\left(\omega+E_{G}-E_{\lambda}\right) .
$$

$E_{G}$ is the energy of the ground state $|G\rangle$, and the squared matrix elements $M_{\lambda}^{\mu}$ are given by

$$
M_{\lambda}^{\mu}=2 \pi\left|\left\langle G\left|s^{\mu}(q)\right| \lambda\right\rangle\right|^{2} .
$$

The selection rules tell us which classes of states have nonzero matrix elements with the ground state (whose character changes, of course, with the magnetic field), and thereby contribute to the spin dynamics. This simplifies the dynamics problem considerably. For the special case of the HB AF in zero field, Eq. $(1.13 \mathrm{c})$ implies that since the zero-field ground state is a singlet $\left(s^{T}=0\right)$, the singlet continuum mentioned above cannot make any contribution, and neither can the low-lying states with $s^{T}=2,3, \ldots$, This feature was observed in previous finite-chain calculations lacking, however, an explanation. ${ }^{6}$ The important result is that in this case the spin dynamics are completely determined by the triplet $\left(s^{T}=1\right)$ excitations.

Figure 1 shows $S_{z z}(q, \omega)$ for a cyclic chain of $N=10$ spins in a representation which is most suitable for the $T=0$ dynamics results for small systems. We may call it the "spectral representation." For each value of the wave number $q=(2 \pi / N) n$, $n=0,1, \ldots, 5, S_{z z}(q, \omega)$ is a set of $\delta$ functions according to Eq. (1.14). The triangles denote energy and wave number of the triplet excitations, and the numbers represent the corresponding spectral weight $M_{\lambda}^{z}$. The circle indicates the singlet ground state. Figure 1 is strongly suggestive of the important point we present and emphasize in this work. The dominant contribution to the spin dynamics comes from triplet states lying between the two solid lines which 


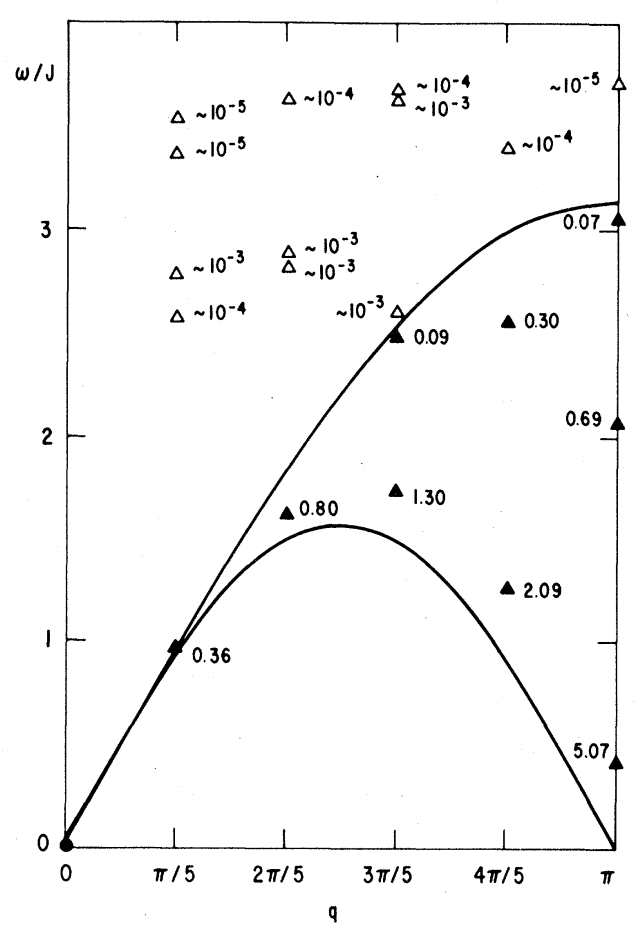

FIG. 1. Spectral representation of $S_{z z}(q, \omega)\left[\equiv S_{y v}(q, \omega)\right.$ $\left.\equiv S_{x x}(q, \omega)\right]$ at $T=0$ and $h=0$ for the $1 \mathrm{D} \mathrm{HB} \mathrm{AF}$ with $N=10$. The circle denotes the singlet $\left(s^{T}=0\right)$ ground state and the triangles triplet $\left(s^{T}=1\right)$ excitations together with their spectral weight $M_{\lambda}^{z}$. The solid lines represent the lower boundary $\epsilon_{1}(q)$ and upper boundary $\epsilon_{2}(q)$ of the SWC. The full symbols distinguish those SWC states which can be identified in the Bethe ansatz approach for finite $N$ as well as for $N \rightarrow \infty$.

correspond to the dispersion curves $\epsilon_{1}(q)$ and $\epsilon_{2}(q)$ of Eq. (1.2). In the thermodynamic limit $(N \rightarrow \infty)$ these states form a two-parameter continuum ("spin-wave continuum," SWC) in $(q, \omega)$ space with boundaries $\epsilon_{1}(q)$ and $\epsilon_{2}(q)$. This feature is indicated by our finite-chain calculations, which show with increasing $N$ more states appearing and closing up as $1 / N$ both as a function of $q$ and $\omega$. However, we will generally use $N=10$ for presentation purposes in this paper. It is important to note that there are also triplet states lying above and outside the upper continuum boundary $\epsilon_{2}(q)$. However, their spectral weight is observed to be lower by at least one order of magnitude compared with the states of the SWC. Additional triplet states with even higher energy, which are not shown in Fig. 1, have extremely small spectral weight. The question of whether any spectral weight persists in the thermodynamic limit for the "triplet sea" outside the SWC is interesting and will be considered later in this section. Finally, we observe in Fig. 1 that the matrix elements of the SWC states increase in magnitude as the energy decreases to the lower boundary $\epsilon_{1}(q)$, which affirms the importance of the dCP spin-wave branch for the dynamics.

\section{Approximate analytic expression for $S_{z z}(q, \omega)$}

Using information derived from finite-chain results, selection rules, and exact dispersion curves described above, we have postulated ${ }^{20-22}$ the following analytic, albeit approximate, expression for the dynamical correlation function in $(q, \omega)$ space

$$
\begin{aligned}
S_{z z}(q, \omega)= & \frac{A}{\left[\omega^{2}-\epsilon_{1}^{2}(q)\right]^{1 / 2}} \\
& \times \Theta\left(\omega-\epsilon_{1}(q)\right) \Theta\left(\epsilon_{2}(q)-\omega\right),
\end{aligned}
$$

where $\Theta(x)$ is the step function, and $A$ is an important constant which occurs repeatedly as we examine various aspects of the physics of this problem. This function diverges at the lower boundary

$$
\epsilon_{1}(q)=(\pi / 2) J|\sin q|,
$$

which is the $\mathrm{dCP}$ spin-wave frequency, and has a tail out to

$$
\epsilon_{2}(q)=\pi J|\sin (q / 2)|,
$$

the upper cutoff energy. The expression (1.16) is that part of the total correlation function $S_{z z}(q, \omega)$ which comes from the triplet SWC. The approximation results from the fact that triplet states from outside the SWC are ignored. We shall see later that the deviation from the exact result must be small. Figure 2 shows expression (1.16) plotted in relation to the upper and lower boundary of the SWC for various fixed $q$. For small wave numbers the two boundaries are close together in $\omega$. Hence the effects of the tail become much more pronounced as $q$ approaches the zone boundary. This feature is emphasized by finite-temperature rounding effects sketched as dashed lines. The step height $\Delta$ at the upper cutoff frequency depends on the constant $A$ :

$$
\Delta=\frac{A}{\pi J \sin ^{2} q / 2} \text {. }
$$

Note that for $0<q<\pi(1.16)$ has a square-root divergence $\left[\omega-\epsilon_{1}(q)\right]^{-1 / 2}$, whereas at $q=\pi$ the divergence is stronger. $S_{z z}(q, \omega) \sim \omega^{-1}$ reflecting a large integrated intensity at the zone boundary, as is expected for an antiferromagnet. The integral $\int d q d \omega S(q, \omega)$ of Eq. (1.16) remains nonetheless finite, as required by the sum rule (1.44) (see Sec. I E).

Here we should include a remark on the "zone boundary" in 1D AF systems, a term whose use is not unique and which has therefore led to some con- 


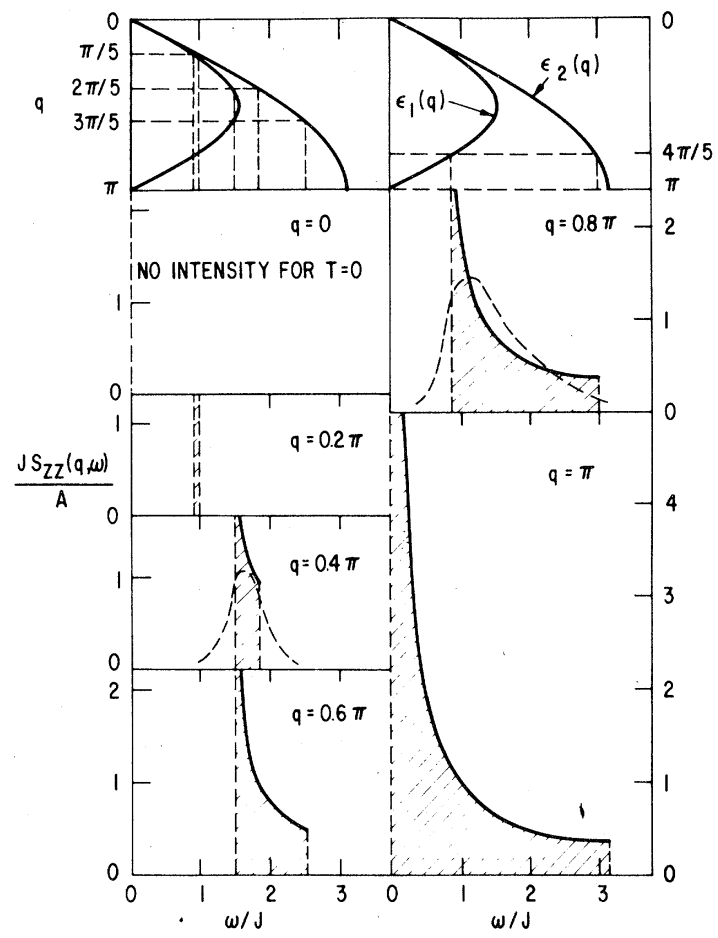

FIG. 2. Our conjecture for $S_{z z}(q, \omega)$ at $T=0$ and $h=0$ plotted as a function of $\omega$ for fixed $q=(2 \pi / 10) n$,

$n=0,1, \ldots, 5$. The top of the figure shows the boundaries of the SWC which determine the cutoffs of $S_{z z}(q, \omega)$. For $q=0$ an exact calculation predicts zero spectral weight in agreement with our conjecture. Finite temperature rounding effects are sketched as dashed lines.

fusion and even to wrong interpretations of results. The reason lies in the different translational invariance of the classical and the quantum-mechanical AF ground state. In the classical theory, where the ground state is the ordered Néel state, the magnetic unit cell is twice as large as the chemical unit cell. Hence, taking the distance between neighboring spins as unity, the Brillouin zone of the classical HB AF extends to $q=\pi / 2$. However, the quantum theory of the $1 \mathrm{D} \mathrm{HB} \mathrm{AF}$, which gives in any case a more accurate description of the corresponding real quasi-1D spin system, yields a ground state without true longrange order. This means that the magnetic unit cell is the same as the chemical one, and the Brillouin zone clearly extends out to $q=\pi$ rather than $\pi / 2$. This difference has direct consequences in quasielastic neutron scattering. Whereas the classical theory predicts a sharp Bragg peak at the "reciprocal-lattice site" $q=\pi$ due to the magnetic long-range order, the quantum theory predicts a peak with finite width at the "zone boundary" $q=\pi$, owing to the slow algebraic decay of the correlation function. As an unfortunate consequence of this different nomenclature the value of the pioneering paper by Richards and Carboni ${ }^{23}$ on finite-chain spin dynamics is handicapped by the fact that they have chosen the wrong wave number for comparing their calculations with experimental results on TMMC.

\section{Bethe ansatz calculations and matrix elements}

In this subsection we identify those excitations of the finite system (in Fig. 1 drawn as full triangles) which have dominant spectral weight, i.e., the SWC states, with a special class of eigenstates in the Bethe formalism. ${ }^{9}$ We show explicitly that in the thermodynamic limit these states form a two parameter continuum between the dispersion branches $\epsilon_{1}(q)$ and $\epsilon_{2}(q)$ of Eqs. (1.2) as described qualitatively above. This portion parallels earlier work of Yamada. ${ }^{7}$ However, we extend this work to derive new results for the density of states in the SWC and for the matrix elements between the ground state and the SWC excitations. We calculate corresponding quantities for the $X Y$ linear chain, and in Sec. II we shall show how the Bethe ansatz approach may be used to find the appropriate continuum boundaries in a nonzero magnetic field. A preliminary account of these Bethe ansatz calculations has already been presented. ${ }^{8}$

The Bethe ansatz for the exact eigenfunctions consists of a linear combination

$$
\Psi=\sum a\left(n_{1}, \ldots, n_{r}\right) \phi\left(n_{1}, \ldots, n_{r}\right)
$$

of local basis vectors with reversed spins (with respect to the ferromagnetic state) at lattice sites $n_{1}, \ldots, n_{r}$ and with coefficients of the form

$a\left(n_{1}, \ldots, n_{r}\right)=\sum_{p} \exp \left(i \sum_{j=1}^{r} k_{p_{j}} n_{j}+\frac{1}{2} i \sum_{j<1} \Psi p_{j} p_{l}\right)$,

where the summation extends over all permutations of the integers $1,2, \ldots, r$ and $p_{j}$ is the image of $j$ under the $p$ th permutation. The $k_{j}$ and the $\Psi_{i j}$ obey the coupled equations

$$
\begin{aligned}
& 2 \cot \frac{1}{2} \Psi_{j l}=\cot \frac{1}{2} k_{j}-\cot \frac{1}{2} k_{l}, \\
& N k_{j}=2 \pi \lambda_{j}+\sum_{l \neq j} \Psi_{j l} .
\end{aligned}
$$

The $\lambda_{j}$ are integers confined to $0 \leqslant \lambda_{j} \leqslant N-1$, and the choice of a set $\left\{\lambda_{j}\right\}$ determines an eigenstate of the system. Having solved Eqs. (1.20) for $k_{i}$, the wave number and the energy of the corresponding 
eigenstate can be calculated through

$$
\begin{aligned}
& k=\sum_{j=1}^{r} k_{j}=\frac{2}{N} \pi \sum_{j=1}^{r} \lambda_{j}, \\
& E=-\sum_{j=1}^{r}\left(1-\cos k_{j}\right)+E_{F},
\end{aligned}
$$

where the constant $E_{F}$ is the energy of the ferromagnetic state $E_{F}=N / 4$. The SWC excitations, in which we are interested, belong to a class of eigenstates (called "class $C$ ") identified by sets $\left\{\lambda_{j}\right\}$

$(j=1,2, \ldots, r)$ which are subject to the following restrictions ${ }^{24}$ :

$$
1 \leqslant \lambda_{j} \leqslant N-1 \text { and } \lambda_{j+1}-\lambda_{j} \geqslant 2 \text {. }
$$

The special properties of the "class $C$ " states are discussed extensively by Griffiths. ${ }^{24}$

Generally, the quantum number $s_{z}^{T}$ of a state is related to the number $r$ of $\lambda_{j}$ 's through

$$
s_{z}^{T}=\frac{N}{2}-r
$$

The quantum number $s^{T}$ of some multiplet is related to the number $r^{\prime}$ of nonzero $\lambda_{j}$ 's [satisfying Eq. (1.23)] through

$$
s^{T}=\frac{N}{2}-r^{\prime} .
$$

Actually, a set of $\lambda_{j}$ 's which are all nonzero characterizes that state of the multiplet for which $s_{z}^{T}=s^{T}$. The remaining $2 s^{T}$ components are generated by adding a number of $r-r^{\prime}\left(\leqslant 2 s^{T}\right)$ integers $\lambda_{j}=0$ to the same set of nonzero $\lambda_{j}$ 's. The energies of such components, of course, differ only in a magnetic field by Zeeman splitting. In the Bethe ansatz calculations, we will always consider the states with $s_{z}^{T}=s^{T}$, i.e., $r=r^{\prime}$. (These facts are implicit in Refs. 4 and 24.) For fixed $r$ the energy of a state is lowest when the $k_{j}$ cluster near the center of the interval $[0,2 \pi]$. In terms of the coupled equations (1.20) this implies that the $\lambda_{j}$ cluster near the center of the interval $[0, N]$, subject to the constraint (1.23).

The prescription for the AF ground state, which is a singlet $\left(s^{T}=0\right)$ for even $N$, corresponds to the set of $r=N / 2$ integers

$$
\left\{\lambda_{j}\right\}=\{1,3,5, \ldots, N-1\} .
$$

According to Eq. (1.21) the ground state has wave number $k_{G}=0$ for even $N / 2$ and $k_{G}=\pi$ for odd $N / 2$. Hulthén calculated its energy (for $N \rightarrow \infty$ ), using the above equations ${ }^{25}$ to obtain

$$
E_{G}=-N\left(\ln 2-\frac{1}{4}\right)
$$

The states belonging to the triplet $\left(s^{T}=1\right) \mathrm{SWC}$ can systematically be generated by distributing $(N / 2-1)$ integers $\lambda_{j}$ in accordance with the above rules, for a total of $N(N+2) / 8$ states. The missing $\lambda$ generally allows for a distribution of the remaining $\lambda_{j}$ 's into three clusters without violating Eq. (1.23). Each SWC state can therefore be characterized by the positions $\bar{\lambda}_{1}$ and $\bar{\lambda}_{2}$ of the two possible holes. We shall use quantum numbers $q=(2 \pi / N)\left(\bar{\lambda}_{1}+\bar{\lambda}_{2}\right)$ and $q_{m}=(2 \pi / N) \bar{\lambda}_{1}$, where $q$ (taken modulo $\left.2 \pi\right)$ denotes the total wave number (1.21) relative to that of the ground state, and $q_{m}$ denotes the different branches. Solving the coupled equations $(1.20)$ by the method of des Cloizeaux and Pearson ${ }^{4}$ or des Cloizeaux and Gaudin ${ }^{26}$ for the thermodynamic limit we obtain (for $q \geqslant 0$ and $0 \leqslant q_{m} \leqslant q$ )

$$
\omega_{m}(q)=\pi J \sin \frac{q}{2} \cos \left(\frac{q}{2}-\frac{q_{m}}{2}\right) .
$$

Details of the calculation are presented in Appendixes $A$ and $B$ for the more general case with a magnetic field present. We see at once the substitution of $q_{m}=0$ recovers the dCP spin-wave branch $\epsilon_{1}(q)$ and $q_{m}=q$ the upper boundary $\epsilon_{2}(q)$ of the SWC.

The class $C$ of states for the HB AF can be mapped into a corresponding class of states for the $X Y$ model. ${ }^{26}$ An analogous calculation leads to SWC states with energy $\left(q \geqslant 0\right.$ and $\left.0 \leqslant q_{m} \leqslant q\right)$

$$
\omega_{i m}^{x y}(q)=2 J \sin \frac{q}{2} \cos \left(\frac{q}{2}-\frac{q_{m}}{2}\right) .
$$

Again, substitution of $q_{m}=0$ and $q$ yields the lower and upper boundaries $\epsilon_{1}^{x y}(q)$ and $\epsilon_{2}^{x v}(q)$ of Eqs. (1.3). Using the formulation it is also possible to investigate analytically the finite system. The SWC states of the $X Y$ model for $N=10$ are displayed in Fig. 3. Three excitation branches are obtained, corresponding to $q_{m}=(2 \pi / 10) m, m=1,3,5$, each containing as many states as the rules (1.23) permit. Figure 3 shows their wave numbers and energies with respect to the ground state and their spectral weight $M_{\bar{\lambda}}^{z}$. Evidently, relation (1.28) is exactly obeyed by the states of a finite system using the appropriate labels $m$ and $n$. The squared matrix elements are the same for all SWC states

$$
M_{m n}^{z} \equiv 2 \pi\left|\left\langle G\left|s^{z}(q)\right| m n\right\rangle\right|^{2}=\frac{4 \pi}{N}
$$

except at the upper boundary, where $M_{m n}^{z}=(2 \pi / N)$. This difference is negligible in the thermodynamic limit. The matrix elements (1.29) can be evaluated exactly in the fermion representation. Here, the eigenstates contributing to $S_{z z}$ are identified as two particle-hole excitations with spectral weights $M_{\bar{\lambda}}^{z}=2 \pi / N$ which are degenerate except at $\epsilon_{2}^{x v}(q){ }^{27}$ This leads to the same spectral weight distribution as in Fig. 3. The apparent difference in the number of excitations is explained as follows. Since the two-fold degeneracy involves states which have all quantum numbers equal to each other due to symmetries, we are free to choose an orthogonal basis within each 


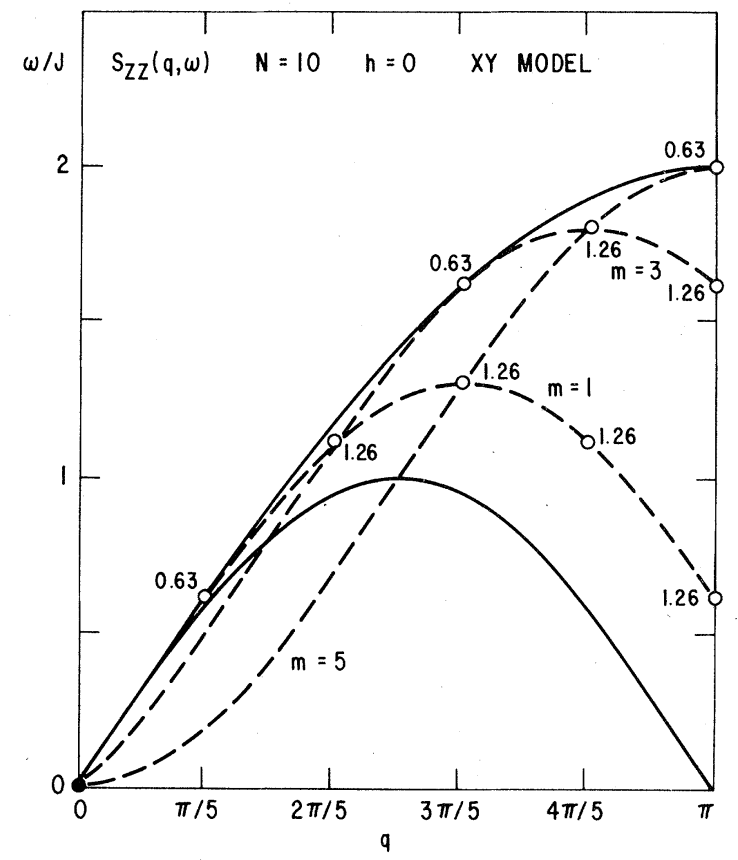

FIG. 3. Spectral representation of the longitudinal correlation function $S_{z z}(q, \omega)$ at $T=0$ and $h=0$ for the $1 \mathrm{D} X Y$ model with $N=10$. The full circle denotes the ground state and the open circles the SWC excitations together with their spectral weight $M_{\bar{\lambda}}$. The solid lines represent the lower boundary $\epsilon_{1}^{x y}(q)$ and the upper boundary $\epsilon_{2}^{x y}(q)$ of the SWC. The dashed lines give the three branches $(m=1,3,5)$.

two-dimensional subspace. In the fermion picture, we are obviously led to a basis consisting of two particle-hole excitations with equivalent spectral weight $M_{\lambda}^{z}=2 \pi / N$ in $S_{z z}(q, \omega)$. In the Bethe formalism, on the other hand, we are led to a different choice, consisting of a SWC state with spectra weight $M_{\lambda}^{z}=4 \pi / N$ and a second state with zero spectral weight which does not belong to class $C$. For completeness we should remark that in finite-chain calculations, where the Hamiltonian is diagonalized numerically, the computer provides a more or less arbitrary basis consisting of two degenerate states with spectral weight adding up to a total of $4 \pi / N$ (see Ref. 27). The SWC contribution to the dynamic correlation function, which is defined for $T=0$ according to Eq. (1.14) as

$$
S_{z z}(q, \omega)=\sum_{m, n} M_{m n} \delta\left(\omega-\omega_{m}(q)\right),
$$

can be written in the thermodynamic limit as a product

$$
S_{z z}(q, \omega) \equiv M^{z}(q, \omega) D(q, \omega) .
$$

Here

$$
M^{z}(q, \omega)=\frac{4 \pi}{N}=\mathrm{const}
$$

represents the squared matrix elements (a constant for the $X Y$ model $)$ and $D(q, \omega)$ is the density of states in the SWC, defined by

$$
D(q, \omega) \equiv \frac{N}{4 \pi}\left(\frac{d \omega_{m i}^{x y}(q)}{d q_{m}}\right)^{-1}
$$

Using Eq. (1.28) for $\omega_{m}^{x y}(q)$ we obtain

$D(q, \omega)=\frac{N}{2 \pi} \frac{\Theta\left(\omega-\epsilon_{1}^{x y}(q)\right) \Theta\left(\epsilon_{2}^{x y}(q)-\omega\right)}{\left\{\left[\epsilon_{2}^{x y}(q)\right]^{2}-\omega^{2}\right\}^{1 / 2}}$,

a density of states diverging at the upper boundary of the SWC. With Eqs. (1.32) and (1.34) the longitudinal correlation function (1.31) for the $X Y$ model takes the form

$$
S_{z z}(q, \omega)=2 \frac{\Theta(\omega-J \sin q) \Theta(2 J \sin (q / 2)-\omega)}{\left[4 J^{2} \sin ^{2}(q / 2)-\omega^{2}\right]^{1 / 2}},
$$

which is the exact result as is verified by evaluating the formulas of Neimeijer. ${ }^{13}$

We attempt to treat the $\mathrm{HB}$ AF at $T=0$ in a similar way. The sets $\left\{\lambda_{j}\right\}$ of the HB AF SWC are the same as those for the $X Y$ model. Therefore, we again expect to find three spectral branches ( $m=1,3,5)$ for $N=10$. We refer back to Fig. 1 which shows the HB AF SWC states (full triangles) and compare with Fig. 3 for the $X Y$ model. There are two important differences:

(i) Although it is clear that each SWC state belongs to one of the three branches, the finite-chain excitations follow the $N \rightarrow \infty$ branches only approximately, i.e., there is an appreciable finite-size effect for the HB AF.

(ii) Some finite, albeit small, spectral weight appears well above the SWC. No such feature occurs for the $X Y$ model.

In analogy to the $X Y$ case we derive an exact expression for the density of states in the HB AF SWC Eq. (1.27):

$$
D(q, \omega)=\frac{N}{2 \pi} \frac{\Theta(\omega-(\pi / 2) J \sin q) \Theta(\pi J \sin (q / 2)-\omega)}{\left[\pi^{2} J^{2} \sin ^{2}(q / 2)-\omega^{2}\right]^{1 / 2}}
$$


If we now make use of our conjectured analytic expression (1.16) for $S_{z z}(q, \omega)$, we obtain the following analytic expression for the (squared) matrix elements

$M(q, \omega)=\frac{2 A \pi}{N}\left(\frac{\pi^{2} J^{2} \sin ^{2}(q / 2)-\omega^{2}}{\omega^{2}-\left(\pi^{2} / 4\right) J^{2} \sin ^{2} q}\right)^{1 / 2}$.

The consistency and accuracy of our procedure is featured in Fig. 4, which corresponds to Fig. 1, except that the states of the triplet sea have been omitted. The open triangles show the locations of the SWC states for $N=10$. The solid circles represent the locations of the same finite- $N$ states derived from the Bethe ansatz calculations by solving Eq. (1.37) for $\omega$ and putting into the result the $M_{m n}^{*}$ for $N=10$. It turns out that the best value of the constant $A$ for this purpose is $A=1$.

Evidently, the agreement is very good indeed, giving further demonstration of the basic soundness of the approach. We believe this result for matrix elements of the dominant low-lying excitations to have considerable significance, since this is the first theoretical calculation of such an important property of the HB AF.

In Fig. 5 we compare density-of-states functions

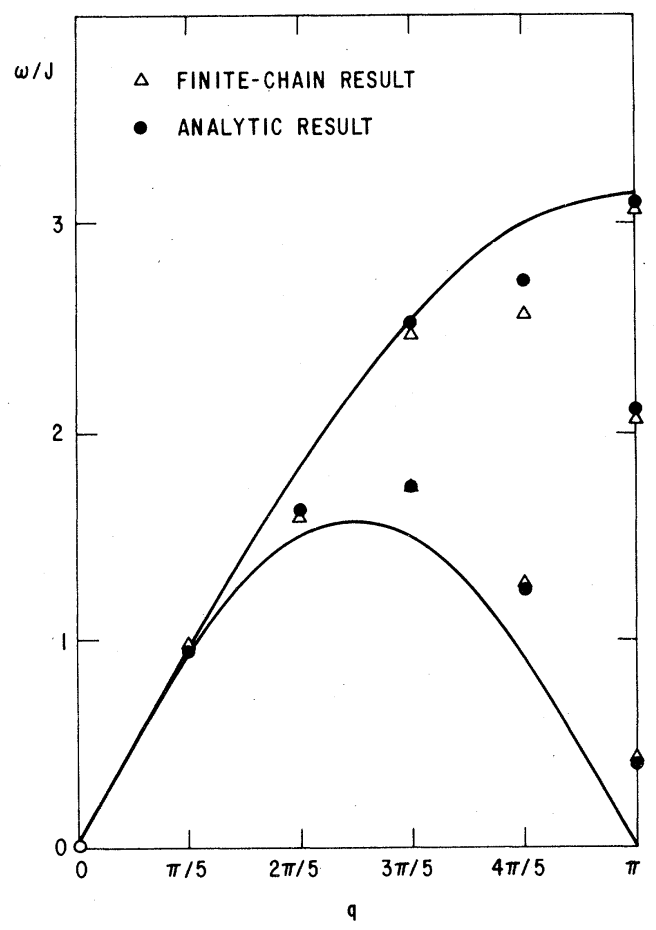

FIG. 4. SWC contributions to $S_{z z}(q, \omega)$ for the $\mathrm{HB} \mathrm{AF}$ as in Fig. 1. The open triangles denote the coordinates in $(q, \omega)$ space of the SWC excitations for $N=10$. The solid circles represent the locations of the same finite- $N$ states derived from our analytic expression for the matrix elements (1.37) as described in the text.

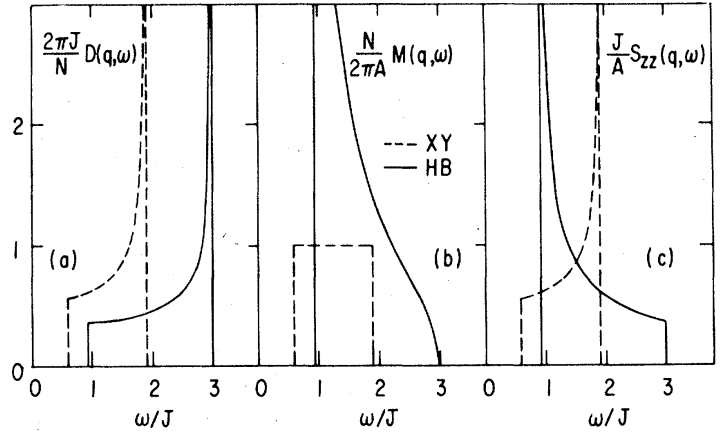

FIG. 5. Frequency dependence at fixed $q=4 \pi / 5$ of three important quantities: (a) normalized density of states in the SWC $(2 \pi J / N) D(q, \omega)$, (b) normalized matrix element expressions $(N / 2 \pi A) M(q, \omega)$, (c) normalized dynamic correlation function $(J / A) S_{z z}(q, \omega)$. The solid curves represent the HB AF and the dashed curves the $X Y$ model, for which the constant $A$ is determined exactly to be $A=2$.

$D(q, \omega)$, matrix-element expressions $M(q, \omega)$ and correlation functions $S_{i z}(q, \omega)$ in the thermodynamic limit for both the $X Y$ and HB AF models, at zero temperature. All functions are evaluated at $q=4 \pi / 5$, which is close to $\pi$. In Fig. 5(a), the solid curve shows the exact (normalized) density of states function $(2 \pi J / N) D(q, \omega)$ of Eq. (1.36) for the HB AF SWC, which is a step function at the lower boundary $\epsilon_{1}(q)$ and diverges at the upper boundary $\epsilon_{2}(q)$. For comparison, we show (dashed) the corresponding exact quantity for the $X Y$ model (1.34), which has the same behavior, but now with boundaries $\epsilon_{1}^{x v}(q)$ and $\epsilon_{2}^{x y}(q)$. In Fig. 5(b), are shown the normalized matrix-element function $(N / 2 \pi A) M(q, \omega)$ for the $X Y$ model (dashed) and the HB AF (solid curve). For the $X Y$ model, for which $A=2$, the matrix elements are constant for all states within the SWC, and hence the $X Y$ dynamical correlation function $(J / A)$ $S_{z z}(q, \omega)$ [shown dashed in Fig. 5(c)] reflects the $X Y$ density of states function, and diverges at the upper boundary of the SWC. For the HB AF, on the other hand, the matrix-element expression $(N / 2 \pi A)$ $M(q, \omega)$ [solid line in Fig. 5(b)] is no longer constant over the SWC, but diverges at the lower boundary $\epsilon_{1}(q)$, and vanishes sufficiently rapidly to cancel the density-of-states divergence, at the upper boundary $\epsilon_{2}(q)$. Consequently, the dynamic correlation function $(J / A) S_{z z}(q, \omega)$ [solid curve in Fig. 5(c)] behaves quite differently from its $X Y$ counterpart and has a divergence at the lower boundary and a finite step at the upper boundary, as discussed in Sec. IC.

It is very interesting, therefore, to conjecture the behavior of $S_{z z}(q, \omega)$ for an anisotropic Heisenberg $X Y$ model. Similar arguments to those just presented suggest strongly $S_{z z}(q, \omega)$ should have a divergence at both the lower and upper boundaries of the appropriate anisotropic SWC, and therefore a double peak 
should be visible in appropriate neutron scattering experiments for a 1D system of this type. A detailed account will shortly be submitted for publication. ${ }^{28}$

\section{E. Estimation of the constant $A$ : sum rules}

A variety of sum rules exist which govern the spin dynamics of both the $X Y$ and HB AF models. For the HB AF these sum rule calculations can be used to demonstrate the rather good accuracy of our approximate result (1.16) and to evaluate the constant $A$. In order to illustrate their consistency and their relations to important static quantities we will apply these sum rules first to the $X Y$ model where exact results are known. Recalling the expression for the $X Y$ longitudinal dynamic correlation function $S_{z z}(q, \omega)$ or Eq. (1.35) we have the following sum rules for $T=0$ :

(i) The static susceptibility is related to $S_{\mu \mu}$ by

$$
\chi_{\mu \mu}(q) \equiv 2 \int_{0}^{\infty} \frac{d \omega}{2 \pi} \omega^{-1} S_{\mu \mu}(q, \omega)
$$

for the $X Y$ model we obtain

$$
\chi_{z z}(q)=\frac{1}{2 \pi J \sin (q / 2)} \ln \frac{1+\sin (q / 2)}{1-\sin (q / 2)}
$$

For small $q$

$$
(q \geqslant 0) \text {. }
$$

$$
\chi_{z z}(q)=\frac{1}{\pi J}+\frac{1}{12 \pi J} q^{2}+\cdots .
$$

This result agrees with the uniform $(q=0)$ susceptibility derived thermodynamically by differentiation of the magnetization with respect to the field ${ }^{13}$ :

$$
\chi_{z z}(0) \equiv \frac{d}{d h}\left(\frac{1}{\pi} \arcsin (h / J)\right)_{h=0}=\frac{1}{\pi J} .
$$

(ii) A second sum rule governs the static susceptibility (integrated intensity):

$$
I_{\mu \mu}(q) \equiv \int_{0}^{\infty} \frac{d \omega}{2 \pi} S_{\mu \mu}(q, \omega) .
$$

For the $X Y$ model this yields

$$
I_{z z}(q)=\frac{|q|}{2 \pi} \text {. }
$$

The function $I_{\mu \mu}(q)$ itself satisfies the sum rule

$$
\int_{-\pi}^{+\pi} \frac{d q}{2 \pi} I_{\mu \mu}(q) \equiv\left\langle\left(s_{l}^{\mu}\right)^{2}\right\rangle=\frac{1}{4},
$$

which is, of course, obeyed by Eq. (1.43).

(iii) A further sum rule involves the first frequency moment:

$$
K_{\mu \mu}(q) \equiv \int_{0}^{\infty} \frac{d \omega}{2 \pi} \omega S_{\mu \mu}(q, \omega)
$$

It is related to a double commutator through

$$
K_{\mu \mu}(q)=-\frac{1}{2}\left\langle\left[\left[H, s^{\mu}(q)\right], s^{\mu}(-q)\right]\right\rangle,
$$

where $H$ is the Hamiltonian of the system under consideration. For the $X Y$ model at $T=0$ this commutator (with $\mu=z$ ) can be evaluated, yielding

$K_{z z}(q)=-\left(E_{G} / N\right)(1-\cos q)=\frac{J}{\pi}(1-\cos q)$,

where $E_{G}=-N J / \pi$ is the ground-state energy. ${ }^{29}$ The same result is, of course, obtained by evaluating the integral (1.45).

The sum rules (i) to (iii) were derived in a paper by Hohenberg and Brinkman. ${ }^{12,30}$ However, we should mention at this stage that this work contains two errors which can mislead those wishing to use their sum-rule prescriptions. The first error is that the factor $\left(\frac{1}{2}\right)$ in front of the double commutator (1.46) is missing. Second, the "static susceptibility" defined by the sum rule corresponding to Eq. (1.38) is actually only half the thermodynamic susceptibility in the limit $q \hookrightarrow 0$. Luckily, these errors do not influence the results because only ratios of the corresponding quantities are discussed, where the two missing factors cancel. However, these errors have influenced our estimate of the constant $A$ in Eq. (1.16) in a preceding publication. ${ }^{20}$ In the following we will, therefore, reproduce these calculationscorrected and in an extended fashion. ${ }^{31}$

Applying the sum rules (1.38), (1.42), and (1.45) to our analytic expression (1.16) for the dynamic correlation function of the $\mathrm{HB} \mathrm{AF}$

$$
\begin{aligned}
S_{z z}(q, \omega)= & \frac{A}{\left(\omega^{2}-\frac{1}{4} \pi^{2} J^{2} \sin ^{2} q\right)^{1 / 2}} \Theta\left(\omega-\frac{\pi}{2} J \sin q\right) \\
& \times \Theta\left[\pi J \sin \left(\frac{q}{2}\right)-\omega\right]
\end{aligned}
$$

yields the SWC results for the corresponding static quantities.

(i) The static susceptibility becomes

$$
\chi_{z z}(q)=\frac{A}{\pi^{2} J} \frac{q}{\sin q} .
$$

The staggered susceptibility $\chi_{z z}(\pi)$ is infinite at $T=0$, as is also the case for the $X Y$ model (1.39).

However, the divergence of the longitudinal susceptibility is stronger for the $\operatorname{HB} \operatorname{AF}\left[X_{z z}(q) \sim(\pi-q)^{-1}\right]$ than for the $X Y$ case $\left[X_{z z}(q) \sim|\ln (\pi-q)|\right]$.

(ii) The static correlation function is given by

$$
I_{z z}(q)=\frac{A}{2 \pi} \ln \frac{1+\sin (q / 2)}{\cos (q / 2)} \quad(q \geqslant 0) .
$$

For small $q$

$$
I_{z:}(q) \simeq \frac{A}{4 \pi} q \quad(q \geqslant 0) .
$$


Note that for $q=\pi, I_{z z}(q)$ has a logarithmic divergence whereas in the $X Y$ model (1.43) it is linear in $q$ and therefore finite at the zone boundary.

(iii) Evaluating the first frequency moment yields

$$
K_{z z}(q)=\frac{1}{4} A J(1-\cos q)
$$

The results $(1.49),(1.50)$, and (1.52) all contain the constant $A$, which has yet to be determined explicitly. Knowing the exact result for the direct (thermodynamic) susceptibility, ${ }^{24.32}$

$$
\chi_{z z}(0)=\frac{1}{\pi^{2} J},
$$

we take the appropriate limit of Eq. (1.49) to obtain

$$
A=1 \text {. }
$$

On the other hand $K_{z z}(q)$ may independently be evaluated from the commutator (1.46), yielding for the HB AF

$$
K_{z z}(q)=-\frac{2}{3}\left(E_{G} / N\right)(1-\cos q),
$$

where $E_{G}=-N\left(\ln 2-\frac{1}{4}\right)$ is the ground-state energy. ${ }^{25}$ Comparison with Eq. (1.52) shows that the $q$ dependence is exactly reproduced by our SWC result and gives

$$
A=\frac{4}{3}\left(2 \ln 2-\frac{1}{2}\right) \simeq 1.1817 \ldots .
$$

For the integrated intensity $I_{z z}(q)$ there exist no exact results which could directly be compared with Eq.
(1.50) in order to determine $A$. However, finitechain extrapolations for small $q(=2 \pi / N)$ are consistent with Eq. (1.51) indicating the value

$$
A=1.1 \text {. }
$$

Finally application of sum rule (1.44) on Eq. (1.50) yields

$$
A=\frac{\pi^{2}}{8 G}=1.3469 \ldots,
$$

where $G=0.9159 \ldots$ is Catalan's constant.

Additional information on $A$ may be obtained from a study of the static correlation function in real space

$$
\tilde{I}_{z z}(R)=\int_{-\pi}^{+\pi} \frac{d q}{2 \pi} e^{-i q R} I_{z z}(q) \text {. }
$$

This integral applied to Eq. (1.50) may conveniently be divided into two parts. Evaluation of the first part gives (denoting $q^{*} \equiv \pi-q$ )

$$
\begin{aligned}
\tilde{I}_{::}^{(1)}(R) & =\frac{A}{2 \pi^{2}}(-1)^{R+1} \int_{0}^{\pi} d q^{*} \cos q^{*} R \ln \sin \frac{q^{*}}{2} \\
& =\frac{A}{4 \pi} \frac{(-1)^{R}}{R}
\end{aligned}
$$

The second part can be expressed in terms of an infinite sum of powers of $R^{-2}$, plus a contribution which is nonzero only for the autocorrelation function $^{33}$ :

$$
\begin{aligned}
\tilde{I}_{z z}^{(2)}(R) & =\frac{A}{2 \pi^{2}}(-1)^{R} \int_{0}^{\pi} d q^{*} \cos q^{*} R \ln \left(1+\cos \frac{q^{*}}{2}\right) \\
& =\frac{A}{2 \pi} \delta_{R, 0}+A \sum_{n=1}^{\infty} R^{-2 n} \sum_{k=n}^{\infty}(-1)^{k+n-1} \frac{(2 n-1) !\left(\begin{array}{c}
2 k \\
2 n-1
\end{array}\right) \Pi^{2 k-1-2 n}\left(2^{2 k}-1\right) B_{2 k}}{k(2 k) ! 2^{2 k+1}} .
\end{aligned}
$$

The $B_{2 k}$ are Bernoulli numbers. By studying this expression for special cases of $R$ we can derive more values for the constant $A$.

(i) $R=0$ : The expression for $A$ obtained here is equivalent to the sum rule (1.44) on $I_{z z}(q)$, and hence gives us the value (1.58)

$$
A=1.3469 \ldots \text {. . }
$$

(ii) $R=1$ : For this case it is possible to evaluate the integral (1.59) directly, avoiding the infinite sum. ${ }^{33}$ The result is

$$
\tilde{I}_{z z}(1)=\frac{A}{\pi^{2}}
$$

But $\tilde{I}_{z z}(1)$, the nearest-neighbor correlation function, is proportional to the ground-state energy. Hence we obtain a new value

$$
A=1.4579 \ldots \text {. . }
$$

(iii) $R \rightarrow \infty$ : The asymptotic behavior of Eq.

(1.61) for large $R$ is

$$
\tilde{I}_{z z}(R)=\frac{A}{4 \pi} \frac{(-1)^{R}}{R}+O\left(R^{-2}\right)
$$

This reflects the strong short-range order of the antiferromagnetic ground state. An extrapolation using results of Bonner and Fisher ${ }^{34}$ indicates that

$$
A \geq 1.45 \text {. }
$$

We have compiled the various results for $A$ in Table I. 
TABLE I. Determination of the constant $A$ in Eq. (1.48) by various sum rules. The first column denotes the corresponding static quantities which are involved. The second column indicates their relation to the dynamic quantity $S_{z z}(q, \omega)$, and the last column gives the resulting value for $A$.

\begin{tabular}{llc}
\hline \hline $\begin{array}{c}\text { Static } \\
\text { quantity }\end{array}$ & $\begin{array}{c}\text { Relation to } \\
S_{z z}(q, \omega)\end{array}$ & $\begin{array}{c}\text { Value of } \\
\text { constant } A\end{array}$ \\
\hline$\chi_{z z}(0)$ & $\int d \omega \omega^{-1} q \rightarrow 0$ & 1. \\
$I_{z z}(0)$ & $\int d \omega q \rightarrow 0$ & $\simeq 1.1$ \\
$E_{G} / N$ & $\int d \omega \omega$ any $q$ & 1.1817 \\
$\left\langle\left(s_{i}\right)^{2}\right\rangle$ & $\int d \omega \int d q$ & 1.3469 \\
$\left\langle s_{i} s_{i+R}\right\rangle$ & $\int d \omega \int d q e^{i q R}$ & $z 1.45$ \\
\hline \hline
\end{tabular}

Clearly, if our expression (1.48) for $S_{z z}(q, \omega)$ were exact, all sum rules should yield the same value of $A .^{35}$ In principle, two effects can give rise to the discrepancy: (i) The description of the contribution of the SWC to $S_{z z}(q, \omega)$ by Eq. (1.48) is only approximate. (ii) There exists a continuum of higher triplet excitations ("triplet sea") which also contributes to $S_{z z}(q, \omega)$. We conjecture that this latter contribution constitutes the main deviation from our approximate description. Defining a kind of "average frequency"

$$
\tilde{\omega}_{z z}^{2}(q) \equiv \frac{K_{z z}(q)}{\chi_{z z}(q)},
$$

Hohenberg and Brinkman, ${ }^{12}$ by using exact values for $K_{z z}(q)$ and $\chi_{z z}(0)$, show that

$$
\tilde{\omega}_{z z}^{2}(q)=1.1817 \epsilon_{1}^{2}(q)[1+O(q)],
$$

implying that most of the spectral weight for small $q$ is concentrated near the lower threshold $\epsilon_{1}(q)$ $=(\pi / 2) J \sin q$. This result agrees with our numerical extrapolations of the same quantity for finite chains. ${ }^{27}$

Since the neglected triplet-sea states have relatively large energy, their effect (for a given $q$ ) is minimal for the $\omega^{-1}$ moment leading to the minimum value $A=1$. Their influence becomes more important for the $\omega^{0}$ moment and maximal for the $\omega^{-1}$ moment (always in the limit of small $q$ ). Apparently the relative weight of the triplet-sea states increases with $q$, becoming maximal at the zone boundary $q=\pi$. This is clearly reflected in the last two rows of Table $I$. The sum rule for $\left\langle\left(s_{i}^{i}\right)^{2}\right\rangle$ averages the triplet-sea effect over the whole Brillouin zone and, therefore, provides a larger constant $A=1.3469$ than the same frequency moment for small $q$ only $(A \simeq 1.1)$. Furthermore, since the real-space correlation func- tion $\tilde{I}_{z z}(R)$ is dominated by the $q=\pi$ modes (especially for large $R$ ), it is expected to yield the largest value of the constant $A$, as is the case. Hence, we seem to have a consistent picture for the relative importance of the dominant SWC contribution and the small but finite contribution of the triplet sea. We have also been able to determine the constant $A$ independently of the various sum rules: By extrapolation of finite- $N$ matrix elements for $q=\pi$ and $\pi-(2 \pi / N)$, using the expression (1.37),

$$
A \geq 1.35 \text {. }
$$

In conclusion we should point out that, although $A$ cannot be determined uniquely, our expression (1.48) is the first result for $S_{z z}(q, \omega)$ of the HB AF which can be related to sum rules and gives reasonable results for all $q$.

We should at this point give some discussion of these triplets whose presence is required to satisfy the sum rules, but whose effect on the spin dynamics does not seem to be very perceptible. They cannot be characterized by our extension of des Cloizeaux and Pearson's prescription for the Bethe ansatz states. ${ }^{4,8}$ The same is the case with the singlet continuum which becomes degenerate with the triplet SWC in the thermodynamic limit. An extended Yang-Sutherland prescription ${ }^{36}$ can accommodate both singlet and triplet SWC's, but gives no account of the triplet sea. At present, therefore, we have no theoretical tool available for examining them. There is currently some discussion on the role of bound spin complexes and soliton excitations in the $\mathrm{HB}$ AF. ${ }^{37}$ It is worth noting that it follows directly from the prescription for the class $C$ of Bethe ansatz states $^{24}$ that the SWC states are clearly of a spin-wave character. Hence, we may comment that at very low temperatures, where the SWC contributions are expected to dominate the spin dynamics, neither bound states nor solitons are likely to be observable in the 1D quantum Heisenberg antiferromagnet (1.4) using current experimental (neutron scattering) techniques. It might, however, be interesting to determine whether the states of the triplet sea have any special (non-spin-wave) character. Subsequent investigations in Sec. II show the presence of non-SWC states with appreciable spectral weight near the critical field. We have been able to identify such states as bound states of two overturned spins which are familiar from the case of the zero-field HB ferromagnet.

As a further comment we remark that Griffiths studied thermodynamic properties of the special class $C$ of Bethe ansatz states and the results are available as unpublished reports. ${ }^{38}$ However, as he pointed out, they are too small in number to influence the partition function in the thermodynamic limit, at least for zero field. Now we realize that they dominate the low-temperature spin dynamics in zero field, and also in nonzero field as we shall see in Sec. II. It is in- 
teresting to complete our sum-rule discussion with a brief account of the classical spin-wave results. The fluctuations of the Néel ground state are described by sharp spin waves perpendicular to the order parameter as follows ${ }^{1.2}$ :

$$
S_{\perp}(q, \omega)=A^{\prime} \tan \frac{q}{2} \delta(\omega-J|\sin q|),
$$

where $A^{\prime}$ is another normalizing constant. Application of sum rule (1.45) to Eq. (1.71) yields

$$
K_{\perp}(q)=\frac{A^{\prime} J}{2 \pi}(1-\cos q),
$$

which has the same $q$ dependence as the quantum result (1.55). This is due to the fact that the $q$ dependence of the double commutator in Eq. (1.46) is independent of spin quantum number and is therefore also valid in the (classical) limit $s=\infty$. The perpendicular static correlation function of the Néel ground state is obtained by sum rule (1.42):

$$
I_{\perp}(q)=\frac{A^{\prime}}{2 \pi} \tan \frac{q}{2} .
$$

It diverges as $(\pi-q)^{-1}$ whereas our result (1.50) for the $s=\frac{1}{2}$ HB AF diverges more weakly as $|\ln (\pi-q)|$. Both curves are shown in Fig. 6 togeth-

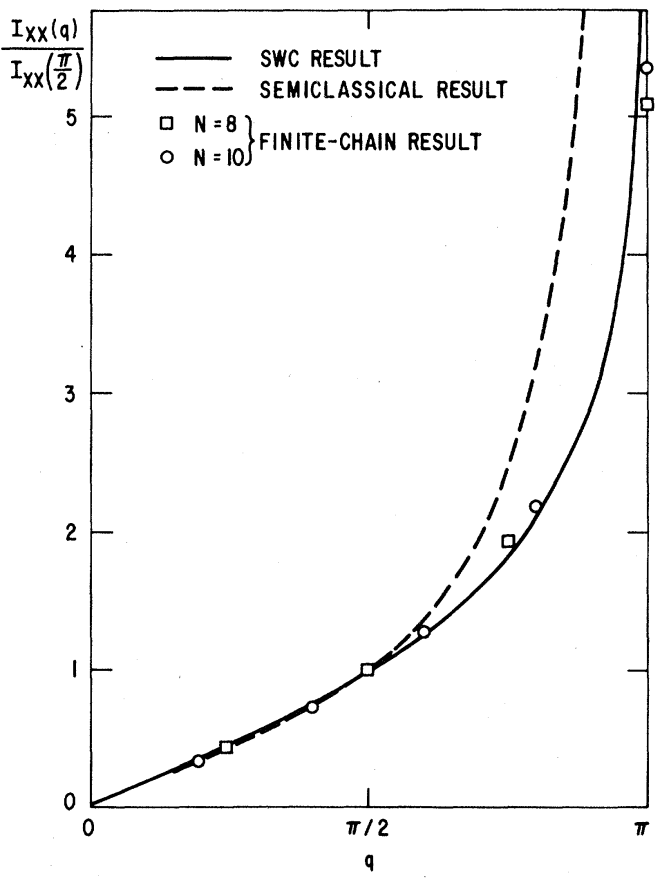

FIG. 6. Normalized $q$-dependent static correlation function for the $\mathrm{HB}$ AF at $T=0$. The solid line represents the SWC result (1.50). It is in good agreement with exact calculations on cyclic chains containing $N=8$ (squares) and $N=10$ (circles) spins. For comparison the result of the two-sublattice theory (1.73a) is shown (dashed line). It deviates markedly from (1.50) for $q>\pi / 2$. er with (exact) finite-chain results for $N=8$ and 10 . The latter clearly follow the SWC curve. The perpendicular static susceptibility

$$
\chi_{\perp}(q)=\frac{A^{\prime}}{2 \pi J} \frac{1}{1+\cos q}
$$

as obtained by sum rule (1.38), is identical to the result for the entirely classical chain obtained by means of the transfer operator technique..$^{39.40}$ It has a strong divergence of the type $(\pi-q)^{-2}$ which has to be contrasted with the weaker divergence $(\pi-q)^{-1}$ of our SWC result (1.49) reflecting the isotropy in spin space.

\section{F. Comparison with other theories}

Our approach, which leads to the (approximate) analytic expression (1.48) for the dynamic correlation function $S_{\mu \mu}(q, \omega)$ at $T=0$, is completely new, comprising the following advantages:

(1) It deals explicitly with spin excitations instead of the rather abstract "particle" and "particle-hole" excitations in the fermion representation. ${ }^{17.18}$ Therefore the underlying physics is much more transparent than in the fermion picture and is directly applicable to the selection rules involving spin operators.

(2) In contrast to most (approximate) approaches, 1.2 .17 .18 it conserves the rotational symmetry in spin space, which is reflected in the equivalence of longitudinal $(\mathrm{II} z)$ and transverse $(\perp z)$ fluctuations.

(3) In contrast to semiclassical calculations $s^{1.2 .19}$ it treats the extreme $s=\frac{1}{2}$ quantum limit at the outset and therefore reveals the quantum behavior in a fundamental way and not merely as a correction to classical behavior. This aspect is particularly important for nonzero magnetic field (see Sec. II).

(4) A unique aspect of our approach is that it clearly distinguishes between effects of density of states and effects of matrix elements. This is very useful for elucidating problems arising in theories which are based on linearized dispersion relations in the fermion picture and therefore suppress important density-of-states effects. ${ }^{41.42}$

(5) It employs an analytic expression for $S_{\mu \mu}(q, \omega)$ which is applicable (for all $q$ ) to the set of sum rules presented in Sec. IE. These in turn give results for $q$-dependent static quantities which are in good agreement with the few available exact results despite some uncertainty in the constant $A$ due to the approximate character of Eq. (1.48). All other dynamics calculations we are aware of have provided results which are either not applicable ${ }^{19}$ to the sum rules, applicable only for a limited range of $q$ values ${ }^{41}$ or if applied yield results which are in striking contradiction to exact results. ${ }^{17}$

(6) It reproduces, for any $q$, those characteristic features which have been observed by neutron 
scattering experiments on appropriate quasi-1D compounds at very low $T .^{16,43}$

Nevertheless, we discuss here briefly three other theoretical approaches to the $T=0$ dynamics which preceded our calculations. Each one provides results which show at least some aspects of the characteristic behavior (1.48) as described above.

(i) Todani and Kawasaki ${ }^{17}$ employed a Green'sfunction approach in the fermion representation. They treated the $x x$ and $y y$ part of the exchange interaction exactly and the $z z$ part, which transforms into a fermion interaction, in the Hartree-Fock approximation. Their result for $S_{z z}(q, \omega)$ is governed by the "particle-hole" excitations which form a twoparameter continuum equivalent to our SWC, with boundaries similar to our $\epsilon_{1}(q)$ and $\epsilon_{2}(q)$ of Eq. (1.2). However, instead of a divergence at the lower boundary and a tail out to $\epsilon_{2}(q)$ their result is a smooth curve with a maximum somewhat above the lower boundary and zeros at both boundaries, yielding therefore an "effective dispersion curve" which has a gap at $q=\pi$. The defect of their result is best understood when we discuss it in relation to Fig. 5 . The density-of-states effect $D(q, \omega)$, which is qualitatively the same for both the $X Y$ model and the HB AF is treated essentially exactly in their approach. However, the matrix element effect $M(q, \omega)$, which is strongly dependent on the $z z$-exchange interaction appears to be only poorly approximated by the Hartree-Fock method.

(ii) Mikeska ${ }^{19}$ studied the HB AF dynamics by a Holstein-Primakoff-type approach. In order to avoid the problems for $1 \mathrm{D}$ magnets introduced by the ordinary Holstein-Primakoff transformation, ${ }^{44}$ which assumes a preferred direction in spin space, Mikeska introduces a representation of spin operators in terms of boson operators which uses only rotationally invariant combinations. This accounts for the absence of LRO. He obtains an analytic expression only for $q \leq \pi$ :

$$
S_{\mu \mu}(q, \omega) \propto \frac{1}{\left\{\omega^{2}-\left[\epsilon^{\mathrm{CL}}(q)\right]^{2}\right\}^{\alpha}} \Theta\left(\omega-\epsilon^{\mathrm{CL}}(q)\right) .
$$

$\epsilon^{\mathrm{CL}}(q)$ is the classical spin-wave frequency (1.1) and the exponent is given by $\alpha=1-\pi^{-1}[s(s+1)]^{-1 / 2}$. since the result is predicted to be valid to order $1 / s$ the value of the exponent in the $s=\frac{1}{2}$ quantum limit, $\alpha=0.63$, is in reasonably good agreement with our $\alpha=0.5$. Note that Eq. (1.74) has no upper cutoff violating therefore at least sum rule (1.55). Mikeska and Pesch extended this approach to smaller $q$ by a numerical investigation. ${ }^{45}$ Their result predicts for $q \leqslant \pi / 2$ two peaks in $S_{\mu \mu}(q, \omega)$, one peak at $\epsilon^{\mathrm{CL}}(q)$ and a second one at some higher energy. However, the existence of this second peak appears to us to be in doubt. In the approach to be discussed next, ${ }^{41} \mathrm{a}$ similar phenomenon appears, which is clearly an unphysical byproduct of the linearized dispersion relation.

(iii) As is well known, the 1D $s=\frac{1}{2} \mathrm{HB}$ AF Hamiltonian (1.4) can be mapped onto an interacting fermion Hamiltonian by means of the Jordan-Wigner transformation. The continuum limit (Luttinger model) is obtained by linearizing the dispersion relation of the fermion excitations with respect to $q$. In this limit the system can be treated exactly according to the method of Luther and Peschel. ${ }^{41}$ By Fourier transforming their result for the time-dependent pair-correlation function we obtain ${ }^{46}$

$$
S_{z z}(q, \omega) \propto\left\{\begin{array}{l}
\omega \delta(\omega-c q), \quad q \leq 0 \\
{\left[\omega^{2}-c^{2}(\pi-q)^{2}\right]^{-1 / 2} \Theta(\omega-c(\pi-q)), \quad q \leq \pi}
\end{array}\right.
$$

Here, $c$ is a renormalized Fermi velocity which is assigned the value $c=(\pi / 2) J$ in order to reproduce the correct lower boundary of the SWC near $q=0$ and $\pi$. The correct value $\frac{1}{2}$ of the exponent is obtained by using Baxter model results. ${ }^{41}$ The linearized fermion dispersion of the continuum approximation is clearly echoed in Eq. (1.75) by linearized spin-wave dispersion curves. Therefore, we expect the results of this approach to be valid only for small excitation energies $\omega$, which occur in the present case for $q \geq 0$ and $q \leq \pi$. For small $q$ the narrow SWC of Eq. (1.48) and Fig. 2 is approximated by a $\delta$ function. It nicely reproduces the linear term in time static correlation function (1.50) and the finite limit of the static susceptibility (1.49) for $q \rightarrow 0$. For $q \leqslant \pi$, (1.75) yields the characteristic square-root behavior of Eq. (1.48) but with the lower boundary of the SWC replaced by a straight line and without the important upper cutoff. Although the validity of this approach is clearly restricted to small frequencies, the two parts of Eq. (1.75) are commonly written as a sum of two terms, ${ }^{41,42}$ and each term is given a physical meaning throughout the whole Brillouin zone. It is this interpretation which would lead to a double-peak prediction for $S_{z z}(q, \omega)$ at small $q$ and is referred to by Mikeska and Pesch. ${ }^{45}$ At the same time it leads to results which are in serious violation of the sum rules of Sec. II E. Its inadequacy is clearly demonstrated by comparing the corresponding Luther-Peschel result for the $X Y$ model $^{41}$

$$
S_{z z}(q, \omega) \propto\left\{\begin{array}{l}
\omega \delta(\omega-c q), \quad q \geq 0 \\
\Theta(\omega-c(\pi-q)), \quad q \leq \pi
\end{array}\right.
$$


with the exact result (1.35). Here the Fermi velocity is $c=J$. For $q \geq 0$ the narrow SWC is again well approximated by the $\delta$ function, and for $q \leq \pi$ the continuum result (1.76) reflects the step at the lower boundary of the SWC. This second term in Eq. (1.76) has obviously no physical meaning at small $q$. Again the structure of $S_{z z}(q, \omega)$ at higher frequencies is not reproduced at all in the Luttinger model.

\section{G. Finite-temperature effects and experimental comparisons}

As noted in Sec. I A a tail of spectral weight above the lower threshold energy $\epsilon_{1}(q)$ should give rise to an asymmetry in the line shape of the corresponding neutron scattering peaks, since the inelastic-scattering cross section is proportional to $S_{\mu \mu}(q, \omega)$. Such an asymmetry is clearly evident in our SWC result (1.48) for wave numbers near the zone boundary. It is important to verify, however, that this asymmetry effect extends to finite temperatures. Early neutron scattering experiments by Endoh et al. ${ }^{16}$ were done at a reduced temperature $k_{B} T / J \simeq 0.05$ and more recent experiments of Heilmann et al. ${ }^{43}$ at an even lower one: $k_{B} T / J \simeq 0.045$. Since our analytic calculations are valid only for $T=0$, we must resort to finitechain calculations alone to study finite-temperature dynamics. However, as we have demonstrated repeatedly in this section, finite-chain results are a very good indicator of behavior in the thermodynamic limit. The numerical results for $S_{\mu \mu}(q, \omega)$ at finite $T$ are most suitably presented as histograms at fixed $q$. The corresponding interval width has to be chosen large enough in order that finite-size effects are suppressed but small enough in order that the physical structure is not smeared out. This is possible for $N=10$ if the temperature is not too low: $k_{B} T / J \geq 0.3$. Hence in Fig. 7 we have plotted spectral-weight histograms for a variety of $q$ values, and at the two reduced temperatures $k_{B} T / J=0.35$ and 0.5 . It is evident in Fig. 7 that a rather appreciable amount of spectral weight develops at energies below the lower edge $\epsilon_{1}(q)$ of the SWC. For the higher temperature $\left(k_{B} T / J=0.5\right)$, in fact, this part of $S_{z z}(q, \omega)$ with $\omega<\epsilon_{1}(q)$, which we may call the "diffusive tail," is comparable in spectral weight to the part at $\omega>\epsilon_{1}(q)$, which represents the nonclassical asymmetry effect or "quantum tail." The occurrence of both a diffusive tail and a quantum tail may explain why the quantum asymmetry effect was hard to detect unambiguously in the earlier experiments of Endoh et al. ${ }^{16}$ and has only recently been confirmed by further experiments. ${ }^{43}$ Figure 8 shows the temperature dependence of the integrated intensity for various $q$ as obtained by finite-chain calculations with $N=8$ and 10 . The values for $T=0$ reflect the curve of Fig. 6. The value for the $q=\pi$ contribution,
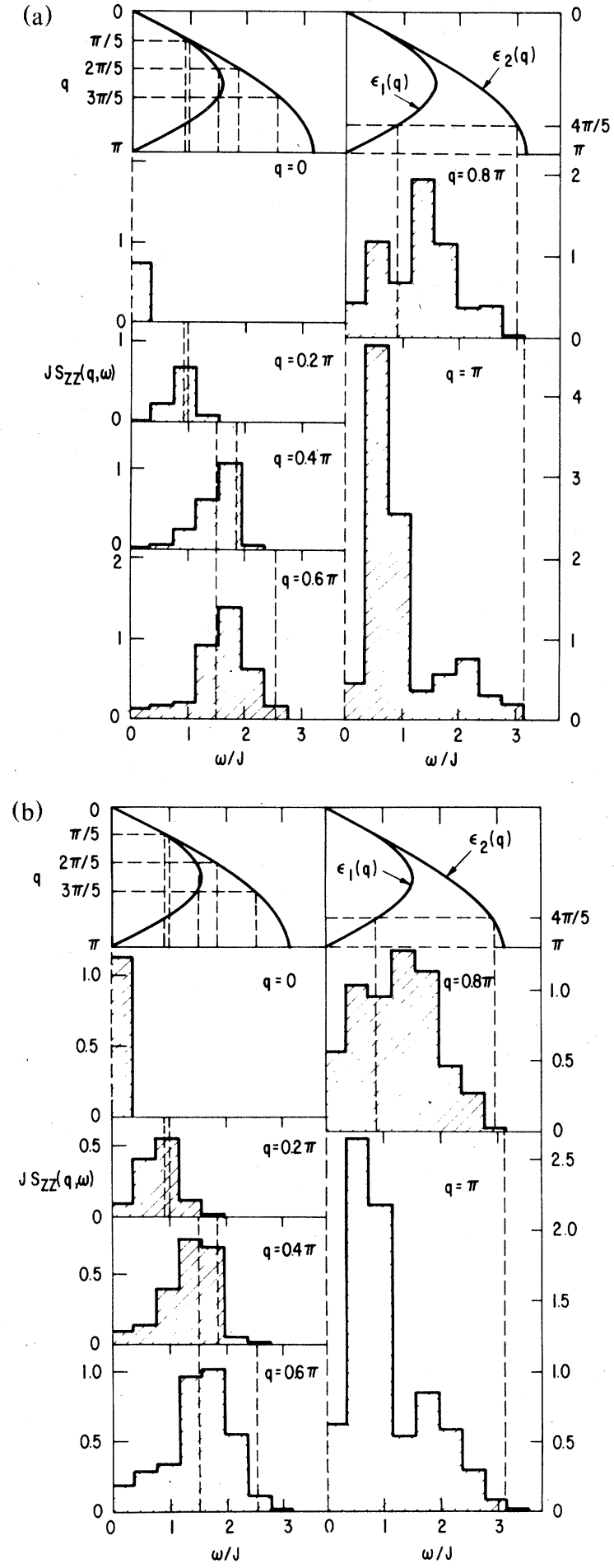

FIG. 7. $S_{z z}(q, \omega)$ for a finite HB AF chain with $N=10$ at finite temperatures, plotted as histograms for fixed $q$ with interval width $\Delta \omega / J=0.4$. The reduced temperatures are in (a) $k_{B} T / J=0.35$ and in (b) $k_{B} T / J=0.5$. A link to our $T=0$ analytic result of Fig. 2 is provided by the SWC boundaries shown at the top. Note the different vertical scales in (a) and (b). 


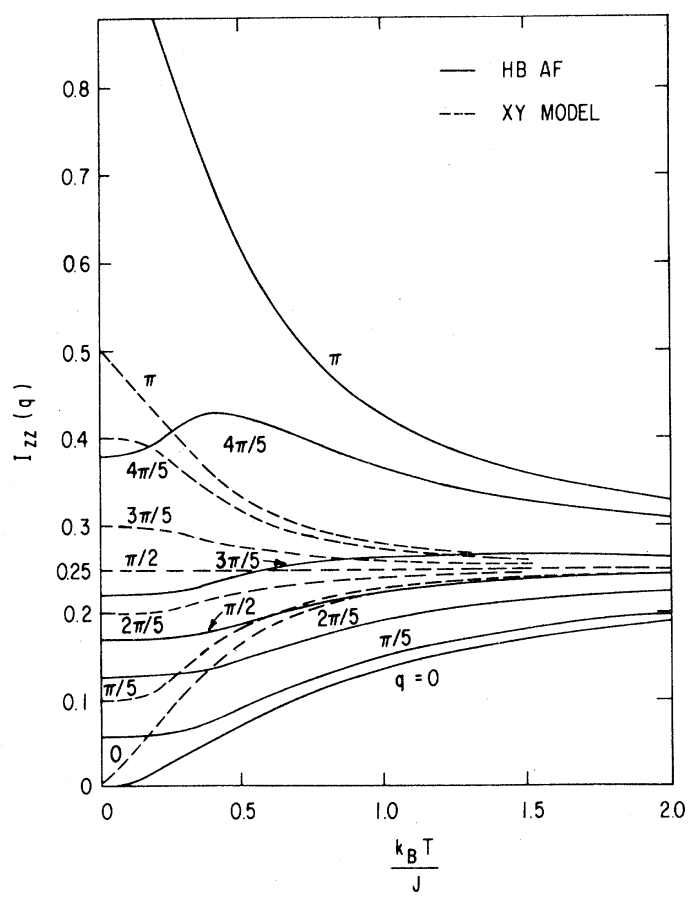

FIG. 8. Temperature dependence of $I_{z z}(q)$ for the HB AF (solid curves) as obtained by finite-chain calculations with $N=8$ and 10 , and the $X Y$ model (dashed curves, exact). The wave numbers corresponding to each curve are shown.

though finite for $N=10$, is predicted to be infinite in the thermodynamic limit. This clearly indicates that the magnetic structure is governed by the staggered mode. With rising $T$ this predominance is increasingly destroyed by thermal fluctuations and the spectral weight is redistributed over the other $q$ 's. The striking feature, however, is that the contributions from $\pi / 2<q \leqq \pi$, which are already large at $T=0$, initially increase with $T$, having a maximum at some finite $T$, then decrease again to the high-temperature limit. This shows that the onset of thermal motion which softens the antiferromagnetic structure has the effect of enhancing the modes with wave numbers just below $q$. The remarkable fact is that the onset of a small magnetic field parallel to the $z$ axis has a similar effect as will be shown in Sec. II (see Fig. 20).

In the high-temperature limit when all pair correlations are suppressed by thermal noise the integrated intensity becomes independent of $q$ :

$$
\lim _{T \rightarrow \infty} I_{z z}(q)=\frac{1}{4}
$$

The corresponding quantities for the $X Y$ model, shown dashed in Fig. 8, were calculated exactly. ${ }^{15}$ The (integrated) spectral weight is finite for all $q$ and is smoothly redistributed with increasing $T$. Note

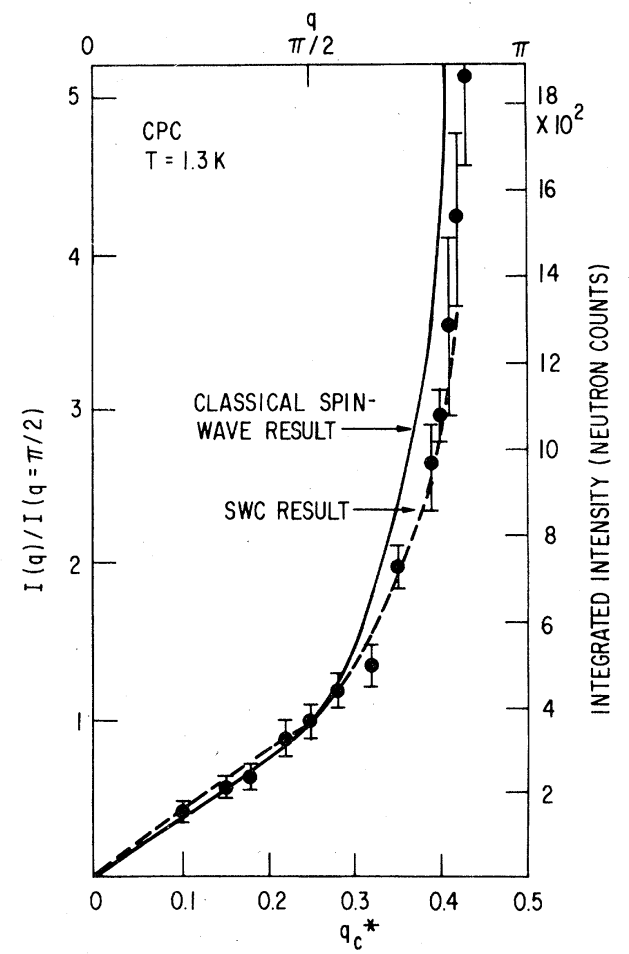

FIG. 9. Integrated intensity $I_{x x}(q)$ normalized to unity at $q=\pi / 2$. Data points for CPC at $T=1.3 \mathrm{~K}\left(k_{B} T / J=0.05\right)$ are from Ref. 16. Predictions for $T=0$ are shown for our SWC approach (dashed curve) and the Anderson theory (solid curve), both multiplied by an experimental correction factor.

that in the Bulaevskii approximation ${ }^{18}$ the result for the HB AF is qualitatively the same as for the $X Y$ model. ${ }^{17}$ The Bulaevskii method, which treats the HB AF as an effective $X Y$ model, is thus insensitive to the effects described above.

In Fig. 9 we compare our SWC result (1.50) for $I_{z z}(q)$ with experimental data of Endoh et al. ${ }^{16}$ for CPC at the low reduced temperature $k_{B} T / J=0.05$. The experimental data points lie consistently below the curve (1.73a) predicted by the Anderson theory but are in quantitative agreement with our result (1.50) out to $q=0.8 \pi$. For still larger $q$ the situation is indeterminate because of the large error bars on the data. It may seem that in this region our $T=0$ result lies somewhat below the data. However, this difference can easily be accounted for by the finite- $T$ enhancement of the modes with $q$ near $\pi$.

\section{DYNAMICS IN A MAGNETIC FIELD}

\section{A. Preliminary survey}

Spin dynamics in nonzero magnetic field has received very little attention so far. This topic is of 
current experimental interest, however, as a result of very recent neutron scattering experiments on the $s=\frac{1}{2}$ linear HB AF CPC ${ }^{43}$ and on the $s=\frac{5}{2}$ linear HB AF TMMC, ${ }^{47}$ both in a field of $70 \mathrm{kOe}$. Active experimental investigation is also underway of proton spin-lattice relaxation times in the $s=\frac{1}{2}$ linear $\mathrm{HB}$ AF compounds $\mathrm{CuSeO}_{4} \cdot 5 \mathrm{H}_{2} \mathrm{O}, \mathrm{CuSO}_{4} \cdot 5 \mathrm{H}_{2} \mathrm{O},{ }^{48-50}$ and $\alpha$-CuNSal, ${ }^{51-53}$ at low temperatures and over a magnetic field range up to and larger than the antiferromagnetic critical field. The theoretical interpretation of these studies requires knowledge of dynamical spin-correlation functions. Finally, a new family of magnetoelastic $s=\frac{1}{2}$ linear HB AF systems has been discovered, ${ }^{54-56}$ which display a spin-Peierls transition from high-temperature uniform-chain behavior to a low-temperature phase where the Heisenberg chains are alternating or dimerized in character as a result of an underlying lattice distortion. Such systems show unusual phase behavior in an applied magnetic field, and spin-dynamical experiments would provide valuable information on the nature of this behavior.

At present, very little theoretical information is available on the low-temperature quantum spin dynamics of the $s=\frac{1}{2} \mathrm{HB}$ AF in an applied magnetic field. The predictions of the classical spin-wave theory ${ }^{57}$ are shown in Fig. 10. At $h=0$ the ground state is of the Néel type with spins, say, parallel to the $z$ axis owing to an infinitesimal anisotropy field.
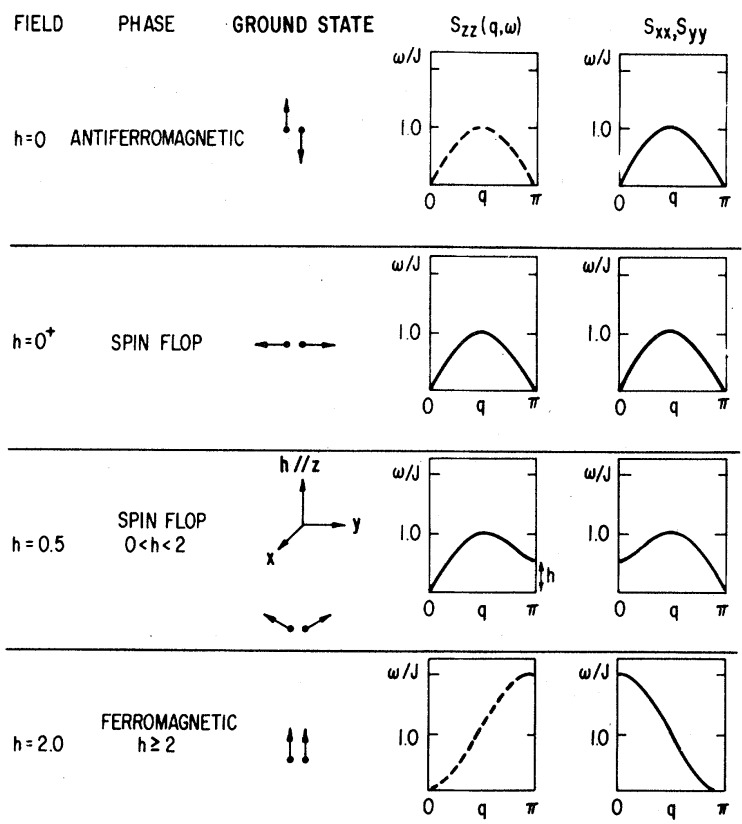

FIG. 10. Spin waves of the classical antiferromagnetic chain. The arrows denote the ground-state configuration of spins belonging to different sublattices. Note that the dispersion curves are shown in the extended Brillouin zone of the classical system.
The spin-wave excitations with the sinelike dispersion Eq. (1.1) are then perpendicular to $z$; i.e., they contribute only to $S_{x x}(q, \omega)$ and $S_{y y}(q, \omega)$. As soon as a very small field is turned on, the classical AF undergoes a transition to the spin-flop phase with spins essentially perpendicular to the field. The rotational symmetry with respect to the $z$ axis is broken and the spins lie, for example, in the $x z$ plane. Now, the spin waves predominantly contribute to $S_{z z}(q, \omega)$ and $S_{y v}(q, \omega)$. With increasing $h$ the dispersion spectrum progressively distorts and develops a gap at $q=0$ for the spin waves with magnetization perpendicular to the field and at $q=\pi$ for the spin waves with magnetization parallel to the field, respectively. The two dispersion curves differ by a reflection about the axis $q=\pi / 2$. When the field reaches the critical field, $h=h_{c}=2$, the system changes to the ferromagnetic phase with all spins aligned parallel to the field. Here, the ferromagnetic spin waves with the familiar cosinelike dispersion contribute exclusively to $S_{x x}(q, \omega)$ and $S_{y y}(q, \omega)$.

In the extreme quantum limit $\left(s=\frac{1}{2}\right)$ the situation is quite different. At $h=0$ the ground state is a singlet. It is completely invariant under rotations in spin space. Hence, the longitudinal and the transverse fluctuations are equivalent (as discussed extensively in Sec. I). When a small field is turned on, although the quantum system does not change to a spin-flop phase, the reduction of rotational symmetry gives rise, nevertheless, to anomalies in the behavior at $h>0$, as we shall see later in this section.

A transverse spin-wave dispersion curve for the quantum chain in nonzero field was first obtained by Pytte $^{58}$ who studied the $s=\frac{1}{2}$ HB AF in the fermion representation and used the Hartree-Fock (Bulaevskii) approximation ${ }^{18}$ for the fermion interaction. He identified the transverse spin waves with singleparticle (or -hole) excitations. His results are in qualitative agreement with the results of a more recent calculation by Ishimura and Shiba ${ }^{59}$ who used a Bethe ansatz technique. In analogy with the work of des Cloizeaux and Pearson ${ }^{4}$ they identified the transverse spin waves with a special subclass of states in the Bethe formalism. The energies of the states were found by solving the Hulthén integral equation numerically (see Appendix B). The important feature that both approaches have in common is the existence of a zero-frequency mode which progressively moves from the zone center $(q=0)$ to the zone boundary $(q=\pi)$ as $h$ increases from zero to $h_{c}$. This feature, which appears also in our own calculations (for the transverse excitations), means that the extreme quantum limit of the HB AF is very different from the classical limit. This concerns not only the details of dynamical correlation functions, as demonstrated in Sec. I, but even such a general 
feature as the lowest dispersion branch for nonzero $h$. The physical relevance of this "spin-wave" branch, i.e., its observability in dynamical experiments is, however, not clarified by either of the two approaches. For this purpose it is necessary to know the appropriate matrix elements between the ground state and these spin-wave excitations. To the best of our knowledge there are no such calculations for the HB AF at nonzero $h$ previous to this study, except for some calculations of frequency moments of dynamic correlation functions for $q \simeq 0$ by Puga. ${ }^{60}$ Our aim in Sec. II is to provide a comprehensive discussion of the longitudinal and the transverse spin dynamics of the $S=\frac{1}{2}$ linear HB AF in a uniform magnetic field and at low temperatures, particularly at $T=0$.

In Sec. II B we present the exact result for $S_{z z}(q, \omega)$ for the $X Y$ model at $T=0$ and arbitrary $h$, which is highly suggestive for our study of the HB AF system. In Sec. II C we derive the energies of various continua of states, which are expected to play an important role in the $T=0$ dynamics. Calculational details are placed in Appendixes A and B. New selection rules, which are valid for macroscopic systems at $h \neq 0$ are introduced and proved in Sec. II D. In Secs. II E and II F we discuss in detail the field dependence of the $T=0$ longitudinal and transverse structure functions. Section II G, finally, deals with experimental comparison and predicts characteristic features which should be observable in future experiments.

\section{B. Exact result for $S_{z z}(q, \omega)$ of the $X Y$ model at $T=0$ and $h \neq 0$}

In Sec. I D we have obtained the exact result for $S_{z z}(q, \omega)$ of the $X Y$ model at $T=0$ and $h=0 \mathrm{Eq}$. (1.35) by using the density of states of the relevant excitations (1.34) as calculated in the Bethe formalism and the corresponding matrix elements (1.29) as obtained by finite-chain calculations. This approach is readily generalized in order to obtain $S_{z z}(q, \omega)$ for nonzero magnetic field exactly. ${ }^{28}$ A detailed discussion will be published in connection with a study on the anisotropic HB $X Y$ chain. Here, we give the result for the $T=0$ time-dependent two-spin correlation function as calculated by Niemeijer in the fermion representation ${ }^{13}$

$$
\left\langle s_{l}^{z}(t) s_{l+R}^{z}\right\rangle=\sigma^{2}+(2 \pi)^{-2} \int_{-\pi(1-\sigma)}^{+\pi(1-\sigma)} d \psi \int_{-\pi \sigma}^{+\pi \sigma} d \psi^{\prime} \exp \left[i R\left(\psi+\psi^{\prime}-\pi\right)\right] \exp \left[i t\left(\cos \psi-\cos \psi^{\prime}\right)\right]
$$

where

$$
\sigma \equiv N^{-1} \sum_{l=1}^{N}\left\langle s_{l}^{z}\right\rangle=\frac{1}{\pi} \arcsin h, \quad 0 \leqslant \sigma \leqslant \frac{1}{2}
$$

is the magnetization. The structure function is found immediately by Fourier transform, yielding

$$
S_{z z}(q, \omega)=2 \pi \sigma^{2} \delta(\omega) \delta_{q, 0}+\left\{\begin{array}{l}
\frac{\Theta\left(\epsilon_{2 U}(q)-\omega\right) \Theta\left(\omega-\epsilon_{2 L}(q)\right)}{\left[4 J^{2} \sin ^{2}(q / 2)-\omega^{2}\right]^{1 / 2}}, 0 \leqslant q \leqslant \pi \sigma \\
\frac{\Theta\left(\epsilon_{1 U}(q)-\omega\right)\left[\Theta\left(\omega-\epsilon_{2 L}(q)\right)+\Theta\left(\omega-\epsilon_{1 L}(q)\right)\right]}{\left[4 J^{2} \sin ^{2}(q / 2)-\omega^{2}\right]^{1 / 2}}, \quad \pi \sigma \leqslant q \leqslant \pi,
\end{array}\right.
$$

where

$$
\begin{aligned}
& \epsilon_{I L}(q)=2 J \sin \frac{q}{2} \cos \left(\frac{q}{2}-\pi \sigma\right), \\
& \epsilon_{1 U}(q)=2 J \sin \frac{q}{2}, \\
& \epsilon_{2 L}(q)=2 J\left|\sin \frac{q}{2} \cos \left(\frac{q}{2}+\pi \sigma\right)\right|, \\
& \epsilon_{2 U}(q)=\epsilon_{1 L}(q) .
\end{aligned}
$$

As is sketched in Fig. 11, $S_{z z}(q, \omega)$ is governed by two continua of excitations. We shall denote the upper continuum as (1) and the lower continuum as (2). The upper continuum is confined to wave numbers $q \geqslant 2 \pi \sigma$ and lies between the boundaries $\epsilon_{1 L}(q)$ and $\epsilon_{1 U}(q)$ whereas the lower continuum with boundaries $\epsilon_{2 L}(q)$ and $\epsilon_{2 U}(q)$ extends over the whole Brillouin zone. For $q \geqslant 2 \pi \sigma, \epsilon_{2 U}(q)$ coincides with $\epsilon_{1 L}(q)$. The lower continuum has zerofrequency modes, at $q=0$ and $q_{p} \equiv \pi(1-2 \sigma)$, respectively. In the limit $h \rightarrow 0$, the lower continuum vanishes and the upper continuum exhausts all the spectral weight as described in Sec. ID. As the field is increased the "soft mode" at $q=q_{p}$ moves away from $q=\pi$ and reaches the zone center $(q=0)$ at the critical field $h=h_{c}=1$. At the same time the upper continuum collapses into a single excitation at $q=\pi, \omega=2 J$, and the lower continuum reduces to a single branch, both with negligible spectral weight. At $h \geqslant h_{c}$ the entire spectral weight of $S_{z z}(q, \omega)$ is contained in its static part $2 \pi \sigma^{2} \delta(\omega)$. The gap at $q=\pi$ is proportional to the magnetic field

$$
\epsilon_{2 L}(\pi)=2 J \sin \pi \sigma=h
$$




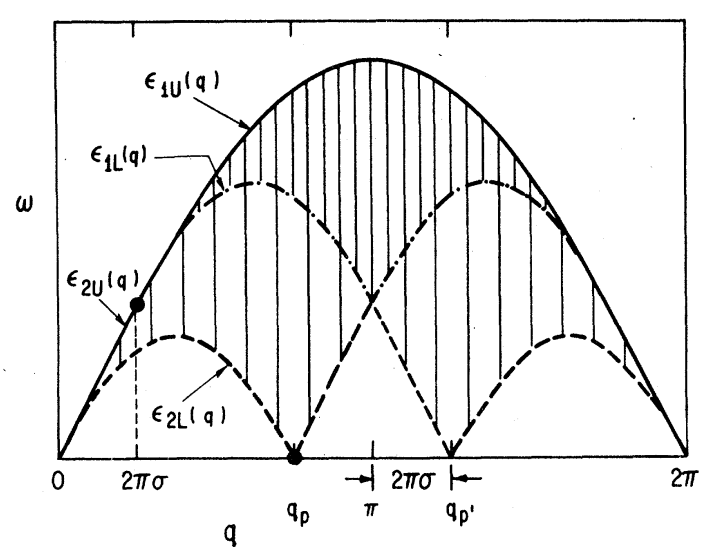

FIG. 11. Sketch of the two continua of excitations which govern the structure function $S_{z z}(q, \omega)$ of the $X Y$ model. All symbols are explained in the text. The center of the Brillouin zone is at $q=0$ and the zone boundary at $q=\pi$.

Figure 12 shows expression (2.3) plotted in relation to the boundaries of the lower and upper continuum for various fixed values of $q$ and $\sigma=0.2$. For small wave numbers, only the lower continuum contributes. $S_{z z}(q, \omega)$ is finite but has a sharp peak at the upper boundary $\epsilon_{2 U}(q)$ and develops a shoulder

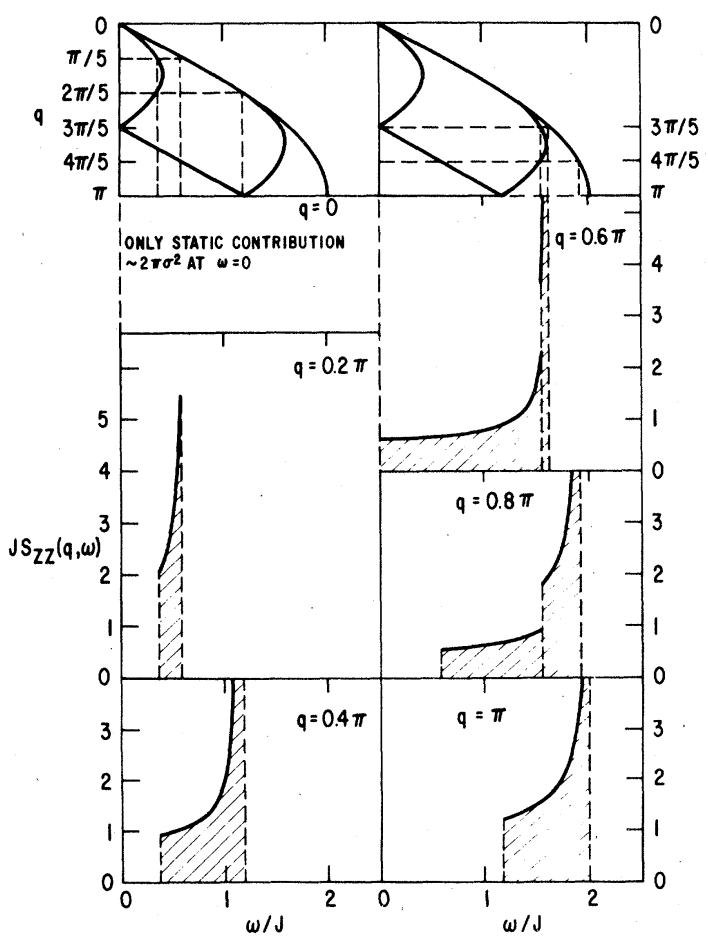

FIG. 12. $S_{z z}(q, \omega)$ for the $X Y$ model at $T=0$ and $\sigma$ $=0.2(h=0.588)$ plotted as a function of $\omega$ for fixed $q=(2 \pi / 10) n, n=0, \therefore \ldots, 5$. The top of the figure shows the boundaries of the two continua contributing to $S_{z z}(q, \omega)$. For $q=0$ there is no spectral weight except the static contribution $2 \pi \sigma^{2} \delta(\omega)$ not shown in the figure. as $q$ increases. For $q>2 \pi \sigma$ both continua contribute. $S_{z z}(q, \omega)$ now has a square-root divergence at the high-frequency cutoff $\epsilon_{1 U}(q)$, and a step both at the middle boundary $\epsilon_{2 L}(q)=\epsilon_{2 U}(q)$ and the lowest boundary $\epsilon_{2 L}(q)$.

\section{Spin-wave continua of the HB AF in applied field}

In this subsection we extend our study of the excitations of the HB AF and their relevance for the zero-temperature dynamics to the case of a nonzero applied field. For $h \neq 0$, we have to treat the longitudinal fluctuations described by $S_{z z}(q, \omega)$ and the transverse fluctuations described by $S_{x x}(q, \omega)$ $\equiv S_{v v}(q, \omega)$ separately. In order to deal more easily with the selection rules (1.13) we decompose the transverse component into two parts according to (1.12):

$$
S_{x x}(q, \omega) \equiv \frac{1}{4}\left[S_{-+}(q, \omega)+S_{+-}(q, \omega)\right] .
$$

A preliminary impression of the results may be gained by recalling the selection rules (1.13). At $T=0, S_{\mu \mu}(q, \omega)$ can still be written in the form (1.14). However, for $h \neq 0$ the ground state $|G\rangle$ is no longer a singlet $\left(s^{T}=0\right)$. The effect of the Zeeman term in the Hamiltonian (1.4) is to progressively depress states of larger total spin $s^{T}$ so that the ground state becomes successively a state belonging to a triplet $\left|s^{T}=1, s_{z}^{T}=1\right\rangle$ to a quintet $\mid s^{T}=2$, $\left.s_{z}^{T}=2\right\rangle$, etc. For fields higher than the critical field ( $h>h_{c}=2$ ) the ground state is ferromagnetic, i.e., it has all spins lined up parallel to the field. Thus, the ground state $|G\rangle$ at arbitrary $h \geqslant 0$ is identified as the lowest state with quantum numbers $s^{T}=[N \sigma(h)]$, where [ ] are Gaussian brackets, $s_{z}^{T}=s^{T}$, where $\sigma=s^{T} / N$ is obviously the $T=0 \mathrm{mag}$ netization. An impression of the behavior of the various eigenstates with field is given by a preliminary study of Bonner and Nagle. ${ }^{61}$ From an examination of the selection rules (1.13a) and (1.13c) we observe the possibility of six different classes of states contributing to $S_{\mu \mu}(q ; \omega)$ at $T=0$. According to Eq. (1.15) $S_{z z}(q, \omega)$ involves matrix elements of the type $\left\langle G\left|s^{z}(q)\right| \lambda\right\rangle$. Therefore, the only excited states which can give nonvanishing contributions must belong to either of the two classes (i) $\left\{\mid s^{T}=N \sigma+1\right.$, $\left.\left.s_{z}^{T}=N \sigma\right\rangle\right\}$ or (ii) $\left\{\left|s^{T}=N \sigma, s_{z}^{T}=N \sigma\right\rangle\right\}$. Note that $|G\rangle$ itself belongs to class (ii). Specifically, if $|G\rangle$ is a triplet state $\left|s^{T}=1, s_{z}^{T}=1\right\rangle$, all excited states $|\lambda\rangle$ with nonvanishing matrix elements $\left\langle G\left|s^{z}(q)\right| \lambda\right\rangle$ are either also triplet states (with maximum $s_{z}^{T}$ ) or quintet states $\left|s^{T}=2, s_{z}^{T}=1\right\rangle$. For a quintet ground state $\left|s^{T}=2, s_{z}^{T}=2\right\rangle$ the contributing excitations are either quintet states also or septet states $\mid s^{T}=3$, $\left.s_{z}^{T}=2\right\rangle$, etc. $S_{-+}(q, \omega)$, on the other hand, involves, according to Eq. (1.8), matrix elements of the type 
$\left\langle G\left|s^{-}(q)\right| \lambda\right\rangle$, and $S_{+-}(q, \omega)$ contains matrix elements of the type $\left\langle G\left|s^{+}(q)\right| \lambda\right\rangle$. Thus, the selection rules (1.13) tell us that the excitations which can contribute to $S_{-+}(q, \omega)$ must belong to the class (iii) $\left\{\left|s^{T}=N \sigma+1, s_{z}^{T}=N \sigma+1\right\rangle\right\}$, and those which can contribute to $S_{+-}(q, \omega)$ must belong to one of the three classes, (iv) $\left\{\left|s^{T}=N \sigma+1, s_{z}^{T}=N \sigma-1\right\rangle\right\},(\mathrm{v})$ $\left\{\left|s^{T}=N \sigma, s_{z}^{T}=N \sigma-1\right\rangle\right\}$, or (vi) $\left\{\mid s^{T}=N \sigma-1\right.$, $\left.\left.s_{z}^{T}=N \sigma-1\right\rangle\right\}$. This diversity, which is summarized in the first three columns of Table II, introduces considerable complexity into our discussion of the $h \neq 0$ dynamics. In the limit $h=0$, where the ground state is a singlet $\left(s^{T}=0\right)$, this complexity is reduced because class (ii) is then excluded by selection rule $(1.13 \mathrm{c})$, and the classes (v) and (vi) no longer exist, leaving the class (i) states $\left\{\left|s^{T}=1, s_{z}^{T}=0\right\rangle\right\}$ as the only potential contributors to $S_{z z}(q, \omega)$ and the states $\left\{\left|s^{T}=1, s_{z}^{T}= \pm 1\right\rangle\right\}$ of classes (iii) and (iv) as the only candidates for $S_{x x}(q, \omega)$, yielding identical results $S_{x x}(q, \omega) \equiv S_{z z}(q, \omega)$ as must be the case for symmetry reasons. In the opposite limit $h=h_{c}$, the ground state has quantum numbers $s^{T}=s_{z}^{T}=N / 2$ and the classes (i), (iii), and (iv) are nonexistent. Thus, the only contribution to $S_{z z}(q, \omega)$ comes from class (ii), which in this limit contains only the ground state. $S_{-+}(q, \omega)$ vanishes in this limit whereas the classes (v) and (vi) can still contribute to $S_{+-}(q, \omega)$, as discussed in detail below.

Our task now is to select among the six classes of states those excitations which are expected to give significant contributions to either component of the structure function. Having identified such states we shall calculate their energies and shall study their spectral weights in order to be able to make specific predictions for possible experiments and to give an explanation for the few experimental results which are already available. This approach has been very successful and promising for the case $h=0$ discussed extensively in Sec. I. There, we have found that the class (i) of triplet states, which give the only contributions to $S_{z z}(q, \omega)$ at $h=0$, contain a subclass of special states which can be identified in the framework of Bethe's formalism. We have shown that they form a two-parameter continuum in $(q, \omega)$ space (called SWC) and that they exhaust almost all the spectral weight of $S_{z z}(q, \omega)$. Such an approach is much more difficult in the nonzero field case and the results will correspondingly be limited. Encouraged by the $h=0$ results, we can speculate that equivalent subclasses of SWC states which appear for $h=0$ in all six classes of Table II also play a major role in the $T=0$ structure function. We shall, therefore, first identify the SWC states for $h \neq 0$ in the Bethe formalism and calculate their energies for the infinite system $(N=\infty)$. In the next subsection, we shall then study their role in the spectral weight distribution of $S_{\mu \nu}(q, \omega)$. It is useful to review here briefly the classification of states of a given $s^{T}=N / 2-r$ sub- space in the Bethe formalism [Eqs. (1.18)-(1.20)] We may concentrate on the case of minimum total spin $s^{T}=s_{z}^{T}$, since states with $s^{T}=s_{z}^{T}+r_{0}, r_{0}>0$, are obtained simply by adding $r_{0}$ zeros to the set $\left\{\lambda_{j}\right\}$ [see, e.g., Eqs. (1.24a) and (1.24b)]. Of special importance for the HB AF is the class $C$ of "spin-wave states" described by $r$ real wave numbers $k_{j}$, which are described by sets $\left\{\lambda_{j}\right\}$ satisfying $1 \leqslant \lambda_{1} \leqslant \lambda_{2} \cdots$ $\leqslant \lambda_{r} \leqslant N-1, \lambda_{j+1}-\lambda_{j} \geqslant 2$, of which there are altogether

$$
g_{N}(r)=\left(\begin{array}{c}
N-r \\
r
\end{array}\right) .
$$

The remaining

$$
\left(\begin{array}{c}
N \\
r
\end{array}\right)-\left(\begin{array}{c}
N \\
r-1
\end{array}\right)-\left(\begin{array}{c}
N-r \\
r
\end{array}\right)
$$

states of the $s^{T}=s_{z}^{T}=N / 2-r$ subspace contain one or more bound spin complexes with complex wave numbers (except for small wave numbers $k \leq 1 / \sqrt{N}$ where these states may also have real wave numbers ${ }^{9}$ ).

Let us first concentrate on the longitudinal excitations. As in the case of $h=0$ (see Sec. ID) we can identify a number of excitations out of class (i) and class (ii) which appear in finite-chain results for $S_{z z}(q, \omega)$ to be "class $C$ " states in Bethe's formalism. These states can be traced back to the $X Y$ model, where they correspond to those excitations which-in the thermodynamic limit-form the two continua of excitations sketched in Fig. 11. As discussed in the preceding subsection these two continua entirely govern $S_{z z}(q, \omega)$ of the $X Y$ model. In order to calculate the energies of these states in the HB AF we have to determine their configurations in the $\lambda$ space of Bethe's ansatz. The prescription for the ground state, which, for a given $h$, has total spin $s^{T}=N / 2-r$ corresponding to a magnetization $\sigma=s^{T} / N$, has changed from Eq. (1.25) for $\sigma=0$ to (assuming $N$ even), ${ }^{24}$

$$
\left\{\lambda_{j}\right\}=\{N \sigma+1, N \sigma+3, \ldots, N-N \sigma-1\} .
$$

The special states out of class (i) (in the following called SWC 1 states) with quantum number $s^{T}=N \sigma+1$ are identified as "hole excitations" in $\lambda$ space, i.e., they are systematically generated by distributing $N / 2-N \sigma-1$ integers $\lambda_{j}$ in accordance with the restrictions

$$
N \sigma+1 \leqslant \lambda_{j} \leqslant N-N \sigma-1
$$

and

$$
\lambda_{j+1}-\lambda_{j} \geqslant 2 \text {. }
$$

These states correspond to the triplet SWC states in the zero-field case. Again the rules (2.11) generally allow for a distribution of the $\lambda_{j}$ 's into three clusters, 
$\stackrel{9}{5}$

율

$\checkmark$ 记

สิ

० 0 ठ

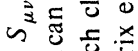

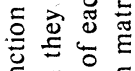

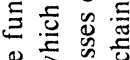

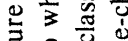

政을

के 3 .

必岀

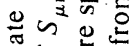

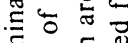

응

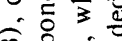

产㣢要

¿ ¿

创号号

혼듀

要造

的政

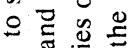

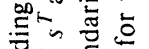

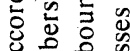

o ह

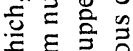

旅

它

픙 눙응

政

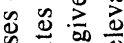

类

००

के

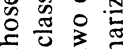

등 $\bar{x}$

\%

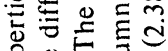

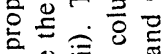

䎸要

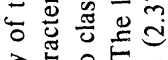

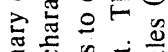

ह

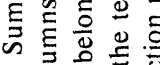

$=\overline{8} \mathrm{Q} \equiv$

पा क क ज

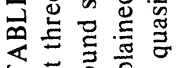

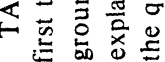
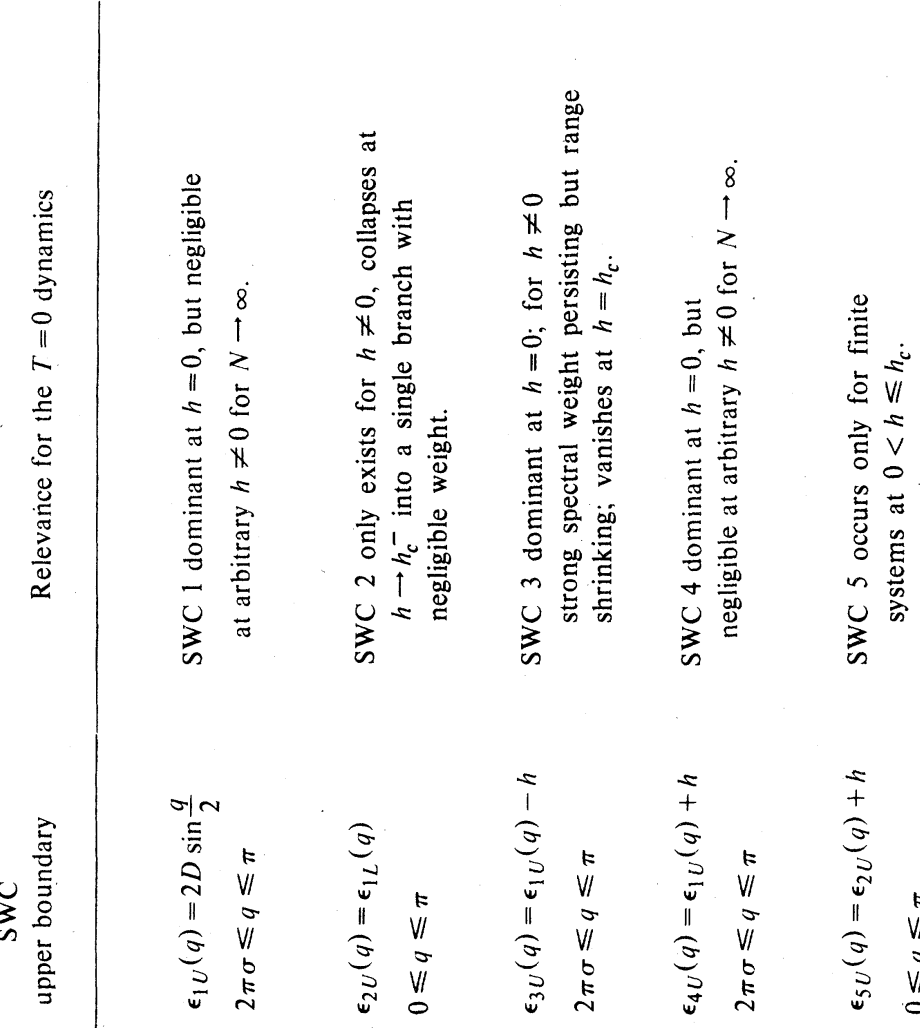

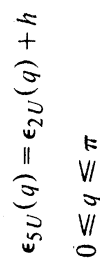
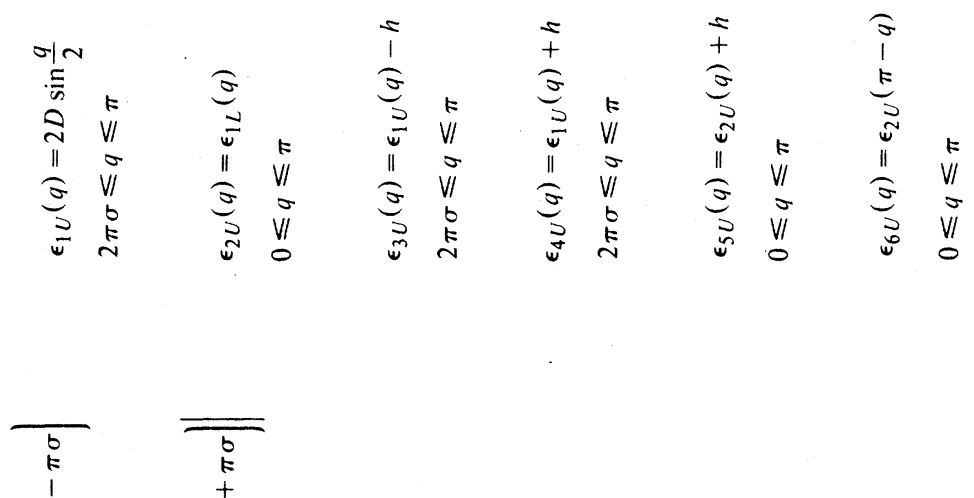

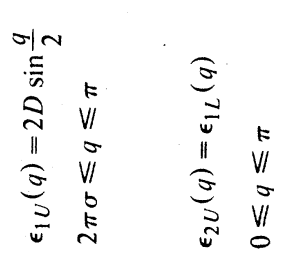

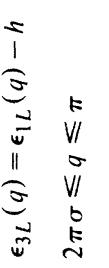

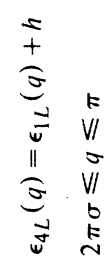

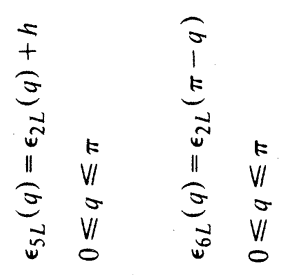

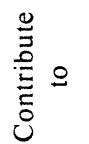

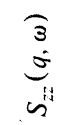

$\underbrace{3}_{\substack{3 \\ 3}}$

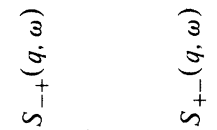

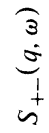

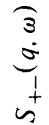

焉总

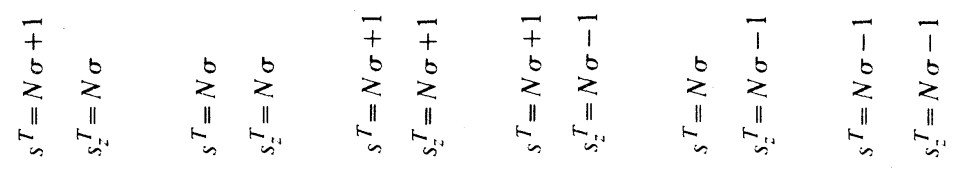

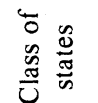

$\fallingdotseq$

3

(
@

$\Xi$

3

$\widehat{3}$ 
producing the same two quantum numbers $q$ (wave number) with respect to the ground state and $q_{m}$ (branch) as for the case $h=0$. In addition to them a quantum number $q_{s}$ related to the magnetization through

$$
q_{s}=2 \pi \sigma
$$

is needed in order to characterize the eigenstate under consideration, i.e., its $\lambda_{j}$ configuration. The special states out of class (ii) (called SWC 2 states), which have the same quantum number $s^{T}=N \sigma$ as the ground state, are identified as "particle-hole excitations" in $\lambda$ space; i.e., they are systematically generated by distributing $N / 2-N \sigma-1$ integers in accordance with the restrictions

$N \sigma+2 \leqslant \lambda_{j} \leqslant N-N \sigma-1, \quad \lambda_{j+1}-\lambda_{j} \geqslant 2$

plus an additional $\lambda_{0}$ in the range

$$
1 \leqslant \lambda_{0} \leqslant N \sigma,
$$

and a corresponding set obtained by reflection at $\lambda=N / 2$. In the zero-field limit $(\sigma=0) \mathrm{Eq}$. (1.25) is the only possible configuration satisfying Eqs. (2.13) and (2.14). It represents the singlet ground state (see Fig. 1). For $\sigma>0$ the rules (2.13) generally allow for a distribution of the $\lambda_{j}$ 's into two clusters, and the isolated $\lambda_{0}$ can be considered as a third cluster. Thus, again we are able to characterize each of these special class (ii) states by the three quantum numbers $q, q_{m}, q_{s}$.

In Appendix A we present an exact calculation of the excitation energies of both SWC 1 and SWC 2 . The evaluation of the resulting expressions depends on the solution of an integral equation. A rigorous solution of this integral equation has been found only in the two limits $h=0$ and $h=h_{c}=2$. Therefore, we present in Appendix B an approximate calculation for intermediate fields, the results of which are now discussed. SWC 1 contains excitations with energies (for $q \geqslant 0$ )

$$
\omega_{1 m}(q)=2 D(h) \sin \frac{q}{2} \cos \left(\frac{q}{2}-\frac{q_{s}}{2}-\frac{q_{m}}{2}\right)
$$

and quantum numbers $q$ (wave number), $q_{m}$ (branch), and $q_{s}$ (magnetization) satisfying the rules

$$
q_{s}=2 \pi \sigma, \quad q_{s} \leqslant q \leqslant \pi, \quad 0 \leqslant q_{m} \leqslant q-q_{s} .
$$

SWC 1 is confined to wave numbers $q \geqslant 2 \pi \sigma$ between the two boundaries $\left(q_{s} \leqslant q \leqslant \omega\right)$

$$
\begin{aligned}
& \epsilon_{1 L}(q)=2 D(h) \sin \frac{q}{2} \cos \left(\frac{q}{2}-\pi \sigma(h)\right), \\
& \epsilon_{1 U}(q)=2 D(h) \sin \frac{q}{2} .
\end{aligned}
$$

This is sketched in Fig. 11, which was originally intended for the $X Y$ model but which qualitatively applies also to the HB AF. SWC 2, on the other hand, contains excitations with energies (for $q \geqslant 0$ )

$\omega_{2 m}(q)=2 D(h) \sin \frac{q}{2} \cos \left(\frac{q}{2}+\frac{q_{s}}{2}-\frac{q_{m}}{2}\right)$,

and quantum numbers $q, q_{m}$, and $q_{s}$ obeying the rules

$$
q_{s}=2 \pi \sigma, \quad 0 \leqslant q_{m} \leqslant q_{s}, \quad q_{m} \leqslant q \leqslant \pi-q_{s}+q_{m} .
$$

This continuum, which is also sketched in Fig. 11, extends over the whole Brillouin zone and is bounded by the two dispersion curves $(0 \leqslant q \leqslant \pi)$

$$
\begin{aligned}
& \epsilon_{2 L}(q)=2 D(h)\left|\sin \frac{q}{2} \cos \left(\frac{q}{2}+\pi \sigma(h)\right)\right|, \\
& \epsilon_{2 U}(q)=\epsilon_{1 L}(q) .
\end{aligned}
$$

All SWC boundaries are listed in columns 4 and 5 of Table II. The prefactor $D$ in all these dispersion relations depends on the field according to

$$
D(h)=\frac{\pi}{2}+\frac{h}{2}\left(1-\frac{\pi}{2}\right) \text {. }
$$

A further result provided by our approximate calculation of Appendix B is an analytic expression for the $T=0$ magnetization curve

$$
\sigma(h)=\frac{1}{\pi} \arcsin \frac{1}{1-\pi / 2+\pi / h}, \quad 0 \leqslant h \leqslant 2 .
$$

The uniform susceptibility $\chi_{z z}(h)$ which is given by the derivative of Eq. (2.24) with respect to $h$, is finite at zero field and diverges as $h$ approaches the critical field $h_{c}=2$. We obtain in the limits $h=0$ and $h_{c}^{-}$

$$
\begin{aligned}
& \chi_{z z}(0)=\frac{1}{\pi^{2}}, \\
& \chi_{z z}(h) \propto \sqrt{h_{c}-h} .
\end{aligned}
$$

respectively, in accordance with the exact results of Griffiths $^{24}$ and Yang and Yang. ${ }^{32}$

The magnetization curve $e^{24}$ is shown as the dashed line in Fig. 13. It is in excellent agreement with the result of Griffiths ${ }^{24}$ (shown as solid line) obtained by numerical solution of an exact integral equation (solid line). Equation (2.24) is эppreciably superior to the Bulaevskii approximation ${ }^{18}$ of the same quantity (not shown in Fig. 13) which is given by the relation

$$
h=\left(1+\frac{2}{\pi} \cos \pi \sigma\right) \sin \pi \sigma+2 \sigma .
$$

The latter does not reproduce the correct zero-field uniform susceptibility. ${ }^{24}$ Very good agreement is obtained also with $(N=10)$ finite-chain results ${ }^{34}$ 


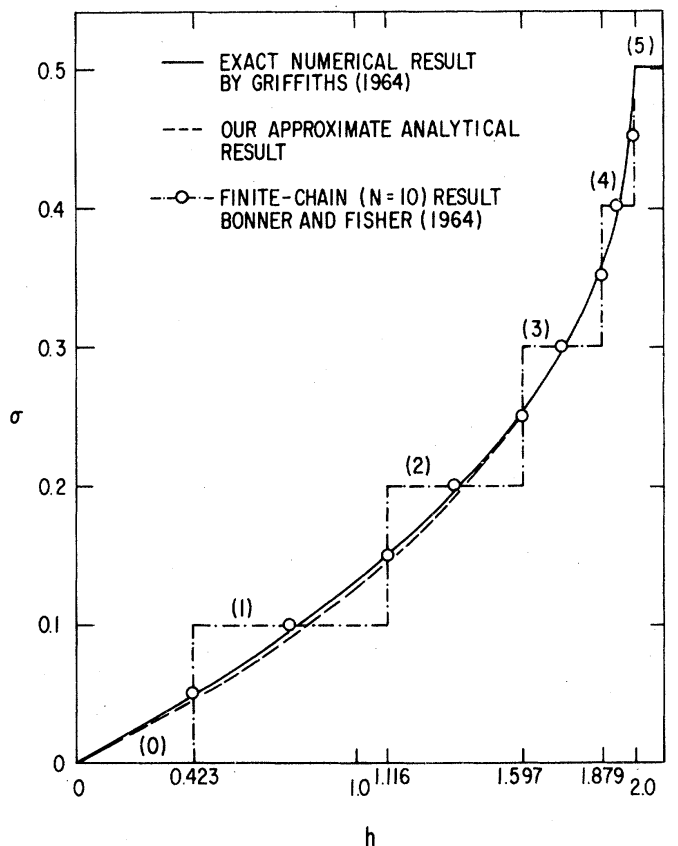

FIG. 13. $T=0$ magnetization curve for the HB AF. The solid curve denotes the exact result (Griffiths) and the dashed curve our analytic approximate result (2.23). The step function represents the $(N=10)$ finite-chain result as described in the text. At the bottom of the figure we give the special values of the field where the ground state changes character. The number in brackets at each horizontal portion is the total spin $s^{T}=[N \sigma]$ of the corresponding ground state.

represented as open circles in Fig. 13. These results are based on the midpoints of the horizontal and vertical portions of the $T=0$ magnetization $\sigma(h)$, which for a finite system is a step function. Such a discontinuous dependence of $\sigma$ on $h$ is caused by the fact that at each vertical step a level of total spin $s^{T}+1$ crosses the previous ground state with total spin $s^{T}$, on account of the Zeeman energy, and becomes the new ground state of the system. At each step, therefore, the character of the field-dependent excitations changes, as also do the corresponding spectral weights. Of course, in the thermodynamic limit, the magnetization steps shrink and their number increases, thus giving rise to a smooth curve. The character of the field-dependent excitations likewise changes continuously with increasing field until the critical field is reached. For fields above the critical field ( $h \geqslant h_{c}=2$ ) the magnetization is saturated, i.e., the ground state is ferromagnetic with all spins aligned parallel to the field. Here, the $T=0$ statics and dynamics are trivially known. ${ }^{62.50}$

Now let us study the class (iii) of transverse excitations. Here, we can identify those excitations which appear in finite-chain results for $S_{-+}(q, \omega)$ with large spectral weight again as "class $C$ " states and, more- over, as states belonging to the same multiplet as the SWC 1 states of class (i). Consequently they form a two-parameter continuum (called SWC 3) which has the same shape as SWC 1 but which is depressed in energy by an amount $h$ with respect to SWC 1 owing to the different Zeeman energy. SWC 1 states and SWC 3 states differ in the Bethe formalism only by different numbers of zeros appearing in the sets $\left\{\lambda_{j}\right\}$ (see Sec. ID). The boundaries of SWC 3 are included in Table II.

Also the remaining classes (iv), (v), and (vi) contain "class $C$ " states. By inspection of the quantum numbers in Table II and by studying the excitations which appear in finite-chain results for $S_{+-}(q, \omega)$, we find that there are SWC states from class (iv) which belong to the same multiplets as the SWC 1 states and the SWC 3 states. Hence, they form another two-parameter continuum (called SWC 4) of the same shape as SWC 1 (and SWC 3), but this time raised in energy by an amount $h$ with respect to SWC 1 owing to the Zeeman splitting. Table II gives the boundaries of SWC 4. Further, there are SWC states out of class $(\mathrm{v})$, which belong to the same multiplet as the SWC 2 states. Obviously, they form in the thermodynamic limit a two-parameter continuum (called SWC 5) which is, due to the Zeeman splitting, raised in energy by an amount $h$ with respect to SWC 2. Its boundaries are also to be found in Table II. Finally, class (vi) also contains SWC states which are the same as the SWC 2 states appearing in $S_{z z}(q, \omega)$ at a slightly lower field (when the ground state has total spin $\left.s^{T}=[N \sigma-1]\right)$, and which belong to the same multiplet as the SWC 4 states appearing in $S_{+-}(q, \omega)$ at a slightly higher field (when the ground state has total spin $\left.s^{T}=[N \sigma+1]\right)$. Hence, these states form, in the thermodynamic limit, a further two-parameter continuum (called SWC 6). Their energies are the same as those of the SWC 2 states and are depressed by an amount $h$ with respect to the SWC 5 states. Note, however, that SWC 2 excitations with momentum transfer (relative to the ground state) $q$ appear in SWC 6 at $q^{\prime}=\pi-q$. This is due to the special property of the $s=\frac{1}{2} \mathrm{HB}$ AF that the ground state (for even $N$ ) as it changes in an increasing field from $s^{T}$ to $s^{T}+1$ alternates between a $k=0$ state and a $k=\pi$ state. This is why the lowest SWC branch of the transverse structure function (i.e., the lower boundary of SWC 6) is reversed with respect to $q=\pi / 2$ compared with the lowest SWC branch of the longitudinal structure function (i.e., the lower boundary of SWC 2). All these continuum boundaries are given in Table II.

\section{Selection rules for the "macroscopic system"}

A major result following from the selection rules (1.13) is the identification from among all the eigen- 
states of those six classes of excitations (summarized in Table II) which contribute to either component of the structure function. This information is also contained in Fig. 14 which shows the classification of all eigenstates according to their quantum numbers $s^{T}$ and $s_{z}^{T}$. For fixed $h$ the ground state belongs to the class at the right-hand side of the row with $s^{T}=N \sigma(h)$. Hence, it moves with increasing field from the bottom square along the diagonal to the top right square. The arrows (solid or dashed) indicate the possible contributions to $S_{\mu \nu}(q, \omega)$ from various classes of states according to the selection rules (1.13a) and (1.13c), which are based on the WignerEckart theorem. In addition to these rules the Wigner-Eckart theorem provides relations between matrix elements involving excited states out of different classes in the same row of Fig. 14. For $h \neq 0$ and in the limit $N \rightarrow \infty$ these relations develop into additional selection rules which further reduce the number of classes contributing to $S_{\mu \nu}(q, \omega)$. The usefulness of the Wigner-Eckart theorem lies in the fact that it separates matrix elements of the spin operator $\left\langle G\left|s^{\mu}(q)\right| \lambda\right\rangle$ in a Clebsch-Gordan coefficient and a reduced matrix element. Specifically, we obtain for matrix elements between the ground state $|G\rangle \equiv\left|s^{T}=N \sigma, s_{z}^{T}=N \sigma, \alpha\right\rangle$ and excitations out of the classes (i) to (vi).

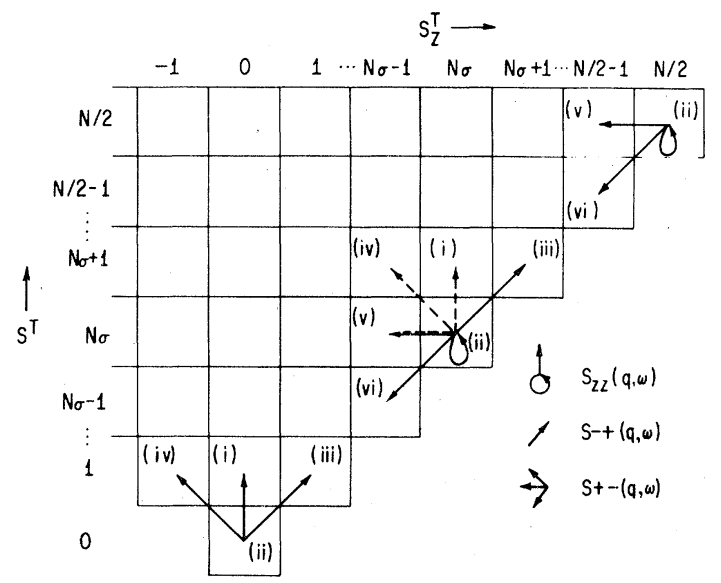

FIG. 14. Rigorous selection rules and selection rules for the macroscopic system. The figure shows a classification of the eigenstates according to their quantum numbers $s^{T}$ and $s_{i}^{T}$. The ground state for fixed $h$ belongs to the class with quantum numbers $s^{T}=s_{z}^{T}=N \sigma(h)$. The arrows (solid or dashed) point to those classes of excitations which are allowed by selection rules (1.13a) and (1.13c) to contribute to either component of $S_{\mu \nu}(q, \omega)$ at $T=0$. The dashed arrows, in particular, point to those classes of excitations which are excluded from contributing to the $T=0$ dynamics by additional selection rules valid for $0<h<h_{c}$ in the limit $N \rightarrow \infty$.

$$
\begin{aligned}
& \text { (i) }\left|\left\langle G\left|s^{z}(q)\right| N \sigma+1, N \sigma, \alpha^{\prime}\right\rangle\right|^{2}=(2 N \sigma+1)\left|\left\langle N \sigma, \alpha\|s(q)\| N \sigma+1, \alpha^{\prime}\right\rangle\right|^{2}, \\
& \text { (ii) }\left|\left\langle G\left|s^{z}(q)\right| N \sigma, N \sigma, \alpha^{\prime}\right\rangle\right|^{2}=N \sigma\left|\left\langle N \sigma, \alpha\|s(q)\| N \sigma, \alpha^{\prime}\right\rangle\right|^{2}, \\
& \text { (iii) }\left|\left\langle G\left|s^{-}(q)\right| N \sigma+1, \alpha^{\prime}\right\rangle\right|^{2}=(2 N \sigma+1)(2 N \sigma+2)\left|\left\langle N \sigma, \alpha\|s(q)\| N \sigma+1, \alpha^{\prime}\right\rangle\right|^{2}, \\
& \text { (iv) }\left|\left\langle G\left|s^{+}(q)\right| N \sigma+1, N \sigma-1, \alpha^{\prime}\right\rangle\right|^{2}=\sqrt{2}\left|\left\langle N \sigma, \alpha\|s(q)\| N \sigma+1, \alpha^{\prime}\right\rangle\right|^{2}, \\
& \text { (v) }\left|\left\langle G\left|s^{+}(q)\right| N \sigma, N \sigma-1, \alpha^{\prime}\right\rangle\right|^{2}=2 N \sigma\left|\left\langle N \sigma, \alpha\|s(q)\| N \sigma, \alpha^{\prime}\right\rangle\right|^{2}, \\
& \text { (vi) }\left|\left\langle G\left|s^{+}(q)\right| N \sigma-1, N \sigma-1, \alpha^{\prime}\right\rangle\right|^{2}=2 N \sigma(2 N \sigma-1)\left|\left\langle N \sigma, \alpha\|s(q)\| N \sigma-1, \alpha^{\prime}\right\rangle\right|^{2},
\end{aligned}
$$

$\alpha$ denotes all additional quantum numbers characterizing a special state. From these equations we immediately deduce the following relations between matrix elements involving excited states which belong to the same multiplet and which therefore appear in different components of $S_{\mu \nu}(q, \omega)$

$$
\begin{aligned}
& \left|\left\langle G\left|s^{z}(q)\right| N \sigma+1, N \sigma, \alpha^{\prime}\right\rangle\right|^{2}=\frac{1}{2(N \sigma+1)}\left|\left\langle G\left|s^{-}(q)\right| N \sigma+1, N \sigma+1, \alpha^{\prime}\right\rangle\right|^{2}, \\
& \left|\left\langle G\left|s^{+}(q)\right| N \sigma+1, N \sigma-1, \alpha^{\prime}\right\rangle\right|^{2}=\frac{1}{(N \sigma+1)(2 N \sigma+1)}\left|\left\langle G\left|s^{-}(q)\right| N \sigma+1, N \sigma+1, \alpha^{\prime}\right\rangle\right|^{2}, \\
& \left|\left\langle G\left|s^{+}(q)\right| N \sigma, N \sigma-1, \alpha^{\prime}\right\rangle\right|^{2}=\frac{2}{N \sigma}\left|\left\langle G\left|s^{z}(q)\right| N \sigma, N \sigma\right\rangle\right|^{2} .
\end{aligned}
$$

For a finite system these relations are useful but their consequence is not very dramatic. For the infinite system, however, they give rise to a singular behavior of $S_{\mu \nu}(q, \omega)$ in the limit $\sigma \rightarrow 0$. Let us first consider relation (2.34). For $\sigma=0$ it confirms that the longitudinal and the transverse structure functions must be equivalent. At fixed $\sigma>0$, on the other hand, the longitudinal excitations out of class (i) are less important than the corresponding transverse excitations out of class (iii) by a factor of order $(N \sigma)^{-1}$. The transverse fluctuations for $\sigma>0$ cannot increase by a factor of order $N \sigma$ with respect to the case $\sigma=0$, because their integrated intensity is limited by sum rule (1.44). The necessary consequence of 
(2.34), therefore, is that the longitudinal excitations of class (i), which dominate $S_{z z}(q, \omega)$ at $h=0$, vanish, in the thermodynamic limit, for any nonzero field. A similar anomaly results from Eq. (2.35) for the transverse excitations of class (iv): They govern $S_{+-}(q, \omega)$ at $h=0$ but they vanish, in the limit $N \rightarrow \infty$, for any nonzero $h$. By the same argument we conclude from Eq. (2.36) that the transverse excitations of class $(v)$ vanish in the thermodynamic limit for fields $0<h \leqslant h_{c}$. An exception is the single transverse excitation of class $(\mathrm{v})$ at $q=0$. Its spectral weight is related by Eq. (2.36) to the static contribution to $S_{z z}(q, \omega)$. Since this static contribution becomes of macroscopic order at $\sigma \neq 0$, it does not suppress the spectral weight of the corresponding transverse excitation at $q=0$. Hence, the dashed arrows in Fig. 14 denote those classes of excitations which only contribute for finite $N$ to the $T=0$ dynamics of the HB AF at nonzero $h$.

In summary, we have found the following selection rules for a macroscopic $\mathrm{HB} \mathrm{AF}$ system at $0<h<h_{\mathrm{c}}$ and $q \neq 0$, in addition to Eq. (1.13)

$$
\begin{gathered}
\left\langle N \sigma, N \sigma\left|s^{z}(q)\right| s^{T^{\prime}}, s_{z}^{T^{\prime}}\right\rangle \rightarrow 0 \\
\text { unless } s^{T^{\prime}}=s_{z}^{T^{\prime}}=N \sigma, \\
\left\langle N \sigma, N \sigma\left|s^{ \pm}(q)\right| s^{T}, s_{z}^{T^{\prime}}\right\rangle \rightarrow 0 \\
\text { unless } s^{T^{\prime}}=s_{z}^{T^{\prime}}=N \sigma \pm 1 .
\end{gathered}
$$

Of course, these rules are only poorly satisfied by finite-chain calculations. However, now that we are aware of them, we can confidently study finite-chain matrix elements in order to gain valuable insight into the spectral weight distribution of $S_{\mu \nu}(q, \omega)$.

\section{E. $S_{z z}(q, \omega)$ of the HB AF at $T=0$ and $h \neq 0$}

In Figs. 15(a), 15(b), 15(c), and 15(d) we show the spectral excitations contributing to $S_{z z}(q, \omega)$ at $T=0$ for a cyclic chain of $N=10$ spins. The four plots belong to field ranges corresponding to the horizontal step portions (1), (2), (3), and (4), respectively, of Fig. 13. The lowest step (0), of course, corresponds to the zero-field case, which has been presented in Fig. 1 and extensively discussed in Sec. IB. The ground state (always drawn at the bottom left) has changed from an $s^{T}=0$ singlet at $h=0$ to a $s^{T}=1$ state (triangle) at $h=0.423$. At higher fields it subsequently changes to an $s^{T}=2$ state (square), an $s^{T}=3$ state (inverted triangle), an $s^{T}=4$ state (diamond) and finally, at the critical field, to the ferromagnetic state with $s^{T}=N / 2=5$. This last field range is not shown in Fig. 15. The solid lines reproduce the boundaries of SWC 1 and SWC 2 as calculated in Sec. II C by use of Bethe's formalism (see also Table II). The finite-chain SWC 1 states are given by the full symbols within the continuum boundaries $\epsilon_{1 L}(q)$ and $\epsilon_{1 U}(q)$. At $h=0$, these states exhaust almost all the spectral weight of $S_{z z}(q, \omega)$ for the finite system as well as for the infinite system, as has been shown in Sec. I. At $h=0$, they are still present in a finite "nonmacroscopic" system, although the SWC 1 is steadily shrinking in range and losing spectral weight. For a macroscopic system, however, we know from Eq. (2.37) that as soon as $h$ becomes nonzero the SWC 1 states [together with all the other states of class (i)] immediately lose their spectral weight in $S_{z z}(q, \omega)$. Thus, we are left with the SWC 2 states including the other non-negligible excitations out of class (ii). The finite-chain SWC 2 excitations including the ground state are shown by the full symbols within the continuum boundaries, $\epsilon_{2 L}(q)$ and $\epsilon_{2 U}(q)$, throughout the sequence of Fig. 15. At $h=0$, this continuum (except for the ground state) is nonexistent. In the field range (1) [Fig. 15(a)] the SWC 2 states form a single branch with spectral weight increasing as $q$ approaches $\pi$. At higher fields [Figs. 15(b) and 15(c)], further SWC 2 branches appear. The matrix elements of these excitations clearly increase towards the lower boundary $\epsilon_{2 L}(q)$, which consists of two different branches, separated by the zero-frequency mode $q_{p}$. This indicates that in the thermodynamic limit, when the states close up as $1 / N$, the spectral weight distribution in $S_{z z}(q, \omega)$ has a divergence or a cusp at the lower threshold $\epsilon_{2 L}(q)$. A more quantitative description of the expected singularities at both lower thresholds requires further investigation. An extension of this approach which uses extrapolations of finite- $N$ matrix elements and expressions for infinite- $N$ densities of states derived in the Bethe formalism is in preparation. Further, $S_{z z}(q, \omega)$ contains a zero-frequency mode with vanishing spectral weight at $q=0$ and one with large spectral weight at $q=q_{p}=2 \pi(1-\sigma)$.

Apart from the SWC 2 states there are other excitations out of class (ii) with non-negligible spectral weight, which we cannot account for in the above prescription. They are drawn as open symbols. We are already familiar with such states outside the SWC for $h=0$, namely, the "triplet sea." At intermediate fields [Figs. 15(a) and 15(b)], most of them appear in the range of the SWC 1 states or somewhat above. However, there seems to be a clear discontinuity in spectral weight between SWC 2 excitations and the other higher lying excitations [see, e.g., Fig. 15(b)]. Therefore, we may speculate that also the upper boundary of the SWC 2 gives rise to some structure in the inelastic neutron scattering cross section. This feature will be further analyzed in Sec. II G. In Fig. 15(c), which corresponds to a field range already close to the critical field, we expect some of the excitations to be identifiable with states close to the ground state of the Heisenberg Ferromagnet (HB 

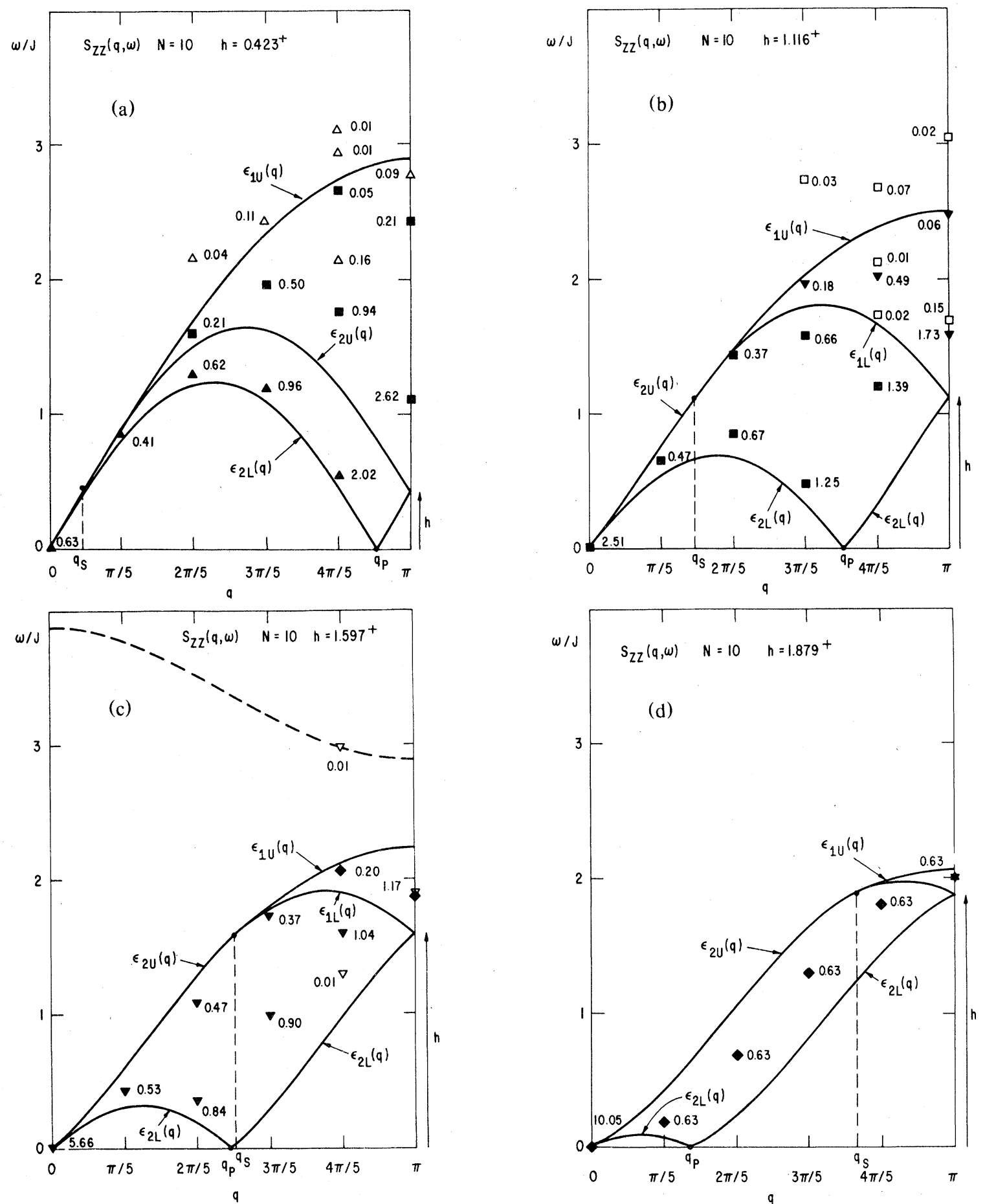

FIG. 15. (a)-(d) Spectral representation of $S_{z z}(q, \omega)$ for the HB AF with $N=10$ at $T=0$ and $h$ in the ranges (1) to (4) of Fig. 13. For each value of the wave number $q=(2 \pi / 10) n, n=0,1, \ldots, 5, S_{z z}(q, \omega)$ is a sum of $\delta$ functions according to Eq. (1.14). In each plot the ground state, whose character changes with field, is drawn at $q=0, \omega=0$. The different symbols indicate the quantum numbers $s^{T}$ of the states: $s^{T}=1$ (triangle), $s^{T}=2$ (square), $s^{T}=3$ (inverted triangle), $s^{T}=4$ (diamond), $s^{T}=5$ (star). Full symbols denote "class $C$ " states and open symbols "anomalous" states as described in the text. The numbers give the corresponding spectral weights $M_{\lambda}^{z}$. Excitations not shown in the figures have spectral weights which are smaller by at least one order of magnitude. The full lines represent the SWC boundaries as given in Table II for the appropriate field indicated at the top of each figure. The dashed line in $\mathrm{c}$ is the dispersion relation of bound spin complexes with two overturned spins (see Ref. 27). 
FM) in zero field. Indeed, the single $s^{T}=3$ excitation near the top of the figure is a spin-wave bound state of two overturned spins. In the finite system there will be a whole branch of such bound states present as indicated by the dashed line. The relevance of the bound states for the AF dynamics near the critical field is not yet clear and needs further investigation. Figure 15(d) shows the situation for the field range (4) of Fig. 13 closest to the critical field. The ground state has $s^{T}=4$ and is identified as a ferromagnetic spin wave of one overturned spin. Together with the states of SWC 2 it forms the complete $(1-\cos q)$-like ferromagnetic spin-wave branch, and the single surviving state in the shrinking SWC 1 with total spin $s^{T}=N S=5$ is one component of the ferromagnetic ground-state multiplet. There are no other excitations which can contribute to $S_{z z}$ in this field range, and the matrix elements of the remaining excitations can be calculated exactly. ${ }^{50}$ They all have the same value $M_{\lambda}^{z}=2 \pi / N$, and will therefore have negligible spectral weight in the thermodynamic limit. In fact, if we examine the states along the lower threshold $\epsilon_{2 L}(q)$ for the sequence of Figs. 15(a) through $15(\mathrm{~d})$, we observe that their spectral weight continuously diminishes as the field increases. Hence we conclude that the relative importance of the longitudinal $S_{z z}$ fluctuations decreases as the critical field is approached. In fact, the spectral weight in $S_{z z}$ is increasingly absorbed by the static part at $q=0, \omega=0$. For fields above the critical field corresponding to the saturation magnetization $\sigma=\frac{1}{2}$ [field range (5)] the ground state is ferromagnetic with all spins aligned parallel to the $z$ axis and the longitudinal fluctuations are completely suppressed; i.e., the static part of $S_{z z}(q, \omega)$ exhausts all the spectral weight. We shall later see that, by contrast, the relative importance of the transverse $S_{x x}$ fluctuations steadily increases with field.

As mentioned before for $0<h<h_{c}$ some "anomalous" states which do not belong to either SWC but have non-negligible spectral weight appear in $S_{z z}(q, \omega)$ in Figs. 15(a) to 15 (c). There is a possibility of describing at least some of these states in the Bethe formalism by slightly changing the restrictions (2.13) and (2.14) of the allowed $\lambda_{j}$ configurations for the SWC 2 states. This would lead, in the thermodynamic limit, to new SW continua. Such new continua could possibly account for most of the "anomalous" states appearing inside or near the boundary of SWC 1. So far, a direct identification with the $N=0$ "anomalous" states has not been possible. Hence, the nature of the "anomalous" states and their relevance for $S_{z z}(q, \omega)$ requires further study.

The field dependence of the HB AF also gives us an interesting insight into the convergence to the thermodynamic limit of finite systems with even and $\operatorname{odd} N$. It may be observed from Fig. 1 that, as is well known, the ground state (drawn at $q=0, \omega=0$ ) for an even- $N$ system at $h=0$ is a singlet $\left(s^{T}=0\right)$. The next higher state is a triplet $\left(s^{T}=1\right)$ at $q=\pi$ with a small energy gap. This gap vanishes in the thermodynamic limit as $1 / N$ in accordance with the $\mathrm{dCP}$ triplet dispersion curve (2.2a) which has a zerofrequency mode at $q=\pi$. The form of the corresponding dispersion curve for odd $N$ is not so familiar. ${ }^{5}$ The ground state at $h=0$ is fourfold degenerate, consisting of two doublet $\left(s^{T}=\frac{1}{2}\right)$ states with wave numbers differing by $(\pi-\pi / N)$. Figure 16 shows $S_{z z}(q, \omega)$ for $N=9$ at $h=0$. The excitation spectrum consists of two continua: a lower continuum of $s^{T}=\frac{1}{2}$ states (SWC 2) and an upper continuum of $s^{T}=\frac{3}{2}$ states (SWC 1). Continuum boundaries are drawn for an effective field $h=0.496$, obtained by locating $q_{p}$ at the position of the zerofrequency excitation. Hence the odd- $N$ chain appears to behave in all respects like an even- $N$ chain in a nonzero magnetic field. For $N \rightarrow \infty$ the dCP dispersion (2.2a) of the lowest branch is regained, but not in terms of a vanishing energy, but rather a vanishing " $q$ deviation" $\Delta q=\pi / N$.

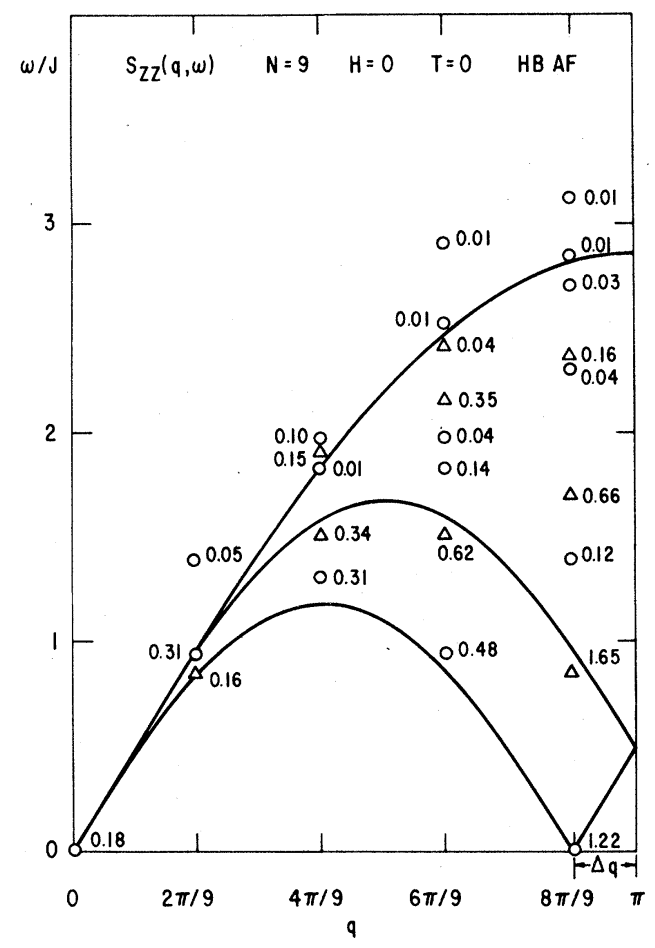

FIG. 16. Spectral representation of $S_{z z}(q, \omega)$ for the HB AF with $N=9$ at $T=0$ and $h=0$. The ground state is fourfold degenerate and consists of two doublets $\left(s^{T}=\frac{1}{2}\right)$ with wave numbers differing by $(\pi-\pi / N)$. Doublet states $\left(s^{T}=\frac{1}{2}\right)$ are denoted by circles and quartet states $\left(s^{T}=\frac{3}{2}\right)$ by triangles. The numbers give the corresponding spectral weights $M_{\lambda}^{z}$. The continuum boundaries are given in Table II for an effective field $h=0.496$. 


\section{F. $S_{-+}(q, \omega)$ and $S_{+-}(q, \omega)$ of the $\mathrm{HB} \mathrm{AF}$ at $T=0$ and $h \neq 0$}

Figures $17(\mathrm{a}), 17(\mathrm{~b})$, and $17(\mathrm{c})$ show the spectral excitations contributing to $S_{-+}(q, \omega)$ at $T=0$ for $N=10$. The applied field is in the same ranges as in Figs. 15(a), 15(b), and 15(c). The $h=0$ case, where we have

$$
S_{z z}(q, \omega)=\frac{1}{2} S_{+-}(q, \omega)=\frac{1}{2} S_{-+}(q, \omega),
$$

is represented in Fig. 1. In accordance with the selection rules (1.13), the finite-chain results show that only class (iii) excitations contribute to $S_{-+}(q, \omega)$. Moreover, they clearly show that the SWC 3 states (shown as full symbols) have dominant spectral weight. The solid lines represent the continuum boundaries $\epsilon_{3 L}(q)$ and $\epsilon_{3 U}(q)$ as given in Table II. The spectral weight strongly increases towards $q=\pi$ and towards the lower continuum boundary $\epsilon_{3 L}(q)$. Therefore, we expect that in the limit $N \rightarrow \infty$, $S_{-+}(q, \omega)$ has a divergence or a cusp at the lower boundary $\epsilon_{3 L}(q)$, which should be experimentally observable. Thus, for $0 \leqslant h \leqslant h_{c}, S_{-+}(q, \omega)$ has a zero-frequency mode with large spectral weight at $q=\pi$ and one with negligible spectral weight at $q=q_{s}=2 \pi \sigma$. For $h \rightarrow h_{c}$, the SWC 3 collapses into a

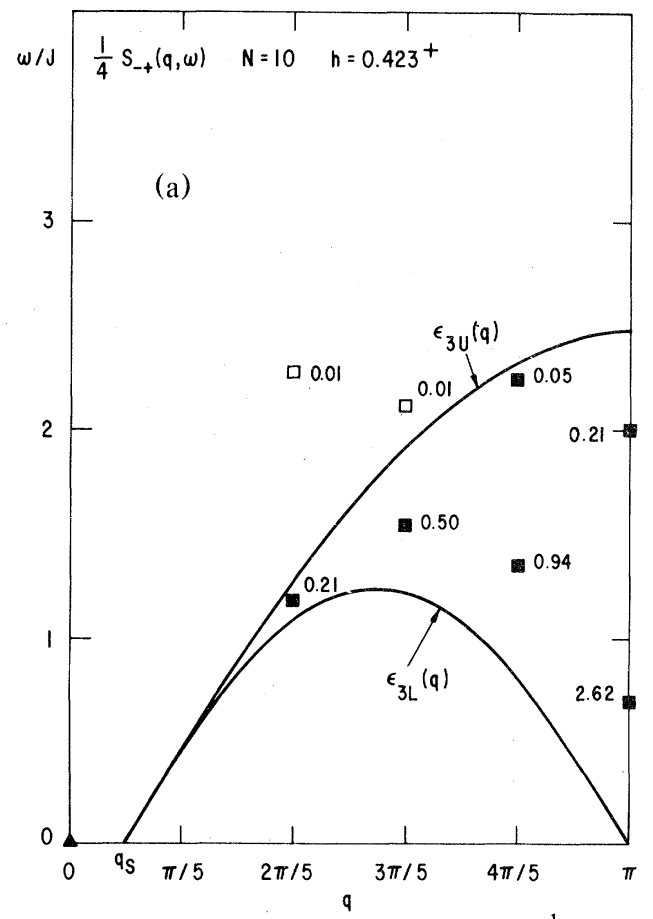

FIG. 17. (a) -(c) Spectral representation of $\frac{1}{4} S_{-+}(q, \omega)$ for the HB AF with $N=10$ at $T=0$ and $h$ in the ranges (1) to (3) of Fig. 13. The ground state which is a singlet $\left(s^{T}=0\right)$ at $h=0$ changes subsequently to an $s^{T}=1$ state (triangle), an $s^{T}=2$ state (square), and an $s^{T}=3$ state (inverted triangle). In each of the three cases the allowed excitations have quantum numbers $s^{T}=2, s^{T}=3$, and $s^{T}=4$ (diamonds), respectively. Full symbols distinguish the SWC 3 excitations. The numbers give the spectral weight $M_{\lambda}^{-}$of each excitation, according to Eq. (1.15). The solid lines represent the SWC 3 boundaries $\epsilon_{3 L}(q)$ and $\epsilon_{3 U}(q)$ for the infinite system as given in Table II. Excitations not shown in the figures have spectral weight which is smaller by at least one order of magnitude. single excitation at $\omega=0$ and $q=\pi$, which then vanishes for $h>h_{c}$.

Note that there are again "anomalous" states apweight. Their role in a macroscopic system is unclear so far and needs further investigation. Note further, that the SWC 3 spectral weight distribution is the same as in SWC 1 of Figs. 15(a), 15(b), and 15(c) up to a factor $2(N \sigma+1)$ enhancing the SWC 3 spectral weight in $S_{-+}(q, \omega)$ with respect to the SWC 1 spectral weight in $S_{z z}(q, \omega)$.

By studying $S_{+-}(q, \omega)$ we are faced with greater complexity, since the selection rules now allow three classes of excitations [(iv), (v), and (vi)] to contribute to the structure function, at least for finite $N$. In Figs. 18(a), 18(b), 18(c), 18(d), and 18(e) we show the spectral excitations contributing to $S_{+-}(q, \omega)$ at $T=0$ for a cyclic chain of $N=10$ spins. The five plots belong to the field ranges corresponding to the horizontal step portions (1) to (5), respectively, of Fig. 13. With increasing field the ground state (at the bottom left in each plot) changes from a singlet ( $s^{T}=0$, shown in Fig. 1 as circle) subsequently to states with increasing total spin, $s^{T}=1$ (triangle), $s^{T}=2$ (square), $s^{T}=3$ (inverted triangle), $s^{T}=4$ (diamond), $s^{T}=N s=5$ (star), until saturation is

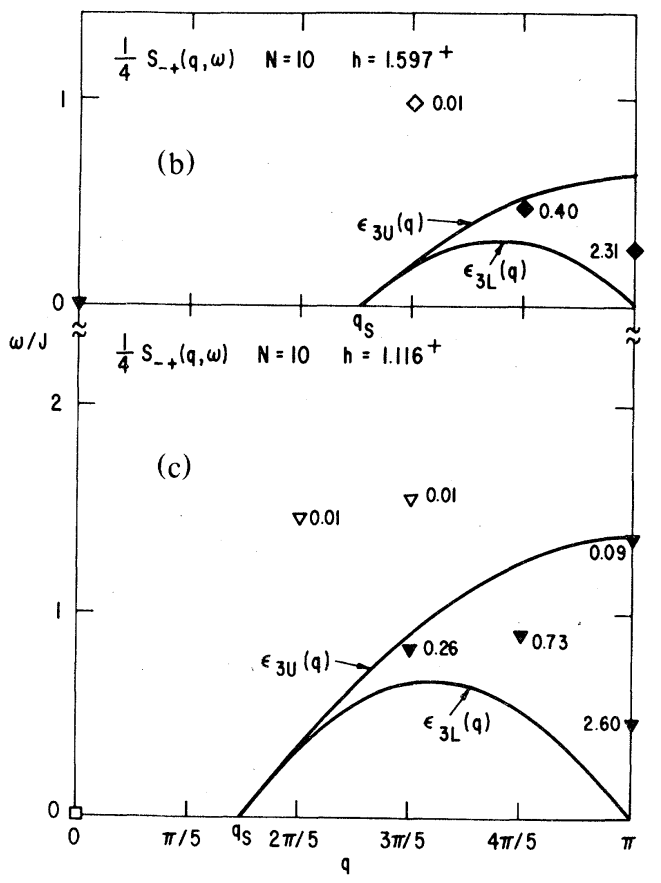
pearing outside SWC 3 with non-negligible spectral 

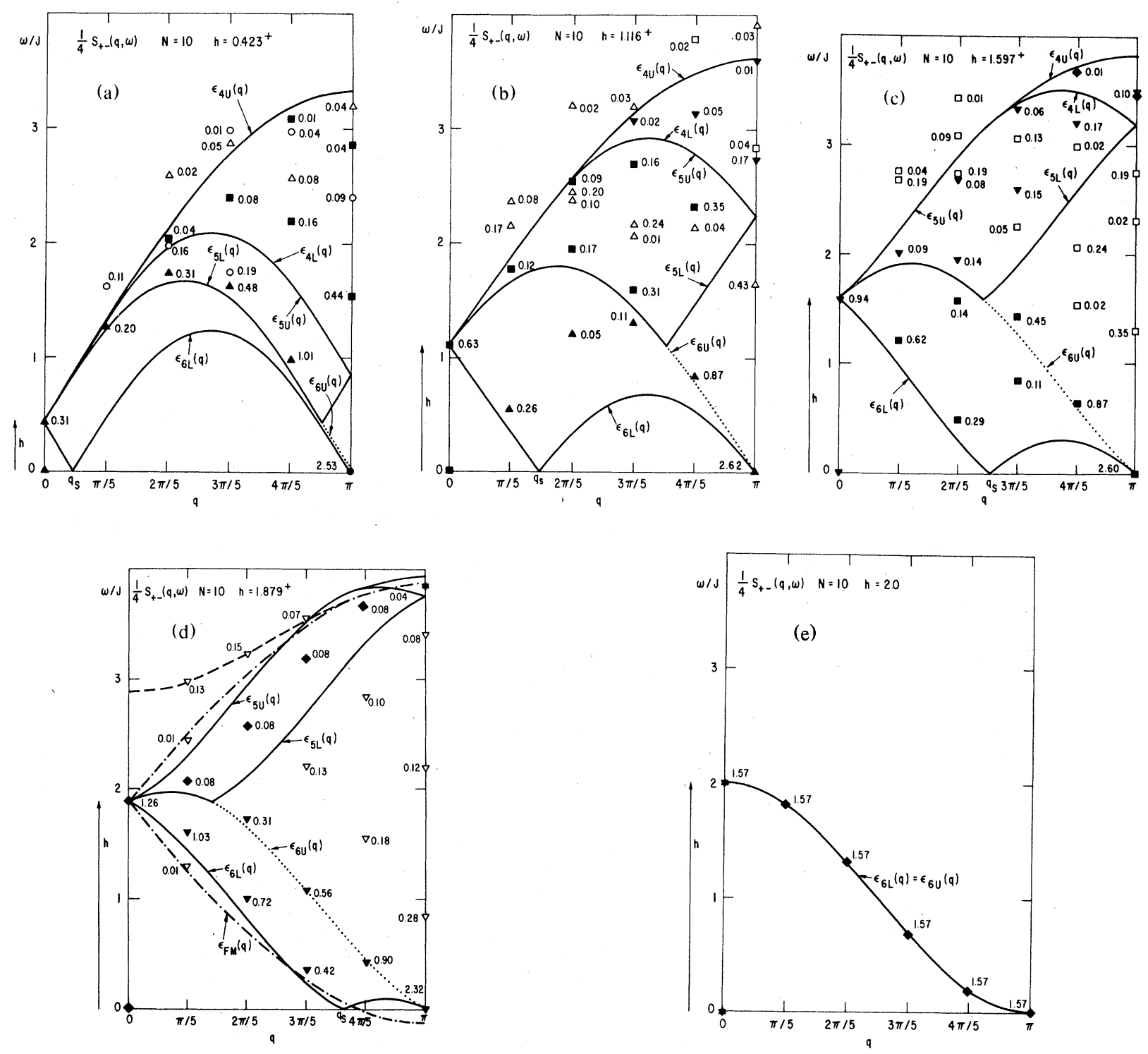

FIG. 18. (a)-(e) Spectral representation of $\frac{1}{4} S_{+-}(q, \omega)$ for the HB AF with $N=10$ and $h$ in the ranges (1) to (5) of Fig. 12. The quantum numbers of the excitations are characterized by different symbols: circles $\left(s^{T}=0\right)$, $\operatorname{triangles}\left(s^{T}=1\right)$, squares $\left(s^{T}=2\right)$, inverted triangles $\left(s^{T}=3\right)$, diamonds $\left(s^{T}=4\right)$, and stars $\left(s^{T}=5\right)$. In each plot the ground state, whose character changes with field, is drawn at $q=0, \omega=0$. Full symbols distinguish the various SWC states. The numbers give the spectral weights $M_{\lambda}^{+}$of each excitation, according to Eq. (1.15). The solid lines represent the various SWC boundaries [except $\epsilon_{6 U}(q)$, which is drawn as dotted line] as given in Table II. In (d), the dashed line denotes the dispersion relation of bound spin complexes with two overturned spins (see Ref. 27) and the dot-dashed lines denote the boundaries of the ferromagnetic two-spinwave continuum (2.39). Excitations not shown in the figures have spectral weight which is smaller by at least one order of magnitude.

reached at the critical field. The finite-chain SWC 4 states are shown as full symbols between the continuum boundaries $\epsilon_{4 L}(q)$ and $\epsilon_{4 U}(q)$ in the sequence of Fig. 18. At $h=0$ (shown in Fig. 1) these states dominate $S_{+-}(q, \omega)$, whereas we know from Eq. (2.38) that for a macroscopic system at any nonzero $h$ they can be neglected in $S_{+-}(q, \omega)$. By the same argument, the SWC 5 states which appear in the finite- chain results for $0<h<h_{c}$ as full symbols between the boundaries $\epsilon_{5 L}(q)$ and $\epsilon_{5 U}(q)$ can be neglected for a macroscopic system (except at $q=0$, as discussed above). The same is true for all the other excitations out of class (iv) or (v) (shown as corresponding open symbols). Hence, we are left with the SWC 6 states including the other important excitations out of class (vi). The SWC 6 excitations 
are given by the full symbols below $\epsilon_{5 L}(q)$ [i.e., roughly between $\epsilon_{6 L}(q)$ and $\left.\epsilon_{6 U}(q)\right]$. At $h=0$ SWC 6 does not exist. For $N=10$, a first complete branch appears at $h=1.116$ and further branches at higher fields. At the critical field SWC 6 collapses into the single "ferromagnetic" spin-wave branch to be discussed below. Thus, we have obtained an inventory of the lower-lying excitations which dominate $S_{+-}(q, \omega)$. However, the intensity pattern of these SWC 6 excitations is more complicated than that in $S_{-+}(q, \omega)$ and $S_{z z}(q, \omega)$. Furthermore, a considerable amount of spectral weight is distributed over higher-lying excitations out of class (vi), again with a complicated intensity pattern. Therefore, our calculations do not enable us to give a detailed prediction of the behavior of $S_{+-}(q, \omega)$ for a macroscopic system. Nevertheless, the finite-chain matrix elements indicate that $S_{+-}(q, \omega)$ has a maximum near the lower boundary $\epsilon_{6 L}(q)$ of SWC $6 . S_{+-}(q, \omega)$ has two zero-frequency modes, one at $q=q_{s}=2 \pi \sigma$ and one at $q=\pi$. The latter has much larger spectral weight. Further, we observe that most of the spectral weight of class (vi) excitations is (roughly) concentrated below the $\epsilon_{3 U}(q)$ boundary (except near the critical field). Thus, we might expect to observe some structure in $S_{+-}(q, \omega)$ at this boundary, too. Further predictions based on the present results would be too risky. In order to give more precise predictions of the behavior of $S_{+-}(q, \omega)$ we need information on the energies of the higher-lying class (vi) excitations.

We have so far not been able to extract this information from the Bethe formalism. For this reason, it is of particular interest to study the situation close to the critical field [Fig. 18(d)], where some exact results are available. It sheds some light on the nature of the states, which contribute to $S_{+-}(q, \omega)$, but which do not belong to the SWC 6 states. The excitations which appear in $S_{+_{-}}(q, \omega)$ at $h=h_{c}^{-}$are a subset of states which characterize the excitations of the 1D $s=\frac{1}{2}$ HB FM at zero field. ${ }^{63}$ As is well known, the excitations with $s_{z}^{T}=N / 2-2$ of the HB FM consist of two classes. One class is the twospin-wave continuum, and the other class consists of bound spin complexes. Now, the excitations of Fig. 18(d) comprise all the states of the ferromagnetic two-spin-wave continuum (the boundaries of which are indicated by dot-dashed lines) and a single branch of bound states (indicated as dashed line). Our lowest SWC boundary $\epsilon_{6 L}(q)$ does not coincide exactly with the corresponding ferromagnetic two-spinwave continuum boundary

$$
\epsilon_{\mathrm{FM}}(q)=2 J(1-\sin q / 2),
$$

but the difference is small. It is interesting to note, however, that, due to this difference, in the limit $h \rightarrow h_{c}^{-}$the dCP spin waves as calculated by Ishimura and $\mathrm{Shiba}^{59}$ for arbitrary $h$ do not correspond to the lowest lying excitations of the HB AF. Nevertheless, the finite-chain matrix elements in Fig. 18(d) indicate that for the spectral weight distribution in $S_{+-}(q, \omega)$, $\epsilon_{6 L}(q)$ serves rather better as the lower boundary than does $\epsilon_{\mathrm{FM}}(q)$. Hence, we now know that the Bethe ansatz class $C$ prescription used extensively in this work does not provide all the spin-wave states (by definition, it does not provide the bound states). We may, therefore, assume that states occurring in $S_{\mu \nu}(q, \omega)$ which are not "class $C$ " states can nevertheless be spin-wave states. We have also learned that at least some of the excitations which appear in $S_{\mu \nu}(q, \omega)$ have bound-state character. Clearly the nature of all the excitations which do not belong to any SWC but which contribute significantly to either component of $S_{\mu \nu}(q, \omega)$ is a very interesting question in need of further investigation.

When the critical field is finally reached, the complexity of the behavior of $S_{+-}(q, \omega)$ is drastically reduced [see Fig. 18(e)]. The ground state has $s^{T}=N / 2$, i.e., all spins are lined up parallel to the field, and the only excitations which can contribute to $S_{+-}(q, \omega)$ are the ferromagnetic spin waves forming a single branch. An exact calculation yields ${ }^{50}$

$$
S_{+-}(q, \omega)=2 \pi \delta(\tilde{\omega}-J(1+\cos q))
$$

in accordance with the finite-chain result for $N=10$. Note the difference in spectral weight between the longitudinal fluctuations at $h=h_{c}$ [Fig. 15(d)] and the transverse fluctuations at $h=h_{c}$ [Fig. 18(e)]. Whereas in the case of $S_{z z}(q, \omega)$, the matrix elements of the states along the single branch have the same constant value $M_{\lambda}^{z}=2 \pi / N$, and hence vanish in the thermodynamic limit, the matrix elements of $S_{+-}(q, \omega)$ have the constant value $M_{\lambda}^{+}=2 \pi$. Hence, as noted in Sec. IIE, it is the transverse dynamical correlation function which dominates near and above the critical field. In fact, the (exactly calculated) behavior of $S_{x x}(q, \omega)$ at $h \geqslant h_{c}$ is identical to that predicted by standard spin-wave theory. Thus, at these values of applied field, no quantum effects are expected.

\section{G. Experimental comparison}

Having described, theoretically, the behavior of the longitudinal and transverse dynamic correlation function $S_{z z}(q, \omega)$ and $S_{x x}(q, \omega)$ of the HB AF in an applied magnetic field, we now discuss possible experimental comparisons. The complex and unusual features we have observed in the preceding discussion, together with the striking differences between the quantum system and the classical $(s=\infty)$ system, suggest that experimental investigation using, for example, neutron scattering, would be of considerable interest. We shall, therefore, discuss features of our results suitable for experimental study, such as 
the relative location of peaks in the scattering intensity (spectral weight distribution) at low temperatures, and the variation of integrated intensity (static correlation function) as a function of $q$, also for low $T$.

The dramatic difference between $S_{x x}(q, \omega)$ and $S_{z z}(q, \omega)$, especially in the vicinity of the critical field, raises the question of the limiting behavior as $h \rightarrow 0$. Since isotropy prevails at $h=0$,

$$
S_{z z}(q, \omega)=\frac{1}{2} S_{+-}(q, \omega)=\frac{1}{2} S_{-+}(q, \omega)
$$

and it is of interest to see how the three components approach the same zero-field structure. Figures 19(a) and 19(b) show, respectively, the important features of $S_{z z}(q, \omega)$ and $S_{x x}(q, \omega)$ for a small reduced field of $h=0.21$, appropriate to $70 \mathrm{kOe}$ neutron scattering experiments on CPC. ${ }^{43}$ From these figures it is clear how the various branches of the $S_{z z}(q, \omega)$ and $S_{x x}(q, \omega)$ converge to the isotropic $h=0$ limit.

Let us first consider the longitudinal correlations. We have observed in Sec. II E that in SWC 2, which dominates $S_{z z}(q, \omega)$ at $T=0$ and $h \neq 0$, the spectral weight increases strongly towards the lower boundary $\epsilon_{2 L}(q)$. Therefore, we expect to see a distinct peak in the intensity of scattered neutrons at sufficiently low temperatures for energies corresponding to this lowest boundary. At the special wave number $q_{p}=\pi(1-2 \sigma)$, this peak should shift down to zero frequency. Our finite-chain matrix elements also indicate that the spectral weight distribution in $S_{z z}(q, \omega)$ has a second discontinuity at $\epsilon_{2 U}(q)$, which is the upper boundary of SWC 2 and the lower bound of excitations with considerably smaller spectral weight. The observability of such a discontinuity may be enhanced by the fact that the density of states at $\epsilon_{2 U}(q)$ is considerably larger than at $\epsilon_{2 L}(q)$. This gives some indication to expect some additional structure at $\epsilon_{2 U}(q)$ in the inelastic neutron scattering cross section. Experimental resolution problems may restrict the observation of the two discontinuities in $S_{z z}(q, \omega)$ to wave numbers in the vicinity of the zero-frequency mode $q_{p}$, and the location of $q_{p}$ depends, of course, on the magnitude of $h$. Figure 19(a) shows the two branches which are expected to be of experimental relevance as solid lines.

Another possible experimental variable is the energy separation of the two discontinuities at $\epsilon_{2 L}(q)$ and $\epsilon_{2 U}(q)$. This (and other possible experimentally interesting quantities) may be obtained from the approximate analytic formulas of Sec. IIC. By using Eqs. (2.21) and (2.22) for the expected discontinuity positions we obtain, for a wave vector fixed in the range $2 \pi \sigma(h)<q<\pi[1-2 \sigma(h)]$, an energy splitting which is proportional to the field

$$
\Delta \epsilon=2 h \sin ^{2} \frac{q}{2}
$$
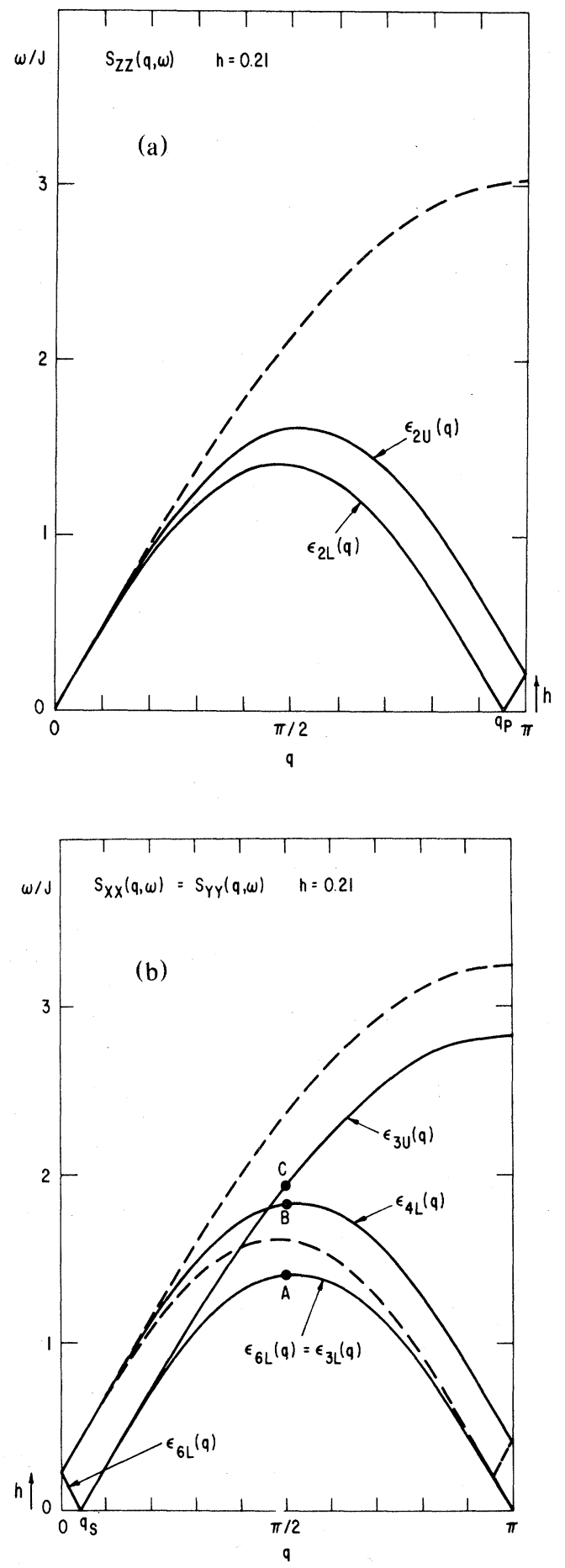

FIG. 19. SWC boundaries of the excitations appearing (a) in the longitudinal and (b) in the transverse structure function at the reduced field $h=0.21$ corresponding to $70 \mathrm{kOe}$ in CPC. The branches drawn as solid lines are predicted by our theory to give rise to observable structures in the inelastic neutron scattering cross section for $h \neq 0$ and at low $T$. The positions $A, B, C$ in (b) correspond to the special energies indicated by vertical lines in the scans of Fig. 20. 
This relation will break down, of course, for $q>\pi(1-2 \sigma)$, and for $q \leqslant 2 \pi \sigma$ we expect only intensity in a small energy range. Recent neutron scattering experiments on the $s=\frac{5}{2}$ linear antiferromagnet TMMC, hitherto regarded as an ideal classical system, in fields up to $70 \mathrm{kOe}$ have revealed ${ }^{47}$ spectra $^{\text {sem }}$ with two peaks, the separation of which is roughly proportional to $h$, which are not explainable in a simple classical spin-wave picture. ${ }^{64}$ Our studies here on the extreme quantum system suggest an explanation of this feature observed in TMMC, in terms of residual quantum effects.

A further potential experimental variable is the energy gap at $q \simeq \pi$ which opens in $S_{z z}(q, \omega)$ for $h \neq 0$. It is proportional to $h$ as is calculated in Eq. (B8) and illustrated in Fig. 15. Note that such features are specific to $S_{z z}(q, \omega)$ for a single crystal. Hence, powder or polycrystalline samples are not suitable for studying these quantum spin dynamical effects at $h \neq 0$, and the orientation of the crystal must be carefully checked.

The transverse correlations have been studied in Sec. IIF. There, we have found that in SWC 3, which dominates $S_{-+}(q, \omega)$ at $T=0$, the spectral weight increases strongly towards the lower boundary $\epsilon_{3 L}(q)$ [see Fig. 17(a)]. We expect therefore that (for low $T$ ) at this energy a clear intensity peak of inelastically scattered neutrons should be observed. At the upper boundary of $\epsilon_{3 U}(q)$, of SWC 3, the finite-chain matrix elements are much smaller. However, if we remember that the density of states in SWC 3 diverges at $\epsilon_{3 U}(q)$ (as it does for $h=0$ ), we can assume that the spectral weight distribution in $S_{-+}(q, \omega)$ of a macroscopic system has a second discontinuity at $\epsilon_{3 U}(q)$, which may also give rise to some structure in the neutron scattering cross section. $S_{+-}(q, \omega)$, on the other hand, has a spectral weight distribution which is considerably more complex. Nevertheless, we have concluded in Sec. II F that for the macroscopic system most of the spectral weight will be concentrated within the two boundaries $\epsilon_{6 L}(q)$ and $\epsilon_{5 U}(q)$. This may again lead at both boundaries to discontinuities in the spectral weight distribution of $S_{+_{-}}(q, \omega)$, which should be observable in the neutron scattering cross section. Note that part of $\epsilon_{6 L}(q)$ coincides with $\epsilon_{3 L}(q)$. It will therefore even enhance the intensity peak expected at this lowest SWC boundary. Figure 19(b) shows those branches in $S_{x x}(q, \omega)$ which are expected to be experimentally observable as solid lines.

A striking difference between $S_{x x}(q, \omega)$ and $S_{z z}(q, \omega)$ is in the behavior at $q=0$. For $S_{x x}(q, \omega)$ there is no weight associated with the ground state, i.e., no static contribution. All the spectral weight at $q=0$ is associated with a state situated at $\omega / J=h$ above the ground state, belonging to the same multiplet. Hence, the experimental scattering intensity at very low temperatures should consist of a narrow peak whose location is proportional to the field and whose magnitude increases as $h \rightarrow h_{c}$. This feature should be easy to detect experimentally.

A further striking difference is the existence of a "soft mode" at $q_{p}=\pi(1-2 \sigma)$ in $S_{z z}(q, \omega)$ and at $q_{s}=2 \pi \sigma$ in $S_{x x}(q, \omega)$. Note that for $h \leqslant \frac{1}{2} h_{c}$ the longitudinal soft mode is much more intense than the transverse. The behavior of these two soft modes may be investigated separately by using a carefully oriented single crystal. Presumably, if the crystal is misoriented, or if a microcrystalline sample is used, intensity at $\omega=0$ should appear both at $q_{s}$ and $q_{p}$ (for $h \neq 0$ ).

In Fig. 20, we compare our theory with low $T$ inelastic neutron scattering measurements on CPC at zero field, 35 and $70 \mathrm{kOe}^{43,65}$ It appears likely, from the account in Ref. 43, that the crystal orientation with respect to the field is such that the data represent the transverse correlations $S_{x x}(q, \omega)$. The vertical lines denoted $A, B$, and $C$ correspond to the energies of the special branches $\epsilon_{3 L}(q)=\epsilon_{6 L}(q)$,

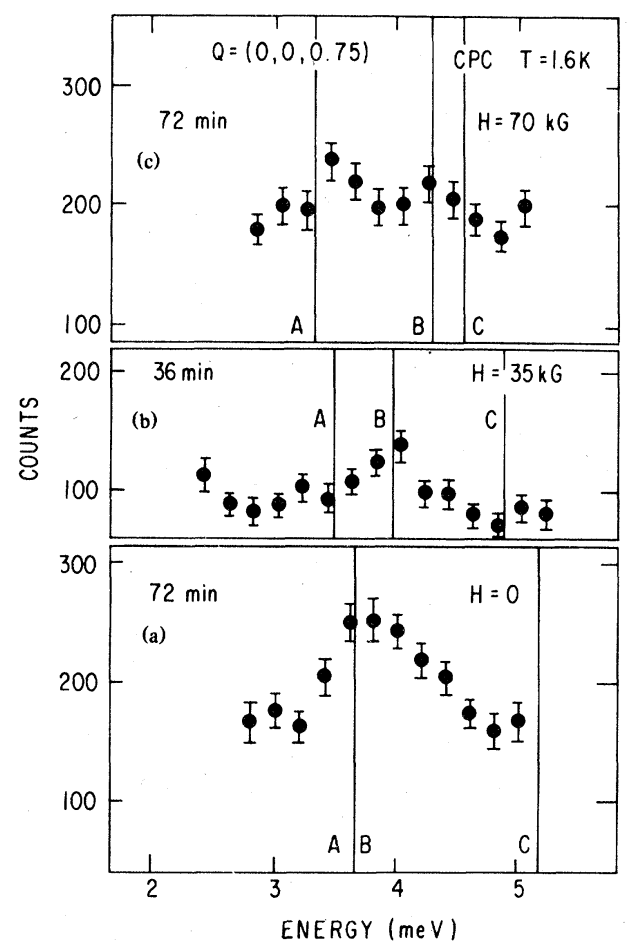

FIG. 20. (a)-(c) Comparison of our theoretical predictions for the peak positions in $S_{x x}(q, \omega)$ with corresponding experimental data for CPC by Heilmann et al. (see Ref. 43). The three scans at $q=\pi / 2$ are for reduced fields $h=0$, 0.105 , and 0.21 , respectively. The vertical lines $A, B, C$ represent the special energies $\epsilon_{3 L}(\pi / 2)=\epsilon_{6 L}(\pi / 2)$, $\epsilon_{4 L}(\pi / 2)$, and $\epsilon_{3 U}(\pi / 2)$, respectively. For $h=0.21$ they are denoted by the same letters as in Fig. 19. 
$\epsilon_{4 L}(q)$, and $\epsilon_{3 U}(q)$, where our theory predicts discontinuities in the $T=0$ transverse structure function [see Fig. 19(b)]. At $h=0$, the maximum intensity is expected to be close to the $\mathrm{dCP}$ energy of 3.63 meV shown as coinciding lines $A, B$ in Fig. 20 (a). This is clearly observed in the experiment, whereas the line $C$ at $5.15 \mathrm{meV}$ is just outside the range of measurements. Figure 20 (c) shows the results for $H=70 \mathrm{kOe}$ corresponding to the reduced field $h=0.21$ used in Fig. 19. Here the experimental data reveal two peaks, which can both be well understood in the framework of our theory. The peak at lower energy again corresponds to the dCP energy $\epsilon_{3 L}(q)$ $=\epsilon_{6 L}(q)$ indicated by line $A$ at $3.2 \mathrm{meV}$ and the peak at higher energy is clearly due to the combined effect of the special energies $\epsilon_{4 L}(q)$ and $\epsilon_{3 U}(q)$, lying close together at $h=0.21$. They are indicated as lines $B$ and $C$ at 4.2 and $4.5 \mathrm{meV}$, respectively. In Fig. 20(b), comparison is made with the data at $35 \mathrm{kOe}$, yielding fair agreement with our theory. At this field the lines $B$ and $C$ are more separated and the data indicate in addition to the peaks corresponding to lines $A(3.45 \mathrm{meV})$ and $B$ ( $3.9 \mathrm{meV}$ ) a third additional, weak signal, corresponding to the line $C$ at $4.8 \mathrm{meV}$. Note, however, that for this field the statistics are poorer because of the shorter counting times. The agreement between our theory and these preliminary data suggest that it would be well worthwhile to repeat the experiment with a larger crystal carefully oriented.

In Fig. 21 we show the integrated intensity $I_{z z}(q)$ for $T=0$ and various values of $h$. The $h=0 \mathrm{in}$ tegrated intensity has already been discussed in Sec. IE. It has been shown (see Fig. 6) that results for $N=10$ are a very good approximation to our analytic expression (1.50) for the infinite system, except for $q=\pi$, where the finite-chain result does not reproduce the logarithmic singularity. Accordingly, the curves in Fig. 21 are for $N=10$. The striking feature of the figure is that it show the relative importance of the various modes. For $h=0$, the $q=\pi$ mode is dominant giving rise to a weak divergence in the integrated intensity (1.50) and a divergence in the corresponding (staggered) susceptibility. As the field increases, the dominant mode corresponds to $q=q_{p}=\pi(1-2 \sigma)$, and the intensity remains almost constant for $q_{p}<q \leqslant \pi$. However, the singularity at $q_{p}$ is apparently much weaker (a finite cusp) than the singularity at $q=\pi$ for $h=0$. Under the assumption that the derivatives at the cusp remain finite for $N \rightarrow \infty$, one obtains a contribution to the correlation function of the form

$$
\begin{aligned}
& \left\langle s_{i}^{\tilde{s}} \tilde{I}+R\right\rangle \sim \cos \left(q_{p} R\right) / R^{2}, \\
& q_{p}=\pi(1-2 \sigma) .
\end{aligned}
$$

Note also that as $h$ increases, the maximum value attained by the integrated intensity falls, and vanishes

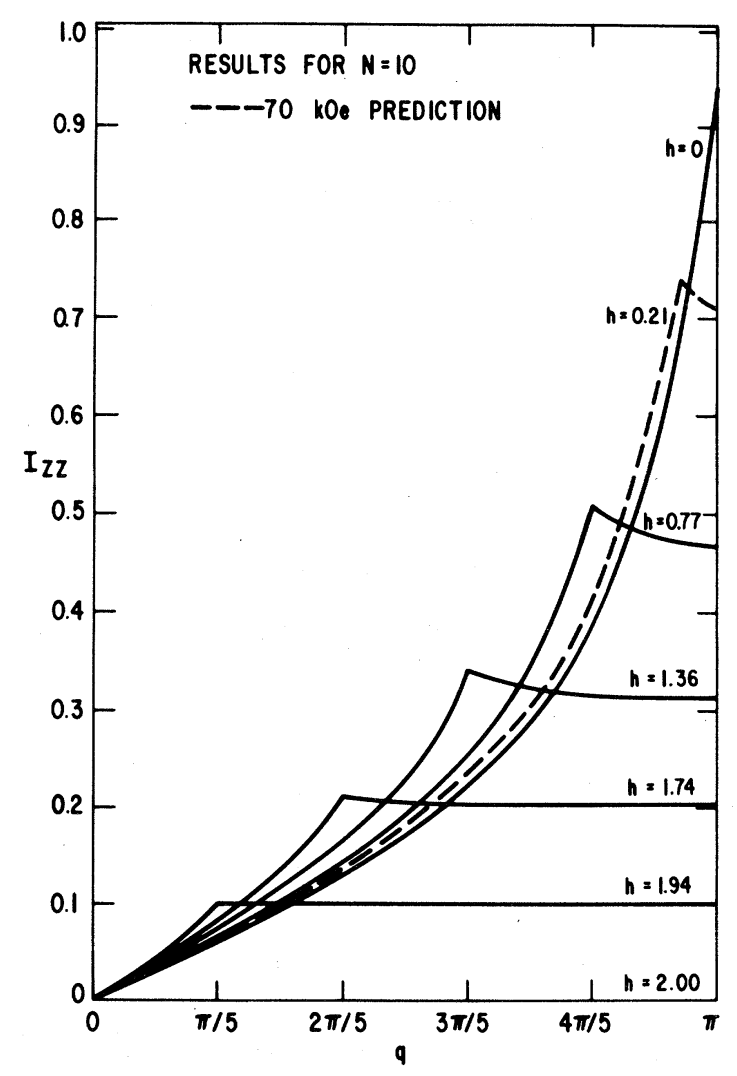

FIG. 21. Finite-chain results $(N=10)$ for the $T=0$ longitudinal integrated intensity at various reduced fields (solid lines). The dashed line is an interpolation for the special field $h=0.21$ corresponding to $H=70 \mathrm{kOe}$ in CPC.

in the limit $h \rightarrow h_{c}$. On the other hand, the sum rule (1.44) tells us that the total intensity is a constant independent of the field. The remainder of the intensity, not shown in Fig. 21, appears as a $\delta$ function at $\omega=0$ and $q=0$ and grows as the field increases. It is indicated in Fig. 15 as the squared diagonal matrix element

$$
M_{G}^{z}=2 \pi \mid\left\langle G\left|s^{z}(0)\right| G \mid\right\rangle^{2}=2 \pi \sigma^{2},
$$

i.e., as the spectral weight of the (field-dependent) ground state itself. It is therefore a static quantity and is omitted from Fig. 21. Of course, it shows up as magnetic Bragg peak in elastic neutron scattering. A further interesting comment concerns the singularity. The fact that the logarithmic divergence at $h=0$ seems to transform into a finite cusp at $h \neq 0$ indicates that the exponent $\frac{1}{2}$ in the expression (1.16) for $S_{z z}(q, \omega)$ at $h=0$ becomes smaller in a (so far unknown) expression at $h \neq 0$ no longer leading to a divergence of the integrated intensity near the zerofrequency mode $q_{p}$. This interesting feature needs further investigation.

Finally, in Fig. 21, the dashed curve is the estimated intensity curve for CPC (with a critical field 
$\left.H_{c}=330 \mathrm{kOe}\right)$ in a field of $70 \mathrm{kOe}$, which corresponds to a reduced field $h=0.21$. The cusp in the curve for $q=0.86 \pi$ and the constancy for larger $q$, i.e., the "truncation of the $h=0$ divergence" (in comparison with Fig. 6) should be experimentally detectable.

We have also calculated the transverse component $I_{x x}(q)$ of the integrated intensity for $T=0$ and various $h$, as shown in Fig. 22. As for $I_{z z}$, convergence is sufficiently rapid that curves for $N=10$ represent the limiting $N=\infty$ behavior very well except at $q=\pi$, where the finite-chain result does not reproduce the logarithmic singularity of Eq. (1.50) at $h=0$. However, it is not clear whether this divergence persists for $h \neq 0$. By comparing $I_{x x}(q)$ with $I_{z z}(q)$ of Fig. 21 we observe a striking difference of the relative importance of the various modes. Whereas the dominant mode $q=q_{p}=\pi(1-2 \sigma)$ in $I_{z z}(q)$ moves with increasing field away from the zone boundary, the dominant mode in $I_{x x}(q)$ remains situated at $q=\pi$ independent of field. However, its weight decreases as $h$ becomes larger and at the critical field $I_{x x}(q)$ is constant as it must be for a system with spins aligned parallel to the $z$ axis. An exact calculation of $I_{x x}(q)$ is possible in the limit $h \rightarrow h_{c}^{-}$for a field range of

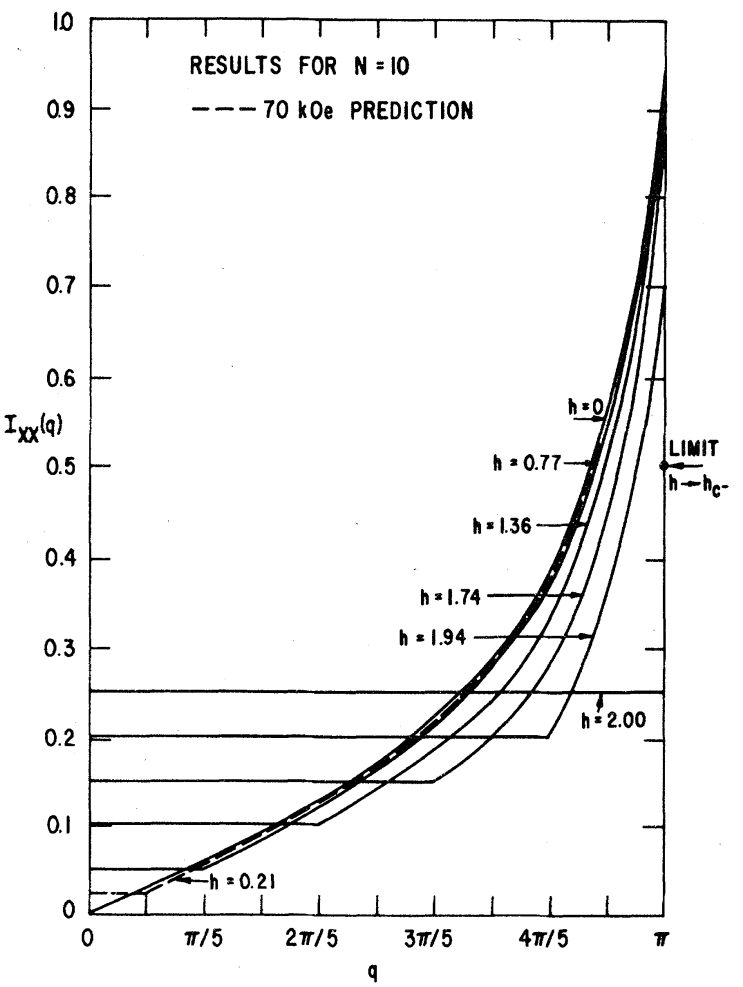

FIG. 22. Finite-chain results $(N=10)$ for the $T=0$ transverse integrated intensity at various reduced fields (solid lines). The dashed line is an interpolation for the special field $h=0.21$ corresponding to $H=70 \mathrm{kOe}$ in CPC. order $h_{c}-h=O\left(N^{-2}\right)$. It yields

$$
I_{x x}(q)=\frac{1}{4}-\frac{1}{2 N}+\frac{1}{4} \delta_{q, \pi}
$$

A characteristic feature of $I_{x x}(q)$ (found empirically) is that in a range of wave numbers increasing with $h$ it is independent of $q$ :

$$
I_{x x}(q)=\frac{1}{2} \sigma, \quad 0 \leqslant q \leqslant 2 \pi \sigma .
$$

Only $I_{x x}(0)=\frac{1}{2} \sigma$ has been derived exactly. For a reduced field of $h=0.21$, corresponding to current neutron scattering studies on CPC in a field of 70 $\mathrm{kOe}$, the estimated curve is shown dashed.

\section{CONCLUDING DISCUSSION AND SUMMARY}

We have presented a new approach to the spin dynamics of quantum linear chains which is quite different from the many-body techniques usually employed. A complete analytical treatment of the 1D Heisenberg dynamics appears out of reach at present, since even the much simpler static problem is not yet analytically solvable for the HB AF. Nevertheless, we have been able to obtain many new analytic results, some of which are exact and some approximate. The deviations from the corresponding (unknown) exact results, as estimated by the application of exact sum rules, are small and well within present experimental accuracy.

Our results have been obtained on the basis of four main techniques: (i) Analytic calculations of a special class of Bethe ansatz states, (ii) exact finite-chain calculations for chains of 4 to 10 spins, (iii) derivation and application of two different types of selection rules, and (iv) the use of various kinds of exact sum rules. Major emphasis has been put on zerotemperature studies in zero and nonzero magnetic field. For zero field we have obtained an approximate analytic expression for the dynamical spin correlation function which fits excellently into the fragmentary picture of the few available exact results. This expression has then been analyzed and exploited to yield a variety of further results of theoretical and experimental interest. Finite-chain calculations have been used to extend our dynamical study to finite temperatures. Our approach can be generalized to several related problems, including (a) the $\mathrm{HB} X Y$ (HB easy plane) antiferromagnet, (b) the HB- Ising (HB easy axis) antiferromagnet, (c) alternating (dimerized) antiferromagnets, and (d) magnetic chains with $s>\frac{1}{2}$. For nonzero field, we are faced with greater complexity. Again, a great deal of information has been obtained on the energies, densities of states, and matrix elements of those excitations which are relevant for the $T=0$ dynamics. However, 
we have so far not been able to condense this information into an analytic expression for the $T=0$ structure function.

Features of particular interest in our work are the following: (i) The excitations which dominate the $T=0$ dynamics are distributed within two-parameter continua rather than along single dispersion branches; (ii) the density of states and the matrix elements of those excitations which govern the dynamics at $T=0$ and $h=0$ are such that they give rise to an asymmetric shape of the structure function; (iii) for $h \neq 0$ there are striking differences between the longitudinal and the transverse correlations, both involving different continua of excitations; (iv) soft modes, whose positions in $q$ are field dependent, appear in both $S_{z z}(q, \omega)$ and $S_{x x}(q, \omega)$, but in different regions of the Brillouin zone; (v) with increasing field, the longitudinal fluctuations are more and more suppressed, whereas the transverse fluctuations become more important. In all these aspects we observe considerable qualitative deviations from the predictions of standard spin-wave theory, which turns out not to be applicable to this problem.

The reason that our calculations are not exact is that they neglect a class of states which are not describable by the special Bethe ansatz prescription (SWC) used in this paper. Their spectral weight is fairly small, but nonvanishing in the thermodynamic limit. Some interesting information is available on states of this type near the critical field, which calls for further investigation into their nature for all fields.

This work was inspired by recent neutron scattering studies on the quasi-1D antiferromagnets CPC and TMMC. Our theory explains existing experimental observations on CPC both qualitatively and quantitatively. Specifically, it explains an asymmetry in the zero-field scattering line shape, it agrees quantitatively with the low-temperature integrated intensity (unlike a semiclassical theory), and it predicts the existence and location of multiple structures in the scattering intensity for $h \neq 0$. Our theory goes on to describe additional effects susceptible to future experimental investigation, such as differences between $S_{z z}(q, \omega)$ and $S_{x x}(q, \omega), I_{z z}(q)$ and $I_{x x}(q)$ at $h \neq 0$, various features of $S_{z z}$ and $S_{x x}$ which are simply related to the magnetic field, e.g., soft-mode locations and energy relations between scattering peaks, and other prominent features of the dynamical correlation functions.

\section{ACKNOWLEDGMENTS}

We are happy to thank Ian Heilman for several discussions and communications, Gen Shirane, John Axe, and Marcos Puga for stimulating discussions, and Pierre Hohenberg for clarifying a problem concerning sum rules. This work was supported by the Swiss National Foundation, and, in part, by NATO and by the U.S. National Science Foundation, Grant No. DMR 80-10819. J.C.B. expresses gratitude to the Institut für Physik of the University of Basel for its hospitality. One of us (J.C.B.) would like to acknowledge a fellowship from the Bunting Institute, Radcliffe College, Cambridge, Mass. 02138.

\section{APPENDIX A: GROUND-STATE ENERGY AND SWC EXCITATION ENERGIES IN HULTHEN'S NOTATION}

Here we evaluate the energies of the eigenstates in the limit of a very large system $(N \rightarrow \infty)$. For this purpose it is necessary to replace the discrete indices $j$ in Bethe's equations (1.20) to (1.22) by a continuous variable $x$. Hulthén ${ }^{25}$ introduced such a transformation which he used to calculate the ground-state energy for $h=0$. Several people, ${ }^{4.7 .24 .59}$ applied this procedure to the calculation of other quantities.

Here, we use the same notation in order to calculate exact expressions for the energies of the SWC states at arbitrary $h$. In Appendix B we present an approximate analytic evaluation of these expressions. It is exact in the two limits $h=0$ and $h=h_{c}=2$.

\section{Ground state in an applied field}

In the limit $N \rightarrow \infty$, Eqs. (1.20) and (1.22) for the ground state in an applied field are transformed into ${ }^{24}$

$$
\begin{aligned}
& 2 \cot \frac{1}{2} \psi(x, y)=\cot \frac{1}{2} k(x)-\cot \frac{1}{2} k(y), \\
& k(x)=2 \pi x+\frac{1}{2} \int_{\sigma}^{1-\sigma} d y \psi(x, y), \\
& E_{G}=\frac{1}{2} N \int_{\sigma}^{1-\sigma} d x[\cos k(x)-1]+E_{F},
\end{aligned}
$$

where the continuous variable $x$ replaces the index $j$. The functions $k(x)$ and $\psi(x, y)$ are defined in the range $0 \leqslant x, y \leqslant 1$, but in order to calculate $E_{G}$ it is sufficient to know them in the range $\sigma \leqslant x, y$ $\leqslant 1-\sigma$. By eliminating $\psi$ in Eq. (A2), differentiating the result with respect to $k$, and substituting

$$
\xi=\cot \frac{1}{2} k(x), \quad f_{G}(\xi)=-\frac{d x}{d \xi},
$$

the coupled Eqs. (A1) and (A2) transform into the linear integral equation

$$
f_{G}(\xi)=g_{G}(\xi)-\int_{-\alpha}^{+\alpha} d \eta K(\xi-\eta) f_{G}(\eta),
$$

with

$$
g_{G}(\xi)=\frac{2}{\pi} \frac{1}{1+\xi^{2}}, \quad K(\xi-\eta)=\frac{2}{\pi} \frac{1}{4+(\xi-\eta)^{2}},
$$


and where $\alpha$ is related to $\sigma$ through

$$
\frac{1}{2}-\sigma=\frac{1}{2} \int_{-\alpha}^{+\alpha} d \xi f_{G}(\xi) \text {. }
$$

Once the solution $f_{G}(\xi)$ of Eq. (A5) is found it is straightforward to calculate the ground-state energy

$$
E_{G}(\sigma)=-\frac{N}{2} \pi \int_{-\alpha}^{+\alpha} d \xi g_{G}(\xi) f_{G}(\xi)+E_{F}
$$

and all related thermodynamic properties. The integral equation (A5) is readily solved for two special cases:

(i) at zero field $(\sigma=0, \alpha=\infty)$ it is solved by Fourier transform, yielding

$$
f_{G}(\xi)=\frac{1}{2} \operatorname{sech} \frac{\pi}{2} \xi
$$

(ii) at the critical field ( $\sigma=\frac{1}{2}, \alpha=0$ ) its solution is trivially given by

$$
f_{G}(\xi)=g_{G}(\xi)
$$

The ground-state energy in these two limits takes the values

$$
E_{G}(0)=-N\left(\ln 2-\frac{1}{4}\right), \quad E_{G}\left(\frac{1}{2}\right)=E_{F}=\frac{1}{4} N .
$$

For intermediate fields $\left(0<\sigma<\frac{1}{2}\right) \mathrm{Eq}$. (A5) has been solved numerically. ${ }^{24,59}$ In Appendix B we present an approximate analytic solution.

\section{Spin-wave continuum 1}

The states of the SWC 1 are characterized by sets of $\lambda_{j}$ obeying the rules (2.11). In order to account for the gaps between the $\lambda_{j}$ clusters we have to alter Eqs. (A2) and (A3) as follows

$$
\begin{aligned}
k(x)= & 2 \pi x+\frac{1}{2} \int_{\sigma}^{1-\sigma} d y \psi(x, y)-\frac{1}{N} \psi\left(x, x_{1}\right) \\
& -\frac{1}{N} \psi\left(x, x_{2}\right) \\
E_{1, m}(q)= & E_{F}+\frac{1}{2} N \int_{\sigma}^{1-\sigma} d x[\cos k(x)-1] \\
& -\left[\cos k\left(x_{1}\right)-1\right] \\
& -\left[\cos k\left(x_{2}\right)-1\right] .
\end{aligned}
$$

The gap positions $x_{1}, x_{2}$, are related to the quantum numbers $q, q_{m}, q_{s}$ of the SWC 1 excitations through

$$
\begin{aligned}
& x_{1}=\left(q_{s}+q_{m}\right) / 2 \pi, \\
& x_{2}=\left(2 q-q_{s}-q_{m}\right) / 2 \pi,
\end{aligned}
$$

with

$$
\begin{aligned}
& q_{s}=2 \pi \sigma, \quad q_{s} \leqslant q \leqslant \pi, \\
& 0 \leqslant q_{m} \leqslant q-q_{s} .
\end{aligned}
$$

The same transformations as above with $f_{1}$ replacing $f_{G}$ in Eq. (A4) lead for these states to

$$
f_{1}(\xi)=g_{1}(\xi)-\int_{-\alpha}^{+\alpha} d \eta K(\xi-\eta) f_{1}(\eta),
$$

with

$$
\begin{aligned}
\Delta g(\xi) & \equiv g_{1}(\xi)-g_{G}(\xi) \\
& =\frac{4}{\pi N} \frac{1}{4+\left(\xi-\xi_{1}\right)^{2}}+\frac{4}{\pi N} \frac{1}{4+\left(\xi-\xi_{2}\right)^{2}}
\end{aligned}
$$

and

$$
\begin{aligned}
\omega_{1 m}(q) \equiv & E_{1 m}(q)-E_{G} \\
= & -\frac{N}{2} \pi \int_{-\alpha}^{+\alpha} d \xi g_{G}(\xi)\left[f_{1}(\xi)-f_{G}(\xi)\right] \\
& +\pi\left[g_{G}\left(\xi_{1}\right)+g_{G}\left(\xi_{2}\right)\right] .
\end{aligned}
$$

Equation (A16) can be formally solved by the resolvent $R$

$$
f_{1}(\xi)=g_{1}(\xi)-\int_{-\alpha}^{+\alpha} d \eta R(\xi-\eta) g_{1}(\eta),
$$

which depends on the integral kernel $K$ according to $R(\xi-\zeta)=K(\xi-\zeta)-\int_{\alpha}^{\alpha} d \eta K(\xi-\eta) R(\eta-\zeta)$.

If we set for the solution $f_{1}(\xi)=f_{G}(\xi)+\Delta f_{1}(\xi)$, Eq. (A19) yields

$$
\Delta f_{1}(\xi)=\frac{2}{N} R\left(\xi-\xi_{1}\right)+\frac{2}{N} R\left(\xi-\xi_{2}\right)
$$

and for the excitation energies we obtain

$$
\omega_{1 m}(q)=\pi\left[f_{G}\left(\xi_{1}\right)+f_{G}\left(\xi_{2}\right)\right] .
$$

The relation between $\xi_{1}, \xi_{2}$ and the quantum numbers $q, q_{m}$ determined by $x_{1}, x_{2}$ through Eq. (A14) is given according to Eq. (A4) by

$$
\begin{aligned}
& x=-\int_{0}^{\xi} d \xi^{\prime} f_{G}(\xi)+\text { const }, \\
& \sigma \leqslant x \leqslant 1-\sigma,
\end{aligned}
$$

where terms $O\left(N^{-1}\right)$ are neglected. Thus, we have reduced the SWC 1 excitation problem to the solution of the ground-state equation (A5). An approximate result for Eq. (A22) is presented in Appendix B.

\section{Spin-wave continuum 2}

The states of the SWC 2 are characterized by sets of $\lambda_{j}$ obeying the rules (2.13) and (2.14). In order to account for the gap between the two $\lambda_{j}$ clusters and for the isolated $\lambda_{0}$ we have to take in the continuum limit the following prescription replacing Eqs. (A2) and (A3) 


$$
\begin{aligned}
& k(x)=2 \pi x+\frac{1}{2} \int_{\sigma}^{1-\sigma} d y \psi(x, y)-\frac{1}{N} \psi\left(x, x_{i}\right)+\frac{1}{N} \psi\left(x, x_{0}\right), \quad \sigma \leqslant x \leqslant 1-\sigma, \\
& k\left(x_{0}\right)=2 \pi x_{0}+\frac{1}{2} \int_{\sigma}^{1-\sigma} d y \psi\left(x_{0}, y\right)-\frac{1}{N} \psi\left(x_{0}, x_{1}\right), \quad 0 \leqslant x_{0}, \leqslant \sigma \\
& E_{2 m}(q)=E_{F}+\frac{1}{2} N \int_{\sigma}^{1-\sigma} d x[\cos k(x)-1]+\left[\cos k\left(x_{0}\right)-1\right]-\left[\cos k\left(x_{1}\right)-1\right] .
\end{aligned}
$$

The "particle position" $x_{0}$ and the "hole position" $x_{1}$ are related to the quantum numbers $q, q_{s}, q_{m}$ of the SWC 2 excitations through

$$
\begin{aligned}
& x_{0}=\left(q_{s}-q_{m}\right) / 2 \pi, \\
& x_{1}=\left(2 q+q_{s}-2 q_{m}\right) / 2 \pi
\end{aligned}
$$

with

$$
\begin{aligned}
& q_{s}=2 \pi \sigma, \quad 0 \leqslant q_{m} \leqslant q_{s}, \\
& q_{m} \leqslant q \leqslant \pi-q_{s}+q_{m} .
\end{aligned}
$$

Equation (A24) with Eq. (A4) transforms into an integral equation of the same type as Eq. (A16)

$$
f_{2}(\xi)=g_{2}(\xi)-\int_{-\alpha}^{+\alpha} d \eta K(\xi-\eta) f_{2}(\eta) .
$$

But with a different inhomogeneity term

$$
\begin{aligned}
\Delta g_{2}(\xi) & \equiv g_{2}(\xi)-g_{G}(\xi) \\
& =\frac{4}{\pi N} \frac{1}{4+\left(\xi-\xi_{1}\right)^{2}}-\frac{4}{\pi N} \frac{1}{4+\left(\xi-\xi_{0}\right)^{2}},
\end{aligned}
$$

Eq. (A25) with Eq. (A4) transforms into an integral expression for $f_{2}\left(\xi_{0}\right)$

$$
\begin{aligned}
f_{2}\left(\xi_{0}\right)= & \frac{1}{2} g_{G}\left(\xi_{0}\right)-\frac{1}{2} \int_{-\alpha}^{+\alpha} d \eta K\left(\xi_{0}-\eta\right) f_{2}(\eta) \\
& +O\left(N^{-1}\right) .
\end{aligned}
$$

The factors $\left(\frac{1}{2}\right)$ arising in Eq. (A31) are due to the fact that $x_{0}$ lies outside the range of integration in Eq. (A25) which yields a different derivative with respect to $x$. As is readily seen below, terms $O\left(N^{-1}\right)$ in Eq. (A31) can be neglected. Finally, Eq. (A26) for the SWC 2 excitation energies transforms into

$$
\begin{aligned}
\omega_{2 m}(q) \equiv & E_{2 m}(q)-E_{G} \\
= & -\frac{N}{2} \pi \int_{-\alpha}^{+\alpha} d \xi g_{G}(\xi)\left[f_{2}(\xi)-f_{G}(\xi)\right] \\
& +\pi\left[g_{G}\left(\xi_{1}\right)-g_{G}\left(\xi_{0}\right)\right] .
\end{aligned}
$$

In analogy to Eqs. (A19) and (A20), Eq. (A29) is formally solved by the same reciprocal kernel $R$ so that if we set $f_{2}(\xi)=f_{G}(\xi)+\Delta f_{2}(\xi)$ we obtain

$$
\Delta f_{2}(\xi)=\frac{2}{N} R\left(\xi-\xi_{1}\right)-\frac{2}{N} R\left(\xi-\xi_{0}\right) \text {. }
$$

By using this solution we obtain for the integral (A31)

$$
f_{2}\left(\xi_{0}\right)=\frac{1}{2} f_{G}\left(\xi_{0}\right)+O\left(N^{-1}\right)
$$

With Eq. (A33) the integral in Eq. (A32) can be evaluated and we obtain for the excitation energies of the SWC 2

$$
\omega_{2 m}(q)=\pi\left[f_{G}\left(\xi_{1}\right)-f_{G}\left(\xi_{0}\right)\right] .
$$

According to Eqs. (A4), (A33), and (A34) $\xi_{1}, \xi_{0}$ are related to $x_{1}, x_{0}$ of Eq. (A27) by

$$
\begin{aligned}
x=-\int_{0}^{\xi} d \xi^{\prime} f_{G}\left(\xi^{\prime}\right)+c_{1}, & \\
\sigma & \leqslant x \leqslant 1-\sigma,
\end{aligned}
$$

in accordance with Eq. (A23) and

$$
\begin{aligned}
x=-\frac{1}{2} \int_{0}^{\xi} d \xi^{\prime} f_{G}\left(\xi^{\prime}\right)+c_{2}, & \\
& 0 \leqslant x \leqslant \sigma .
\end{aligned}
$$

Thus, also the calculation of the SWC 2 excitation energies is reduced to the ground-state problem (A5), which is solved approximately in Appendix B.

\section{APPENDIX B: APPROXIMATE SOLUTION OF HULTHEN'S INTEGRAL EQUATION FOR NONZERO FIELD}

In order to evaluate the excitation energies (A22) and (A35) of both continua, SWC 1 and SWC 2, we have to know the solution $f_{G}(\xi)$ of the Hulthén integral equation (A5). Since we know the exact solutions (A9) and (A10) for the two limiting cases $h=0$ and $h_{c}$, respectively, we may approximate the intermediate case by some interpolation formula consisting of a linear combination of Eqs. (A9) and (A10) with coefficients determined by a selfconsistent calculation. Unfortunately the evaluation of excitation energies with such an ansatz leads to complicated implicit equations. It is simpler if we introduce an interpolation formula in the $x$ representation rather than in the $\xi$ representation of Bethe's formalism. In that case it is straightforward to evaluate the excitation energies of both SWC 1 and SWC2. However, an entirely self-consistent calculation would lead to a complexity amenable only to numerical work. 
Our starting point is the prescription (A36) which relates the variables $\xi$ and $x$ to each other. We calculate the analog of $f_{G}(\xi)$ in $x$ space

$$
F(x) \equiv-\frac{d x}{d \xi}=f_{G}(\xi(x))
$$

and obtain by transforming Eq. (A9)

$$
F_{1}(x)=\left\{\begin{array}{l}
\frac{1}{2} \sin \pi x, \quad \sigma \leqslant x \leqslant 1-\sigma \\
\frac{1}{2} \sin \left(2 \pi x-q_{s} / 2\right), \quad 0 \leqslant x \leqslant \sigma
\end{array}\right.
$$

and by transforming Eq. (A10)

$$
F_{2}(x)=\left\{\begin{array}{l}
\frac{1}{\pi}(1+\sin \pi x), \quad \sigma \leqslant x \leqslant 1-\sigma \\
\frac{1}{\pi}\left[1+\sin \left(2 \pi x-\frac{q_{s}}{2}\right)\right], \quad 0 \leqslant x \leqslant \sigma .
\end{array}\right.
$$

Here, the constants $c_{1}$ and $c_{2}$ in Eq. (A36) have been fixed such that Eq. (B2) inserted into Eq. (A22) reproduces the exactly known zero-field SWC (1.27) for $q_{s}=0$ and Eq. (B3) inserted into Eq. (A35) yields the exactly known ferromagnetic spin-wave branch ${ }^{50}$ at the critical field, i.e., for $q_{s}=\pi$.

As a simple ansatz for arbitrary fields $0 \leqslant h \leqslant h_{c}$. $=2$ we choose the linear superposition

$$
F_{G}(x)=\left(1-\frac{B}{2}\right) F_{1}(x)+\frac{B}{2} F_{2}(x),
$$

where $B$ is a parameter to be determined below with the limiting values $B=0$ for $\sigma=h=0$ and $B=2$ for $\sigma=\frac{1}{2}, h=h_{c}=2$. If we use our ansatz (B4) in the exact expressions (A22) and (A35) we obtain for the excitation energies of SWC 1

$$
\omega_{1 m}(q)=2 D \sin \frac{q}{2} \cos \left(\frac{q}{2}-\frac{q_{s}}{2}-\frac{q_{m}}{2}\right),
$$

with quantum numbers $q, q_{s}, q_{m}$ in the range (A15) and for SWC 2

$$
\omega_{2 m}(q)=2 D \sin \frac{q}{2} \cos \left(\frac{q}{2}+\frac{q_{s}}{2}-\frac{q_{m}}{2}\right),
$$

with quantum numbers $q, q_{s}, q_{m}$ in the range (A27). In Eq. (B6) a constant term, which is obviously due to the approximate character of our ansatz, has been dropped. The prefactor $D$ is related to the parameter $B$ through

$$
D=\frac{B}{2}\left(1-\frac{\pi}{2}\right)+\frac{\pi}{2} \text {. }
$$

We are left with the problem of determining the parameter $B$ as a function of $h$ or $\sigma$. Since our purpose here is to provide a simple approximation with handy formulas, we avoid at this stage a selfconsistent calculation, which would involve numerical evaluation of complex expressions. An alternative way uses the exact result that the energy of the lowest excitation contributing to $S_{z z}(q, \omega)$ at the zone boundary is proportional to the magnetic field ${ }^{59}$

$$
\omega_{1 m}(\pi)=\omega_{2 m}(\pi)=2 D \sin \pi \sigma=h,
$$

with $q_{m}=0$ and $q_{m^{\prime}}=q_{s}$. This determines the parameter $D$ (or $B$ ) as a function of $\sigma$ and $h$. Unfortunately, there is no exact analytic expression available for the $T=0$ magnetization curve $\sigma(h)$, which would determine our parameters $D$ and $B$ as a function of $\sigma$ or $h$ alone. However, we can compare Eq. (B8) with the magnetization curve which was calculated numerically by Griffiths. ${ }^{24}$ This comparison yields a nearly linear dependence of $D$ on $h$. Therefore, in order to have a simple expression we assume a linear dependence without making our approximation significantly worse; i.e., we identify our parameter $B$ with the magnetic field

$$
B \equiv h \text {. }
$$

This provides us with a simple but effective analytic expression for the $T=0$ magnetization curve

$$
\sigma(h)=\pi^{-1} \arcsin [h / 2 D(h)] .
$$

1P. W. Anderson, Phys. Rev. 86, 694 (1952).

${ }^{2} \mathrm{M}$. T. Hutchings, G. Shirane, R. J. Birgeneau, and S. L. Holt, Phys. Rev. B $\underline{5}, 1999$ (1972).

${ }^{3}$ E. H. Lieb and D. C. Mattis, J. Math. Phys. 3, 749 (1962).

${ }^{4}$ J. des Cloizeaux and J. J. Pearson, Phys. Rev. 128, 2131 (1962).

5 J. C. Bonner, thesis (University of London, 1968) (unpublished), Chap. 3.

${ }^{6} \mathrm{~J}$. C. Bonner, B. Sutherland, and P. M. Richards, in
Proceedings of the 20th Annual Conference on Magnetism and Magnetic Materials-1974 (San Francisco), edited by C. D. Graham, Jr., G. H. Lander, and J. J. Phyne, AIP Conf. Proc. No. 24 (AIP, New York, 1975), p. 335.

${ }^{7}$ T. Yamada, Prog. Theor. Phys. Jpn. 41, 880 (1969).

${ }^{8} \mathrm{G}$. Müller, H. Thomas, and H. Beck, in Ordering in Strongly Fluctuating Condensed Matter Systems, edited by T. Riste (Plenum, New York, 1980), p. 151 (Proceedings of the NATO AS1 Conference, Geilo, Norway, 1979). 
${ }^{9}$ H. A. Bethe, Z. Phys. 71, 205 (1931).

10J. D. Johnson and B. M. McCoy, Phys. Rev. A $\underline{6}, 1613$ (1972).

11P. Fazekas and P. Sütö, Solid State Commun. 19, 1045 (1976).

12P. C. Hohenberg and W. F. Brinkman, Phys. Rev. B $\underline{10}$, 128 (1974).

${ }^{13} \mathrm{Th}$. Niemeijer, Physica (Utrecht) $\underline{36}, 377$ (1967).

${ }^{14}$ R. K. G. Liem, thesis (Carnegie-Mellon University, 1969) (unpublished).

${ }^{15}$ S. Katsura, T. Horiguchi, and M. Suzuki, Physica (Utrecht) 46, 67 (1970).

${ }^{16}$ Y. Endoh, G. Shirane, R. J. Birgeneau, P. M. Richards, and S. L. Holt, Phys. Rev. Lett. 32, 170 (1974).

${ }^{17}$ T. Todani and K. Kawasaki, Prog. Theor. Phys. Jpn. $\underline{50}$, 1216 (1973)

${ }^{18}$ L. N. Bulaevskii, Sov. Phys. JETP $\underline{16}, 685$ (1963) [Zh. Eksp. Teor. Fiz. 43, 968 (1962)].

${ }^{19}$ H. J. Mikeska, Phys. Rev. B 12, 2794 (1975).

${ }^{20} \mathrm{G}$. Müller, H. Beck, and J. C. Bonner, Phys. Rev. Lett. 43,75 (1979).

${ }^{21}$ G. Müller, H. Beck, and J. C. Bonner, J. Appl. Phys. 50, 7404 (1979).

${ }^{22}$ G. Müller, H. Beck, and J. C. Bonner, J. Magn. Magn. Mater. 15-18, 367 (1980).

${ }^{23}$ P. M. Richards and F. Carboni, Phys. Rev. B $\underline{5}, 2014$ (1972).

${ }^{24}$ R. B. Griffiths, Phys. Rev. 133, A768 (1964).

${ }^{25}$ L. Hulthén, Arkiv Mat. Astron. Fysik 26A, 1 (1938).

${ }^{26}$ J. des Cloizeaux and M. Gaudin, J. Math. Phys. I, 1384 (1966).

${ }^{27}$ G. Müller and H. Beck, J. Phys. C 11 , 483 (1978).

${ }^{28} \mathrm{G}$. Müller et al. (unpublished).

${ }^{29}$ E. H. Lieb, T. Schultz, and D. C. Mattis, Ann. Phys. (N.Y.) 16, 407 (1961).

${ }^{30}$ Note that the Hohenberg-Brinkman $C_{\mu \mu}$ is our $I_{\mu \mu}$ and their $S_{\mu \mu}$ is half of our $S_{\mu \mu}$.

${ }^{31} \mathrm{We}$ are indebted to Professor Hohenberg for a correspondence clarifying this point.

${ }^{32}$ C. N. Yang and C. P. Yang, Phys. Rev. 151, 258 (1966).

${ }^{33}$ I. S. Gradshteyn and I. M. Ryzhik, Tables of Integrals, Series, and Products (Academic, New York, 1965).

34J. C. Bonner and M. E. Fisher, Phys. Rev. 135, A640 (1964).

${ }^{35}$ For the $X Y$ model, for example, all sum rules are consistent with the constant " $A$ " $=2$ in front of Eq (1.35).

${ }^{36}$ C. P. Yang, in Proceedings of the Conference on Quantum Lattice Gas and the Heisenberg-Ising Antiferromagnetic Chain (unpublished); B. Sutherland (private communication).

${ }^{37}$ J. P. Boucher, L. P. Regnault, J. Rossat-Mignod, J. P. Renard, J. Bouillot, and W. G. Stirling, Solid State Commun. $\underline{33}, 171(1980)$.
${ }^{38} \mathrm{R}$ : B. Griffiths (unpublished)

${ }^{39}$ M. E. Fisher, Am. J. Phys. 32, 343 (1964).

${ }^{40}$ M. F. Thorpe, J. Phys. (Paris) 36, 1177 (1975).

${ }^{41}$ A. Luther and I. Peschel, Phys. Rev. B 12, 3908 (1975).

${ }^{42}$ H. C. Fogedby, J. Phys. C 11,4767 (1978).

${ }^{43}$ I. U. Heilmann, G. Shirane, Y. Endoh, R. J. Birgeneau and S. L. Holt, Phys Rev. B 18 , 3530 (1978).

${ }^{44} \mathrm{~T}$. Holstein and H. Primakoff, Phys. Rev. $\underline{58}, 1098$ (1940).

${ }^{45}$ H. J. Mikeska and W. Pesch, J. Phys. C $\underline{12}$, L37 (1979).

${ }^{46}$ The result for $S_{z z}(q, \omega)$ given by Luther and Peschel themselves is obviously wrong.

${ }^{47}$ I. U. Heilmann, R. J. Birgeneau, Y. Endoh, G. Reiter, G. Shirane, and S. L. Holt, Solid State Commun. 31, 607 (1979).

48J. P. Groen, T. O. Klaassen, and N. J. Poulis, Phys. Lett. 68A, 381 (1978)

${ }^{49}$ J. P. Groen, H. W. Capel, J. H. H. Perk, T. O. Klaassen, and N. J. Poulis, Physica (Utrecht) 97B, 126 (1979).

50J. P. Groen, T. O. Klaassen, N. J. Poulis, G. Müller, H. Thomas, and H. Beck, Phys Rev. B 22, 5369 (1980).

${ }^{51}$ L. J. Azevedo, A. Narath, P. M. Richards, and Z. G. Soos, J. Appl. Phys. 50, 1859 (1979).

${ }^{52}$ L. J. Azevedo, A. Narath, P. M. Richards, and Z. G. Soos, Phys. Rev. Lett. 43, 875 (1979).

${ }^{53}$ L. J. Azevedo, A. Narath, P. M. Richards, and Z. G. Soos, Phys. Rev. B 21, 2871 (1980).

${ }^{54} \mathrm{~J}$. W. Bray, H. R. Hart, Jr., L. V. Interrante, I. S. Jacobs, J. S. Kasper, G. D. Watkins, and S. H. Wee, Phys. Rev. Lett. 35,744 (1975).

${ }^{55}$ I. S. Jacobs, J. W. Bray, H. R. Hart, Jr., L. V. Interrante, J. S. Kasper, G. D. Watkins, D. E. Prober, and J. C. Bonner, Phys. Rev. B 14, 3036 (1976).

${ }^{56}$ L. V. Interrante, J. W. Bray, H. R. Hart, Jr., I. S. Jacobs, J. S. Kasper, P. A. Piacente, and J. C. Bonner, in Proceedings of the International Conference on Quasi-OneDimensional Conductors, Dubrovnik, Yugoslavia, 1978, published in Lecture Notes in Physics (Springer-Verlag, New York, 1979), Vol. 56, p. 55.

${ }^{57} \mathrm{~F}$. Keffer, in Handbuch der Physik, edited by S. Flügge (Springer, Berlin, 1966), p. 135

${ }^{58}$ E. Pytte, Phys. Rev. B 10, 4637 (1974).

${ }^{59}$ N. Ishimura and H. Shiba, Prog. Theor. Phys. Jpn. 57, 1862 (1977)

${ }^{60} \mathrm{M}$. W. Puga, Phys. Rev. Lett. 42, 405 (1979).

${ }^{61}$ J. C. Bonner and J. F. Nagle, Phys. Rev. A 5, 2293 (1972).

${ }^{62} \mathrm{G}$. Müller and H. Beck, J. Magn. Magn. Mater. 15-18, 689 (1980)

${ }^{63}$ S. Katsura, Ann. Phys. (N.Y.) 31, 325 (1965).

${ }^{64} \mathrm{G}$. Reiter (private communication).

${ }^{65} \mathrm{We}$ are indebted to $\mathrm{G}$. Shirane for extensive and illuminating discussions on the interpretation and significance of the experimental results. 